\title{
Enhanced Field Emission Studies on Niobium Surfaces Relevant to High Field Superconducting Radio- Frequency Devices
}

by

\section{Tong Wang}

Dissertation submitted to the Faculty of the Virginia Polytechnic Institute and State University in partial fulfillment of the requirements for the degree of

\section{DOCTOR OF PHILOSOPHY}

in

\section{PHYSICS}

C. E. Reece, Co-chairman

J. R. Ficenec, Co-chairman

R. M. Sundelin

D. A. Jenkins

L. W. Mo

J. R. Morris

September 18, 2002

Blacksburg, Virginia

Keywords: electron field emission, enhanced field emission, radio-frequency

superconductivity, niobium

Copyright 2002, Tong Wang 


\title{
Enhanced Field Emission Studies on Niobium Surfaces Relevant to High Field Superconducting Radio- Frequency Devices
}

\author{
by \\ Tong Wang \\ Committee Co-chairmen: Drs. C. E. Reece/J. R. Ficenec \\ Department of Physics
}

\begin{abstract}
Enhanced field emission (EFE) presents the main impediment to higher acceleration gradients in superconducting niobium $(\mathrm{Nb})$ radiofrequency cavities for particle accelerators. The strength, number and sources of EFE sites strongly depend on surface preparation and handling.

The main objective of this thesis project is to systematically investigate the sources of EFE from $\mathrm{Nb}$, to evaluate the best available surface preparation techniques with respect to resulting field emission, and to establish an optimized process to minimize or eliminate EFE.

To achieve these goals, a scanning field emission microscope (SFEM) was designed and built as an extension to an existing commercial scanning electron microscope (SEM). In the SFEM chamber of ultra high vacuum, a sample is moved laterally in a raster pattern under a high voltage anode tip for EFE detection and localization. The sample is then transferred under vacuum to the SEM chamber equipped with an energy-dispersive $\mathrm{x}$-ray spectrometer for individual emitting site characterization. Compared to other systems built for similar purposes, this apparatus has low cost and maintenance, high operational flexibility, considerably bigger scan area, as well as reliable performance.
\end{abstract}


EFE sources from planar $\mathrm{Nb}$ have been studied after various surface preparation, including chemical etching and electropolishing, combined with ultrasonic or highpressure water rinse. Emitters have been identified, analyzed and the preparation process has been examined and improved based on EFE results. As a result, fieldemission-free or near field-emission-free surfaces at $\sim 140 \mathrm{MV} / \mathrm{m}$ have been consistently achieved with the above techniques. Characterization on the remaining emitters leads to the conclusion that no evidence of intrinsic emitters, i.e., no fundamental electric field limit induced by EFE, has been observed up to 140 $\mathrm{MV} / \mathrm{m}$. Chemically etched and electropolished $\mathrm{Nb}$ are compared and no significant difference is observed up to $\sim 140 \mathrm{MV} / \mathrm{m}$.

To address concerns on the effect of natural air drying process on EFE, a comparative study was conducted on $\mathrm{Nb}$ and the results showed insignificant difference under the experimental conditions.

$\mathrm{Nb}$ thin films deposited on $\mathrm{Cu}$ present a possible alternative to bulk $\mathrm{Nb}$ in superconducting cavities. The EFE performance of a preliminary energetically deposited $\mathrm{Nb}$ thin film sample are presented. 


\section{Acknowledgements}

I would like to express my deepest gratitude to Dr. Ronald Sundelin, my former advisor until his retirement in January 2002. Without him offering me the opportunity of studying at VPI\&SU and pursuing my education under his advisement, without his constant guidance along the way, this thesis would not have been possible. I am grateful for the privilege of witnessing and benefiting from the breadth and depth of his knowledge and meticulous work ethic. I also would like to thank Dr. Reece, who has advised me along with Dr. Sundelin since the very beginning and became my advisor after Ron's retirement. His sound technical knowledge and communication skills always bring a new perspective into this work. I am grateful to them for their skillful way of combining guidance, encouragement with trust and freedom that had allowed me to rely on my own judgement in pursuing some of the experiments.

I am indebted to Dr. Larry Phillips for his help during the construction of the experimental apparatus. He has voluntarily offered help in many occasions, and has always been kind, supportive and generous. I am also grateful to Dr. Peter Kneisel for always offering me the latest technical papers, for helping me set up the electropolishing equipment and lending me the mock cavity for some experiments. Sincere thanks goes to Prof. Ficenec for carefully reviewing this thesis.

Assistance from Brett Lewis in leak check and many other occasions, from Tom Goodman for the maintenance of the SEM and EDS, from Ralph Afanador with chemical etching and high pressure water rinse, from Pete Kushnick with some 
computer problems, and from John Brawley for electron-beam welding of some samples are all sincerely appreciated.

I wish to thank Anne-Marie Valente for valuable discussion that led to the experiments on the drying process of Nb, Ricky Campisi and Jean Delayen for the careful review of my paper submitted to Review of Scientific Instruments. Thanks especially to Julie Oyer for preparing all the purchase orders, taking care of all the chores... ... A special thanks goes to Chris Thomas for taking care of everything for me on campus, from all the official forms to personal assistance, when I am away all year long at Newport News.

On a personal level, I'd like to thank my Mom and Dad for exposing me to physics at a young age, for playing a pivotal role in my choice of education, and for always supporting me unconditionally. My brother and sister have always been extremely generous toward me, financially and emotionally, and their support and understanding are always appreciated. I also would like to extend my gratitude to my parents-in-law for all the support and love they've given me. Finally I would like to thank my husband, Xiaodong, for never hesitating in offering his ideas and suggestions on my experiments, for preparing many of the figures in this thesis, and for always being there for me all the years......

* The work was supported by the U.S. DOE Contract No. DE-AC05-84ER40150. 


\section{Table of Contents}

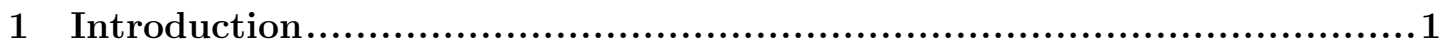

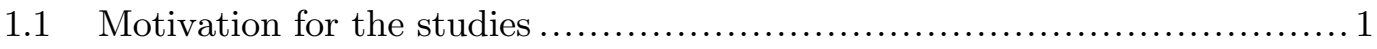

1.1.1 Superconducting RF cavities ................................. 1

1.1.2 High voltage vacuum devices and other applications $\ldots \ldots \ldots \ldots \ldots \ldots 7$

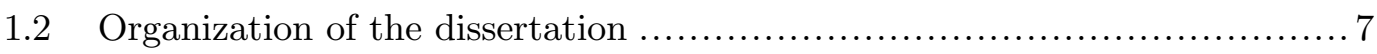

2 Field Emission Fundamentals ............................................... 9

2.1 Field emission theory and enhanced field emission ........................ 9

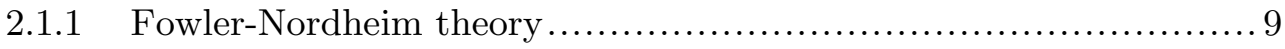

2.1.2 Enhanced field emission....................................... 11

2.2 General characteristics of enhanced field emission........................ 12

2.2.1 Current instability and conditioning effect ...................... 12

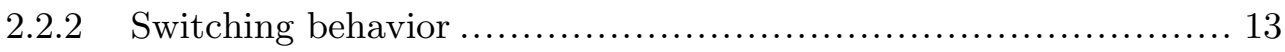

2.2.3 Hysteresis in I-V characteristics ............................. 14

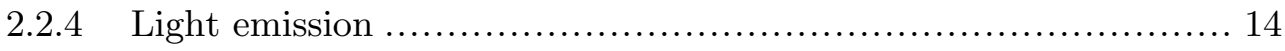

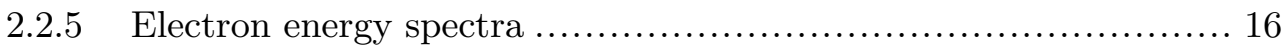

2.2.6 Pre-breakdown and breakdown $\ldots \ldots \ldots \ldots \ldots \ldots \ldots \ldots \ldots \ldots \ldots \ldots \ldots \ldots \ldots$

2.3 Some models proposed for enhanced field emission ....................... 18

2.3.1 Geometrical enhancement model ................................. 18

2.3.2 Metal-insulator-vacuum model............................... 21

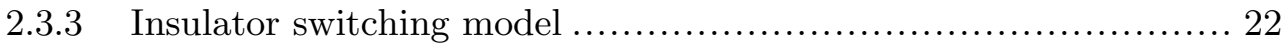

2.3.4 Metal-insulator-metal model................................. 24 
2.3.5 Filament model................................................ 24

2.3.6 Resonant tunneling model ..................................... 25

2.3.7 Thermionic emission model .................................. 25

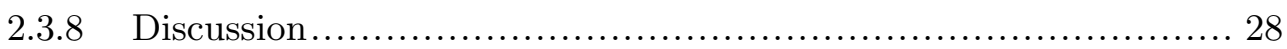

\section{Summary of previous research results on enhanced field emission from}

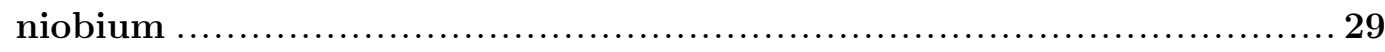

3.1 Results from University of Geneva....................................... 30

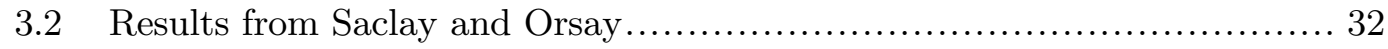

3.3 Results from Cornell University ...................................... 32

3.4 Results from University of Wuppertal ................................. 34

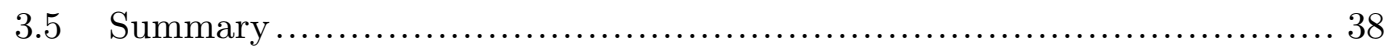

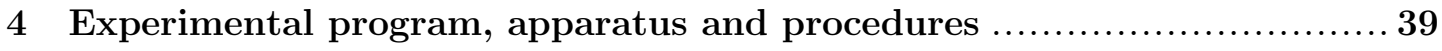

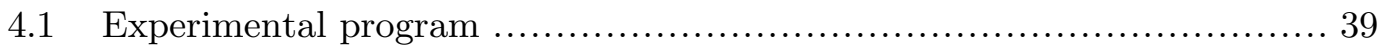

4.1.1 Experimental objectives..................................... 39

4.1.2 Experimental plan ............................................ 42

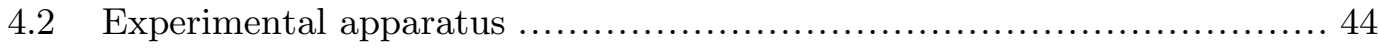

4.2.1 Ultra high vacuum chambers design ............................ 44

4.2.2 Sample and anode manipulation and sample x-y plane travel limit switch design .................................................. 49

4.2.3 Precise sample position registration ............................. 51

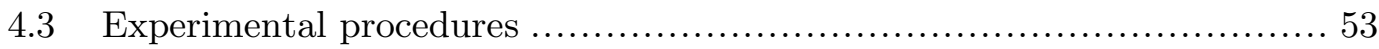

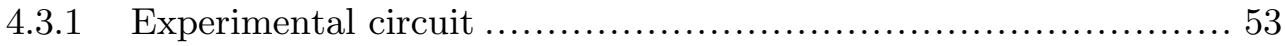

4.3.2 Entire surface $\operatorname{scan}$............................................. 55

4.3.3 Local scan and characterization of emitting sites ................. 58

4.3.4 Computerized operation and graphical user interface............. 62

4.3.5 In situ examination and material characterization of emitters ..... 63

5 Enhanced field emission from chemically etched niobium .................6 64 
5.1 Chemical etching process and chemistry ............................. 64

5.2 Summary of preliminary test results................................... 65

5.2.1 Categories of observed emitters .............................. 66

5.2.2 Statistics of emitters ............................................ 71

5.3 Results after improvement in machining process....................... 72

5.3.1 Improvement in machining process ............................. 72

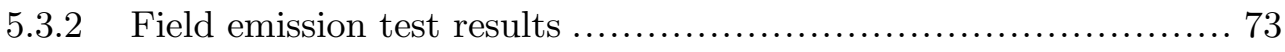

5.3.3 Field emission test results after further improvement in sample preparation ................................................. 75

5.4 Conclusions and discussion ........................................ 79

6 Enhanced field emission from electropolished niobium.................... 82

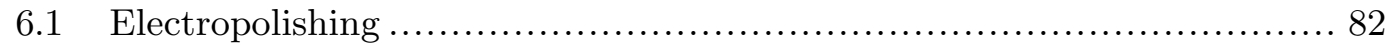

6.1.1 Motivation: Nb cavity performance improvement by electropolishing over chemical polishing ........................ 82

6.1.2 Electropolishing mechanism ................................ 84

6.1.3 Electropolishing setup and chemistry for $\mathrm{Nb} \ldots \ldots \ldots \ldots \ldots \ldots \ldots . \ldots . \ldots 5$

6.2 Field emission results from electropolished niobium ..................... 86

6.3 Comparison between chemically etched and electropolished Nb samples .. 89

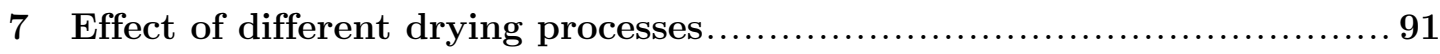

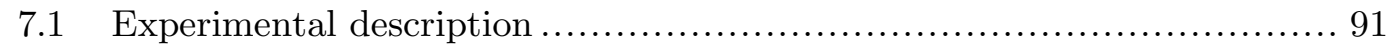

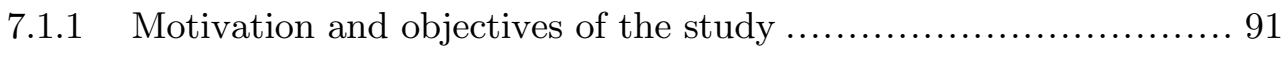

7.1.2 High pressure ultra pure water rinse........................... 92

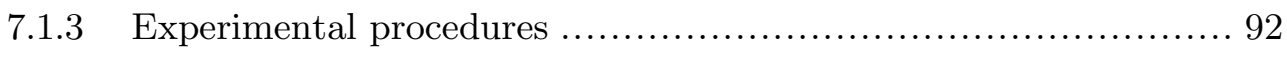

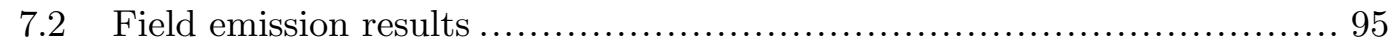

7.3 Discussion........................................................ 95

8 Enhanced field emission from energetically deposited niobium thin film . 99

8.1 Energetically deposited niobium film................................. 99 
8.2 Enhanced field emission studies on energetically deposited Nb thin film..101

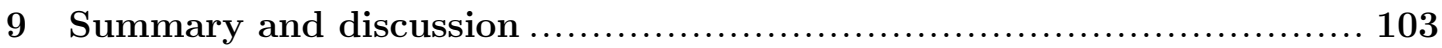

9.1 Discussion on field emission physical mechanism ....................... 103

9.1.1 Various models-supporting and refuting evidence................. 103

9.1.2 The role of surface adsorbate in field emission .................... 105

9.2 Possible experiments and potential application for the apparatus ......... 107

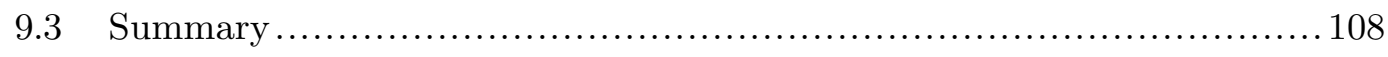

A Illustration of LabView control panel and block diagram for field

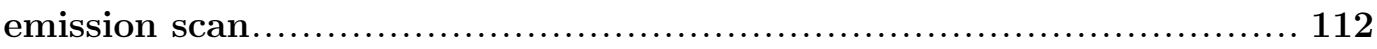

B Illustration of GaAs photocathode field emission scan and identified

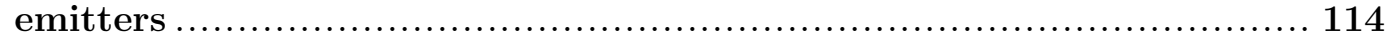

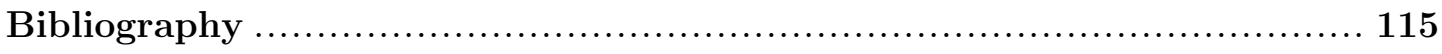




\section{List of Tables}

5.1: A small selection of analyzed emitters from the initial 20 samples after $150 \mu \mathrm{m}$ - $450 \mu \mathrm{m}$ removal by BCP unless otherwise noted. \#9-1(3), e.g., is from sample \#9, after the 1 st surface removal $\left(2^{\text {nd }}, 3^{\text {rd }}, \ldots .\right.$. is after additional removal $)$, on

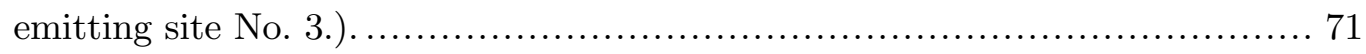

5.2: Field emitters from BCP samples after improvement in machining (Field emission scanning field $140 \mathrm{MV} / \mathrm{m}$, indicated fields are the emitting fields that yield $2 \mathrm{nA} \mathrm{FE}$ current, the scan area for each sample is $25 \mathrm{~mm}$ dia.) ............ 73

5.3: Field emission results at $\sim 140 \mathrm{MV} / \mathrm{m}$ from BCP prepared samples after further improvement in machining (the scan area for each sample is $25 \mathrm{~mm}$ dia.) ...... 76

6.1: Field emission results from electropolished Nb samples (scanning field 140 $\mathrm{MV} / \mathrm{m}$, scan area per sample is $25 \mathrm{~mm}$ dia.) 88

9.1: Relevant findings from various institutions. (Listed under University of Aston is Latham and co-workers' findings). $\sqrt{ }$ : yes, $\mathrm{x}$ : no. ............................ 104 


\section{List of Figures}

1.1: A pair of $1500-\mathrm{MHz}, 5$-cell niobium SRF cavities for CEBAF $\ldots \ldots \ldots \ldots \ldots \ldots \ldots \ldots \ldots \ldots \ldots \ldots$

1.2: A schematic illustration of electric and magnetic field distributions in a typical

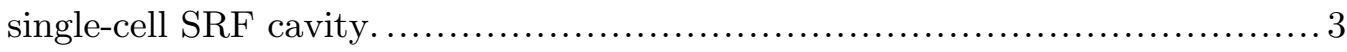

1.3: Surface electric field and magnetic field as a function of distance from equator

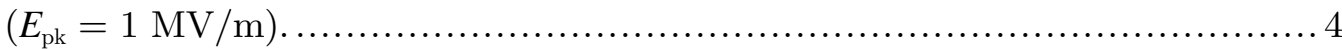

1.4: A schematic illustration of $Q_{0}$ vs. $E_{\text {acc }}$ for a cavity limited by field emission load at high field. ............................................................... 6

2.1: Electrostatic potential and electron wave function at a metal surface with an applied field................................................................... 10

2.2: A schematic illustration of field emission current instability and the conditioning effect of a high voltage vacuum gap. 12

2.3: A schematic illustration of multistable switching recorded by Ph. Niedermann

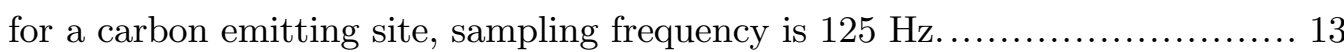

2.4: A schematic illustration of hysteresis in current-voltage characteristics of a high voltage gap. .......................................................... 14

2.5: Light spectra recorded at (a) k-spots and (b) anode spots by Hurley........... 15

2.6: A schematic comparison of electron spectra from emitting sites on broad-area copper, stainless steel and titanium cathodes to that from a micropoint tungsten cathode. .................................................... 16

2.7: A schematic illustration of current-voltage characteristics of prebreakdown and breakdown phenomenon................................................ 17

2.8: Geometrical enhancement factor $\beta$ calculated for various geometries: 
sphere, cylinder and ellipsoid on top of a flat surface.

2.9: The superposed geometrical protrusions to explain the field enhancement

factor.

2.10: Band diagram for MIV model.

2.11: Band diagram for a metal-insulator system involving band bending due to the accumulation of space charges with applied electric field.

2.12: The MIM model (antenna model), with a thin conducting flake on top of an insulating layer. The equipotential lines of the electric field are schematically drawn.

2.13: Filament model proposed by Hurley, involving a certain semiconducting or insulating thin layer on top of metal. ...

2.14: Resonant tunneling model: a local energy level is created due to adsorbate on top of a metal-insulator interface. 26

2.15: Thermionic emission in a metal-insulator-vacuum system.

3.1: Average number of emitting sites per sample through a heating cycle at 90 $\mathrm{MV} / \mathrm{m}$

3.2: Features found at field emission sites in SRF cavities, showing starburst and tracking (left) and craters and ripples (right)....

3.3: Emitting sites density for various purity of niobium, the higher the RRR, the purer the material. All localized emitting sites were particles containing $\mathrm{Nb}$ and Fe. (6 samples, BCP 1:1:1 removal of $\sim 170 \mu \mathrm{m}$, high pressure ( 80 bar) ultra-pure $(17.5 \mathrm{M} \Omega \mathrm{cm})$ water rinsing, electronic grade methanol rinsing). 37

3.4: Statistics of emitter density reported by the University of Wuppertal. 37

4.1: A schematic illustration of known and potential emitters on material surface.

4.2: Grain boundary geometry from chemically etched (by BCP), electropolished and UHV fired (heat treated, $\sim 2100^{\circ} \mathrm{C}$ ) $\mathrm{Nb}$ surfaces. 
4.3: Program to categorically remove field emitters. (BCP: buffered chemical polishing, UWR: ultrasonic water rinse, HPWR: high pressure water rinse, EP: electropolishing, HT: heat treatment at high temperature $\left(\sim 1400^{\circ} \mathrm{C}\right) \ldots . .43$

4.4: Top view of experimental apparatus......................................... 45

4.5: The position of the long distance optical microscope relative to sample and anode tip in SFEM chamber. .................................................. 45

4.6: Three artificial marks on sample surface are used to correlate $x, y$ coordinates in SFEM with $x, y$ coordinates in SEM.................................... 47

4.7: $\quad$ Sample heat treatment by electron bombardment........................... 48

4.8: Picture of the apparatus. ................................................. 49

4.9: The setup of stepper motor travel limit switch. ............................ 50

4.10: The hardware for the stepper motor control................................ 51

4.11: The methodology to distinguish external particles from material impurities. A foreign microparticle is shown at the left $(E=136 \mathrm{MV} / \mathrm{m}$, containing $\mathrm{Nb}, \mathrm{Fe}$, $\mathrm{Cr}, \mathrm{Ni}$ ). After ultrasonic water rinse it disappeared (right). .................. 53

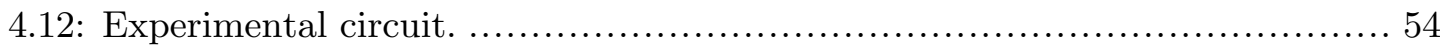

4.13: Illustrative interpolation and extrapolation of a nine-point profile data set to

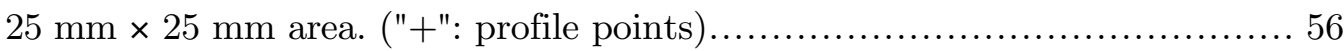

4.14: Correction factor $\kappa(=V /(E \cdot d))$ versus $d / r$ for hyperboloid-plane (a) and

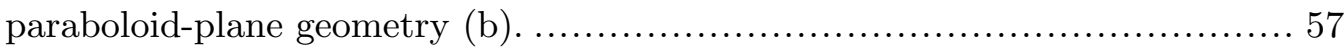

4.15: The lateral distribution of electric field on sample for paraboloid-plane geometry.

4.16: Electric field lateral distribution measured for the $10 \mu \mathrm{m}$ radius anode at 100 $\mu \mathrm{m}$ gap. 59

4.17: Illustration of coarse scan of an entire sample surface $(\phi 25 \mathrm{~mm})$ at $140 \mathrm{MV} / \mathrm{m}$ using a cylindrical anode of $150 \mu \mathrm{m}$ radius (left), along with local scan of 100 $\mu \mathrm{m} \times 100 \mu \mathrm{m}$ area at the center of the outlined emitter using an anode of $1 \mu \mathrm{m}$

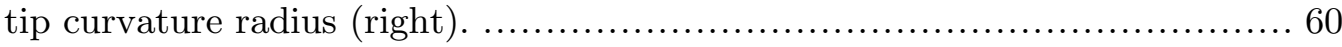


4.18: Calibration of gap and electric field at the emitting site. Data shown is obtained using a paraboloid-shaped anode of $10 \mu \mathrm{m}$ radius.

4.19: The calibration of field correction factor $\kappa$ using the cylindrical anode of 150 $\mu \mathrm{m}$ radius at the same emitting site and adjusting the voltage to obtain the

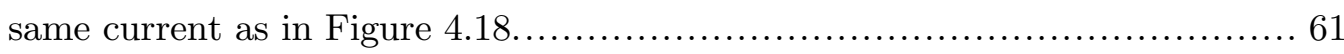

4.20: $\beta, S$ fitting for the characterization of emitter. 62

4.21: Schematic illustration of the typical probing depth of SEM and EDX in a lowdensity low-atomic-number target.

5.1: Geometrical damages as field emitters. The emitting field (for $2 \mathrm{nA}$ detected field emission current), elemental composition detected by EDS, and in some cases the F-N fitting parameters $\beta$ and $S$ of emitters are listed.............. 67

5.2: Some pictures of foreign particles or contaminants as field emitters. ........... 68

5.3: Emitting features with no foreign elements detected by EDS................. 69

5.4: Geometrical irregularities as field emitters, no foreign elements detected...... 70

5.5: An illustrative spectrum of EDS at one emitting site. EP: eletropolishing..... 72

5.6: A selection of SEM pictures of emitters listed in Table 5.2 and 5.3. ........... 77

5.7: A selection of SEM pictures of emitters listed in Table 5.2 and 5.3. ........... 78

6.1: The statistics of achieved acceleration gradient on a number of $\mathrm{Nb}$ cavities by chemical etching and by electropolishing at CERN. .......................... 83

6.2: The comparison of $\mathrm{BCP}$ and $\mathrm{EP}$ on the same cavity ....................... 83

6.3: Mechanism of electropolishing........................................... 85

6.4: The schematic setup for electropolishing of Nb......................... 87

6.5: Comparison of BCP etched and electropolished Nb sample surface............ 87

6.6: A foreign particle emitter from sample \#65-1 (left, the craters are caused by vacuum arc, the particle contains $\mathrm{Nb}, \mathrm{Fe}, \mathrm{Cr}, \mathrm{E}=105 \mathrm{MV} / \mathrm{m}$ ) and an arc site from sample \#63-1 (right, the small particles are generated by vacuum arc)

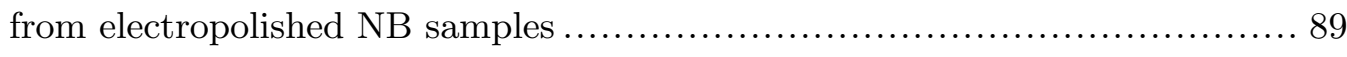

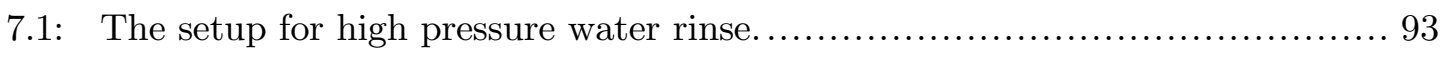


7.2: The experimental procedures for the comparison of drying processes.

7.3: Field emission results for the comparison of drying processes. Sample \#71, \#72 and \#84 is in row 1, 2 and 3 respectively. The first column is for "ultrasonic rinse", the second is for "quick drying", and the third is for "slow

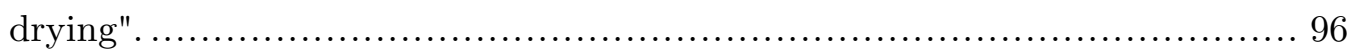

7.4: SEM pictures of emitters from sample \#84, left is after "quick drying", right is after "slow drying". EDS didn't detect any foreign elements. ................. 97

8.1: Illustration of surface atom nucleation: column forming in relation to atom mobility ................................................................... 100

8.2: Illustration of the energetic deposition system by ECR in vacuum. ............ 100

8.3: One emitter located on energetically deposited $\mathrm{Nb}$ thin film. The pictures are from the same site, but at different magnification. The crater was generated by vacuum arc. It contains $\mathrm{Cu}, \mathrm{Fe}, \mathrm{Ni}$. Cr. Note the starburst and tracking

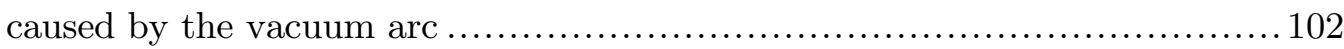

9.1: Density of field emission sites identified in DC (Geneva and Wuppertal) and RF studies (CERN and Cornell) (i.e., density of emitters with $E_{\mathrm{onset}} \leq E_{\mathrm{pk}}$ identified in a DC scan or an RF operation at $E_{\mathrm{pk}}$ surface field versus $\left.E_{\mathrm{pk}}\right)$, with the addition of this work (TJNAF) 


\section{Chapter 1}

\section{Introduction}

\subsection{Motivation for the studies}

\subsubsection{Superconducting RF cavities}

Particle accelerators are the essential tools used to answer questions in nuclear and high energy physics, such as on the fundamental constituents of matter, quarks and leptons. As accelerator technology developed over the years, the frontiers in beam energy and beam luminosity have been continuously pushed ahead. For $\mathrm{e}^{+} \mathrm{e}^{-}$ accelerators, the record for beam energy was set by the LEP-II $\mathrm{e}^{+} \mathrm{e}^{-}$accelerator at CERN ( 100 GeV per beam); however, the TESLA (TeV Superconducting Linear Collider) project has been proposed, and it will push the center-of-mass energy to 1 $\mathrm{TeV}$. The TESLA project relies on the technology of superconducting radiofrequency (SRF) to meet its design goals in beam energy and beam current; as do some other future projects, for example, the LHC (Large Hadron Collider) at CERN and the muon collider. The technology of SRF has matured over the past few decades, and is currently employed in CEBAF (Continuous Electron Beam Acceleration Facility) at TJNAF (Thomas Jefferson National Accelerator Facility), HERA at DESY, KEK-B factory at KEK, CESR (Cornell Electron Storage Ring) at Cornell, SNS (Spallation Neutron Source) at Oak Ridge National Lab, etc. An example of typical SRF cavities - the acceleration unit in SRF accelerator is illustrated in Figure 1.1. 


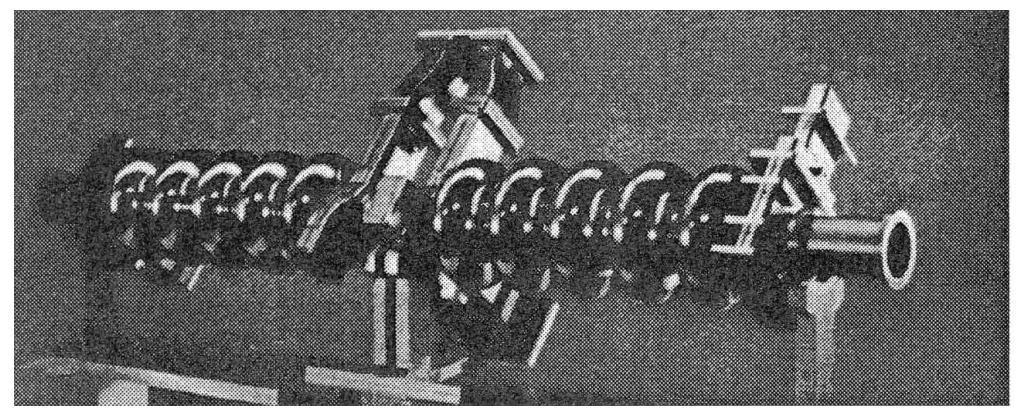

Figure 1.1: A pair of 1500-MHz, 5-cell niobium SRF cavities for CEBAF.

Compared to normal conducting copper cavities, superconducting cavities (made of niobium or lead) consume orders of magnitude less rf power, and at least two orders of magnitude less total power in continuous wave (cw) applications even after taking into account the power for refrigeration. This reduced power demand also enables the SRF cavity to operate at a higher acceleration gradient, for example, 10 $\mathrm{MV} / \mathrm{m} \mathrm{cw}$ has been routinely achieved, surpassing that of copper cavities. Furthermore, the large apertures afforded by SRF cavities reduce the disruptive interactions between beam and cavities, making them more suitable for high current operation. As a result, SRF technology is foreseen to play a key role in future generations of particle accelerators. ${ }^{1}$

Refer to Figure 1.2 for a schematic illustration of electric and magnetic field distributions in a typical SRF cavity in the $\mathrm{TM}_{010}$ mode (the mode for acceleration in most SRF cavities). The electric field is strongest along the cavity axis (hence chosen as the beam line) and at the iris region, and is weakest at the equator, while the magnetic field is strongest at the equator and becomes weaker near the irises. The surface electric field and magnetic field inside the SRF cavity is shown in Figure 1.3 as a function of distance from the equator. Peak electric surface field $\left(E_{\mathrm{pk}}\right)$ occurs at the iris, and peak magnetic surface field $\left(H_{\mathrm{pk}}\right)$ occurs near the equator. 

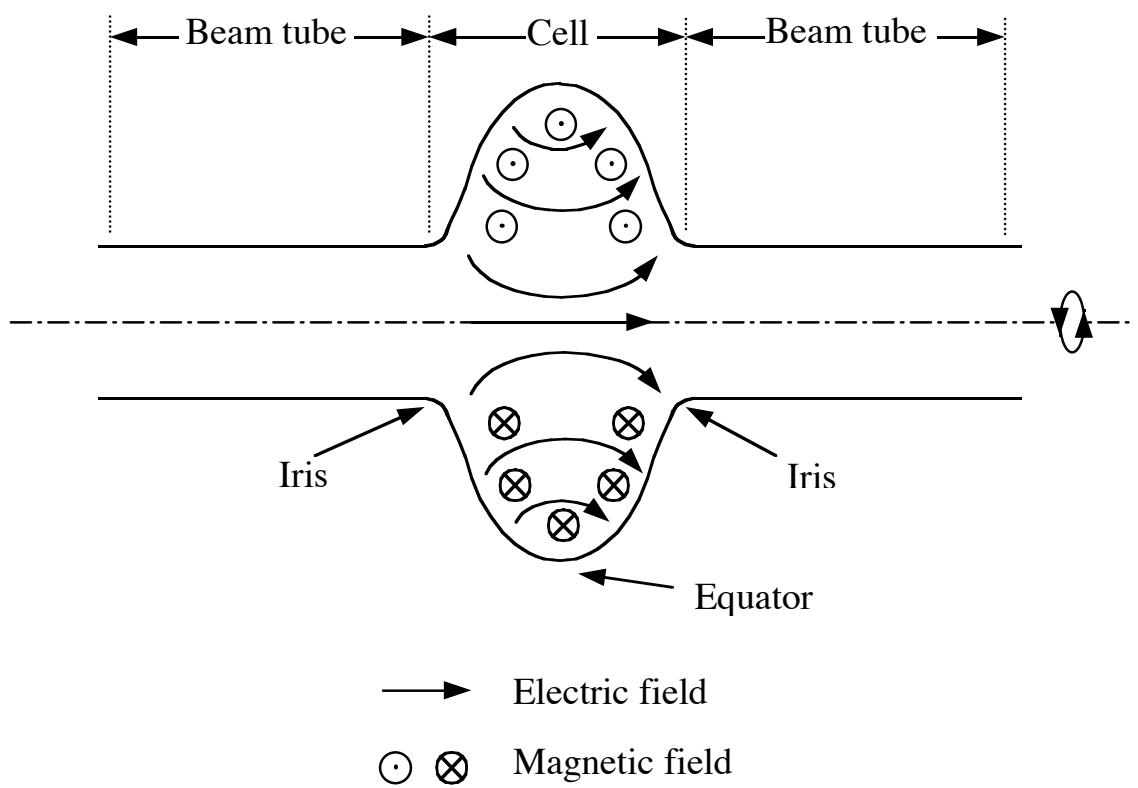

Figure 1.2: A schematic illustration of electric and magnetic field distributions in a typical single-cell SRF cavity.

The electron is accelerated by the electric field when entering the SRF cavity along its axis. Energy gained by electron with speed near that of light in each cavity is $e \cdot V$, where

$$
V=\int_{0}^{l} E(z) e^{i \omega_{0} z / c} d z
$$

where $E(z)$ is the electric field along cavity axis, $\omega_{0}$ is the operation frequency of the cavity, $l$ is the length of the acceleration region and $c$ is the speed of light. Therefore, the acceleration gradient $\left(E_{\text {acc }}\right)$ in each acceleration unit is given by

$$
E_{a c c}=\frac{V}{l}=\frac{1}{l} \cdot \int_{0}^{l} E(z) e^{i \omega_{0} z / c} d z
$$

To achieve maximum performance in cavities, it is often necessary to minimize the ratios of $E_{\mathrm{pk}} / E_{\mathrm{acc}}$, and $H_{\mathrm{pk}} / E_{\mathrm{acc}}$ by cavity shape optimization, as the two peak fields can determine the operating limit of cavities. Theoretically, the maximum accelerat- 

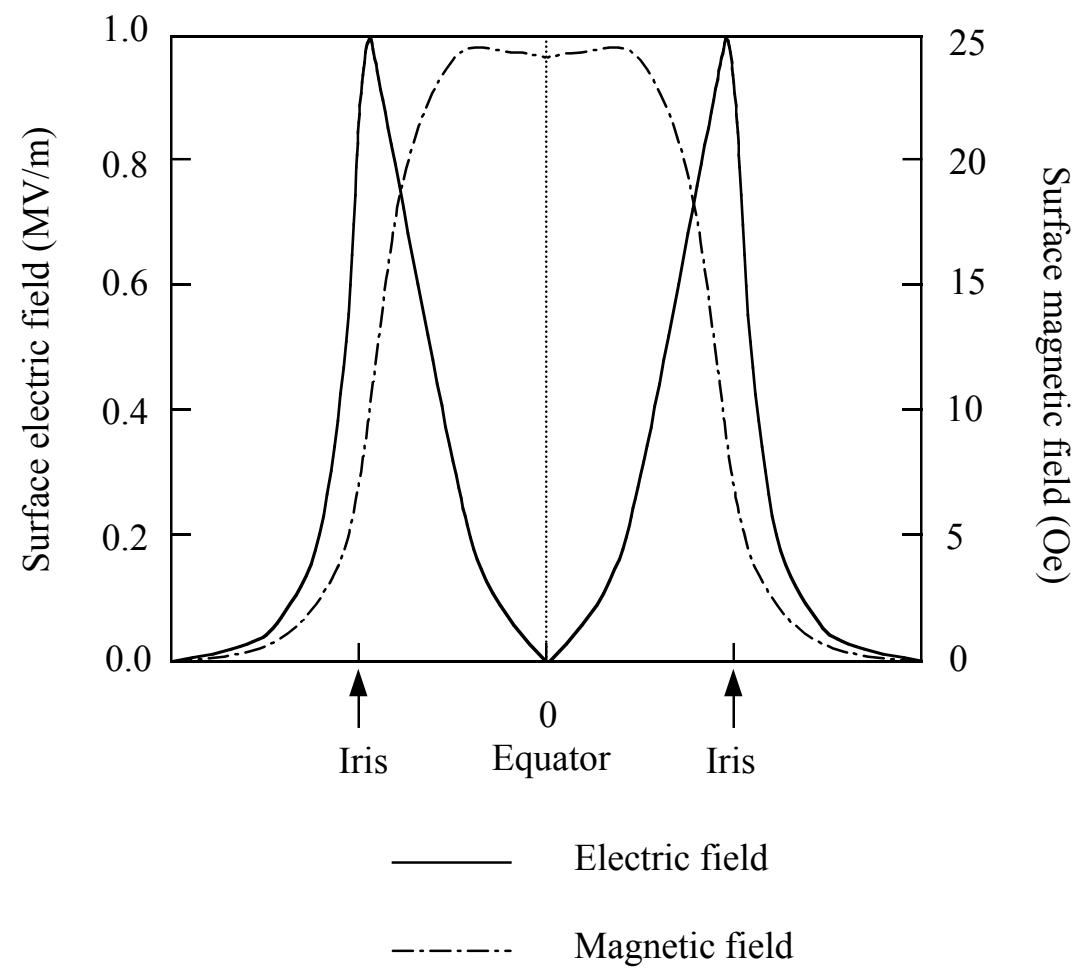

Figure 1.3: Surface electric field and magnetic field as a function of distance from equator $\left(E_{\mathrm{pk}}=1 \mathrm{MV} / \mathrm{m}\right)$.

-ion gradient in SRF cavities should be limited only by the superheating critical magnetic field $\left(H_{\mathrm{sh}}\right)$, where

$H_{\mathrm{sh}}>H_{\mathrm{c}},\left(H_{\mathrm{c}}\right.$ : critical magnetic field for type I superconductor)

$H_{\mathrm{sh}}>H_{\mathrm{c} 1},\left(H_{\mathrm{c} 1}\right.$ : lower critical magnetic field for type II superconductor, e.g., Nb, $\left.\mathrm{Nb}_{3} \mathrm{Sn}\right) .^{2}$

If $H_{\mathrm{sh}}$ is exceeded at the cavity surface, the superconductor will become normal conducting (quench). For niobium, the superheating critical field expected from theory is approximately 2200-2400 Oe. ${ }^{3}$ For the usual cavity shape, the ratio of $H_{\mathrm{pk}} / E_{\text {acc }}$ is typically $\approx 50 \mathrm{Oe} / \mathrm{MV} / \mathrm{m}$, thus the corresponding theoretical limit on acceleration gradient $\left(E_{\text {acc }}\right)$ would be approximately $50 \mathrm{MV} / \mathrm{m} .{ }^{1}$ At this gradient, the 
peak surface electric field is approximately $100 \mathrm{MV} / \mathrm{m}$, as $E_{\mathrm{pk}} / E_{\mathrm{acc}}$ is typically between $1.8-2{ }^{1}$

However, this gradient has never been achieved in practice due to thermal breakdown (quenching) or enhanced field emission (EFE) or multipacting. Thermal breakdown is usually caused by local surface defects or foreign particles which exhibit a high surface resistance. Field emission is the tunneling of electron under high surface electric field from a localized area on a cavity wall into the cavity and then being accelerated by the electromagnetic field. RF power is lost to the emitted electron, and when electrons hit the cavity wall, heat is generated locally, which further increases the losses and may induce quenching, and x-rays are produced. Field emission current is observed to increase exponentially with electric field. Refer to Figure 1.4 for an illustration of $Q_{0}$ vs. $E_{\text {acc }}$ for a cavity that is limited by field emission at high fields, where $Q_{0}$ is the cavity quality factor. $Q_{0}$ is defined as

$$
Q_{0}=\frac{\omega_{0} U}{P_{d}}
$$

where $U$ is the total energy stored in the cavity and $P_{\mathrm{d}}$ is the power dissipated on the entire cavity interior surface. i.e., $Q_{0}$ is $2 \pi$ times the extrapolated number of rf cycles needed to dissipate all the energy stored in cavity.

Multipacting is the resonant impact of electrons in an RF cavity. It is generally agreed that the process originates with ejected electrons from the cavity wall, which are then accelerated by resonant rf fields and hit another location on the cavity wall, producing secondary electrons. Subsequently, the secondary electrons are accelerated and hit the cavity wall again producing another generation of secondary electrons. As the cycle repeats, an avalanche of electrons is generated and absorbs a significant amount of $\mathrm{rf}$ power. As a result, the gradient fails to increase with $\mathrm{rf}$ power, and the heat generated by electron impact could initiate thermal breakdown. 


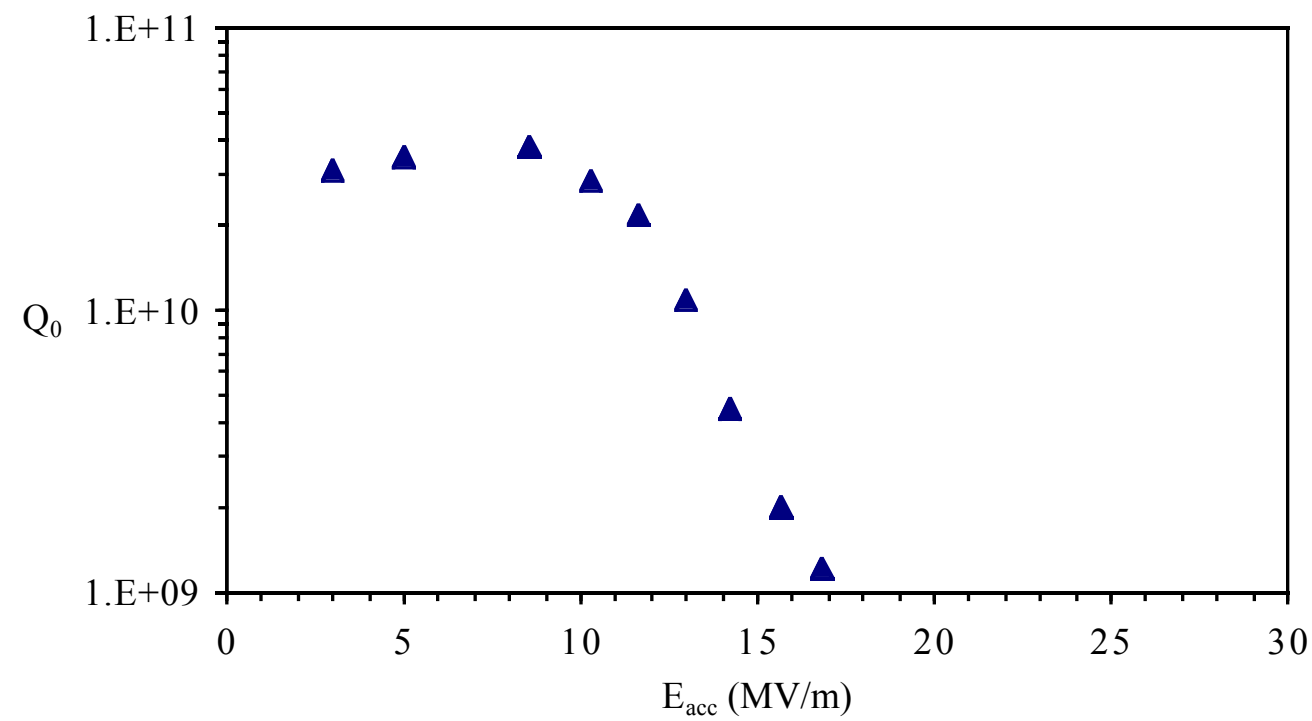

Figure 1.4: A schematic illustration of $Q_{0}$ vs. $E_{\text {acc }}$ for a cavity limited by field emission load at high field.

During the past years, the improvement of superconducting cavity preparation, dust free assembly and processing techniques have increased the acceleration gradient of superconducting $\mathrm{RF}$ cavities to $E_{\text {acc }} \geq 20 \mathrm{MV} / \mathrm{m}$, but the reproducibility and further increase of gradient still suffer from the breakdown of superconductivity induced by EFE and quenching. ${ }^{1,4,5}$ Field emission remains the primary limitation after the effective suppression of quenching by using $\mathrm{Nb}$ of high thermal conductivity.

EFE also limits the performance of klystrons in the RF regime. Klystrons are usually the rf power sources used to drive rf cavities.

To study the causes of field emission on $\mathrm{Nb}$ and to search for ways to reduce or eliminate it, are the primary motivations for this thesis project. 


\subsubsection{High voltage vacuum devices and other applications}

Enhanced field emission is also a fundamental problem in a wide range of high voltage vacuum devices working in the DC regime. For instance, EFE causes sparking problem in vacuum switches. Field emission, when utilized as a source of electrons in cold cathode devices, has applications in flat panel display and other microelectronic devices. Since the fundamental mechanism of EFE is not expected to differ significantly from one solid metal to another, the conclusion obtained from $\mathrm{Nb}$ should be applicable to the above devices as well.

\subsection{Organization of the dissertation}

In chapter 2, the fundamental FE theory and modified theory for EFE are introduced. Some general characteristics established and associated with EFE through previous experiments by others are then discussed. Finally, some models proposed for EFE from DC or RF research will be described.

Field emission research relevant to $\mathrm{Nb}$ has been conducted in a number of institutions around the world. Ph. Niedermann from University of Geneva was the first one to set up a DC field emission scanning apparatus to combine FE scanning with in situ microscopic observation and material characterization of emitters; and he carried out an extensive study on Nb. Researchers at Centre d'Etudes de Saclay (Saclay) and Institut de Physique Nucleaire (IPN) Orsay France modified a commercial Scanning Electron Microscope (SEM) as a FE scanning apparatus at the $\mathrm{nA}$ level and also built a sensitive device to detect $\mathrm{FE}$ current well below $1 \mathrm{nA}$. Cornell University examined the inner surface of an SRF cavity after the occurrence of field emission. They also built a special "mushroom" shaped cavity to test FE on samples mounted inside of the cavity; and the sample could then be detached for SEM observation and material characterization. University of Wuppertal built a similar apparatus to that of the University of Geneva, and conducted research on $\mathrm{Nb}, \mathrm{Cu}$, etc. Findings and $\mathrm{FE}$ performance achieved by the above institutions are 
summarized in chapter 3. Most of the previous work on this subject was done before the availability of high pressure water rinse, and most of the previous studies have been discontinued.

Chapter 4 starts with the experimental objective of this project and is devoted to a description of the apparatus the author built at Jefferson Lab. The design, construction of the apparatus and operating procedures are described in detail.

Chapters 5, 6, 7, 8, 9 are devoted entirely to the experimental results and observations by the author in this project. From the most commonly used $\mathrm{Nb}$ surface preparation-chemical etching by BCP (Buffered Chemical Polishing) to the most recently re-discovered electropolishing, the results are presented and compared in chapter 5 and 6 . After emission-free or near-emission-free surfaces have been consistently achieved on chemically etched or electropolished samples at 140 $\mathrm{MV} / \mathrm{m}$, a comparative study is done on the drying processes due to concerns and speculations that the natural drying process may produce or introduce new and unique emitters. The results are covered in Chapter 7. Nb thin film, for example, energetically deposited on $\mathrm{Cu}$ substrate developed by Genfa Wu in a parallel thesis project at TJNAF, presents a possible alternative to the customary bulk Nb cavity. The EFE performance of a first such sample is tested and described in chapter 8 .

Chapter 9 summarizes the experimental results, provides an outlook for future applications for the apparatus and discusses the physical mechanisms of field emission. 


\section{Chapter 2}

\section{Field Emission Fundamentals}

In this chapter, we will focus on the theoretical aspects of field emission, beginning with Fowler-Nordheim theory and modified Fowler-Nordheim theory, followed by general characteristics associated with enhanced field emission and ending with several models proposed from previous work by others. The experimental aspects of prior work which focused on $\mathrm{Nb}$ will be introduced in chapter 3 .

\subsection{Field emission theory and enhanced field emission}

\subsubsection{Fowler-Nordheim theory}

Field emission of electrons occurs when electrons tunnel through the surface barrier of a metal into vacuum under high electric field. The emission from a clean metal surface was explained by Fowler and Nordheim in terms of quantum mechanical tunneling effect in 1928. ${ }^{6,7}$ Fowler-Nordheim (F-N) law has been subjected to fairly extensive verification and is considered to be well established on experimental and theoretical grounds. As shown is Figure 2.1, the surface potential barrier has an approximately triangular shape determined by the work function $\phi$ of the solid and the electric field $E$.

By solving the Schrödinger equation for the triangular potential barrier, they obtained the electron tunneling probability with an applied electric field. The emission current density is also obtained by integrating the transmission probability 


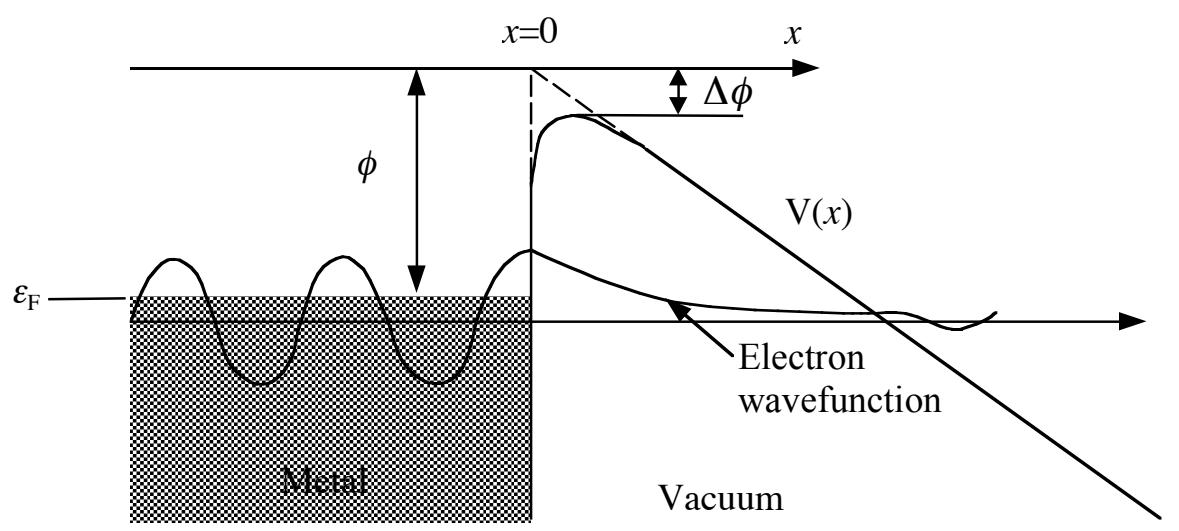

Figure 2.1: Electrostatic potential and electron wave function at a metal surface with an applied field.

and the incident electron flux at each energy over the entire energy range. The result is the so-called Fowler-Nordheim law:

$$
j=\frac{A \cdot E^{2}}{\phi} \cdot \exp \left(-\frac{B \cdot \phi^{\frac{3}{2}}}{E}\right) .
$$

With current density $j$ in Amp $/ \mathrm{cm}^{2}$, electric field $E$ in $\mathrm{V} / \mathrm{cm}$ and work function $\phi$ in $\mathrm{eV}(\phi=4 \mathrm{eV}$ for $\mathrm{Nb})$, we have $A=1.54 \times 10^{-6} \mathrm{eV} \cdot \mathrm{Amp} / \mathrm{V}^{2}, B=6.83 \times 10^{7}(\mathrm{eV})^{-1.5}$. $\mathrm{V} / \mathrm{cm}$.

If the image force, i.e., the attractive force between an electron and a conducting surface, which can be represented by a positive charge opposite to the electron, is considered, the electrostatic potential becomes ${ }^{6,7,8}$

$$
V(x)=-\frac{e^{2}}{4 \cdot 4 \pi \varepsilon_{0} \cdot x}-e \cdot E \cdot x .
$$

The maximum of this potential occurs when the image force portion equals the electric field portion, i.e.,

$$
-\frac{e^{2}}{4 \cdot 4 \pi \varepsilon_{0} \cdot x_{0}}=-e \cdot E \cdot x_{0} \Rightarrow x_{0}=\sqrt{\frac{e}{16 \pi \varepsilon_{0} E}} .
$$

By substituting $x_{0}$ into Eq. 2.2, one can obtain the potential barrier lowering $\Delta \phi$ : 


$$
\Delta \phi=e \cdot \sqrt{\frac{e \cdot E}{4 \pi \varepsilon_{0}}} .
$$

Refer to Figure 2.1 for the barrier shape and wave function. The Schrödinger equation may be solved via the WKB approximation. ${ }^{9}$ The result is that the current density can be expressed by

$$
j(E)=\frac{A \cdot E^{2}}{\phi \cdot t^{2}(y)} \cdot \exp \left\{-B \cdot \frac{\phi^{\frac{3}{2}}}{E} \cdot v(y)\right\},
$$

where $t(y)$ and $v(y)$ are two slowly varying functions of $y(=\Delta \phi / \phi) .{ }^{10}$ To a good approximation, ${ }^{11} t^{2}(y)=1.1$, and $v(y)=0.95-y^{2}$. The functional form of the field emission current then simplifies to

$$
I=a \cdot E^{2} \cdot \exp \left\{-\frac{b}{E}\right\}
$$

with $\mathrm{a}$ and $\mathrm{b}$ given by

$$
\begin{gathered}
a=\frac{A \cdot S}{1.1 \cdot \phi} \cdot \exp \left\{\frac{B \cdot 1.44 \cdot 10^{-7}}{\phi^{\frac{1}{2}}}\right\} \\
b=0.95 \cdot B \cdot \phi^{\frac{3}{2}}
\end{gathered}
$$

and $S$ as the total emitting area in $\mathrm{cm}^{2}$. According to the Fowler-Nordheim equation, the FE current should only approach $1 \mathrm{~A} / \mathrm{cm}^{2}$, at surface fields of $\approx 1900$ $\mathrm{MV} / \mathrm{m}$.

\subsubsection{Enhanced field emission}

In practice, FE current is measured at fields much lower than that described by the F-N law on a clean metallic surface, and this phenomenon is called enhanced field emission (EFE). In order to interpret EFE by the F-N law, a field enhancement factor $\beta(>1)$ is introduced and usually leads to a good approximation. Since it is unknown if an image force affects EFE, the modified F-N law is simply based on the triangular potential barrier (without image force correction), i.e., 


$$
I=\frac{A \cdot S \cdot \beta^{2} \cdot E^{2}}{\phi} \cdot \exp \left(-\frac{B \cdot \phi^{\frac{3}{2}}}{\beta \cdot E}\right) .
$$

$\beta$ (generally ranges from 50 to 1000) and $S$ (the emitting surface area, generally ranging between $10^{-14}-10^{-5} \mathrm{~cm}^{2}$ but sometimes exhibiting an unreasonable value, from $10^{-18}$ to $\left.1 \mathrm{~cm}^{2}\right)^{12,13}$ can be obtained from the slope and the intercept by fitting the $\ln \left(I / E^{2}\right)$ versus $1 / E$ distribution to a straight line. Reviews exist on this subject. ${ }^{14,15}$

\subsection{General characteristics of enhanced field emission}

\subsubsection{Current instability and conditioning effect}

Field emission current generally displays an instability, which can be improved by conditioning. As described by R. V. Latham, ${ }^{15}$ when the applied voltage across a vacuum gap is slowly increased in small steps, the FE current will stabilize with time (Refer to Figure 2.2), showing reductions both in current noise level and in the frequency of the occurrence of current spikes (microdischarge). The time constant of

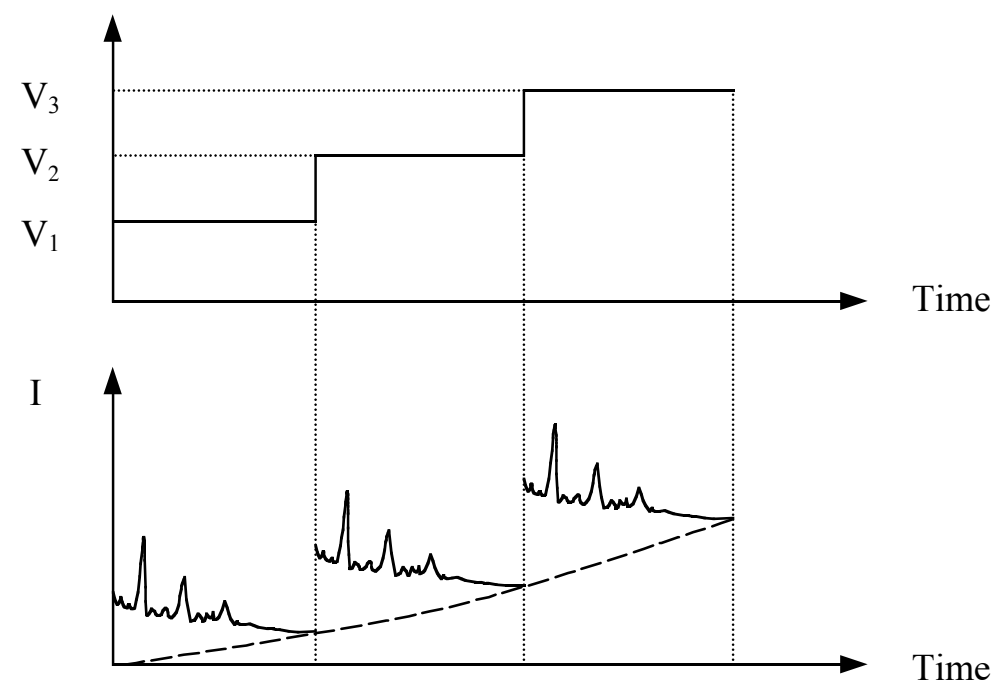

Figure 2.2: A schematic illustration of field emission current instability and the conditioning effect of a high voltage vacuum gap. 
this stabilization process is typically $\approx 30$ minutes. The conditioning effect can be explained by a thermal blunting of sharp protrusions, which will be described in the geometrical enhancement model in next section, or desorption of residual gas induced by high emission current. A third possibility is that the emitter is very hot because the emitting object has very weak thermal conduction to the bulk. This would lead to a combination of field and thermionic emission. Thermionic emitters on superconducting surfaces have been observed, and they have been observed to cool over periods of about 30 minutes, presumably due to sintering of the object to the surface with attendant improvement in thermal conductivity.

\subsubsection{Switching behavior}

Switching behavior in some field emitting sites has been recorded by $\mathrm{Ph}$. Niedermann ${ }^{12}$ and others. The phenomenon is the FE current jumping between two or more constant levels. Refer to Figure 2.3 for the current versus time plot for a carbon emitter.

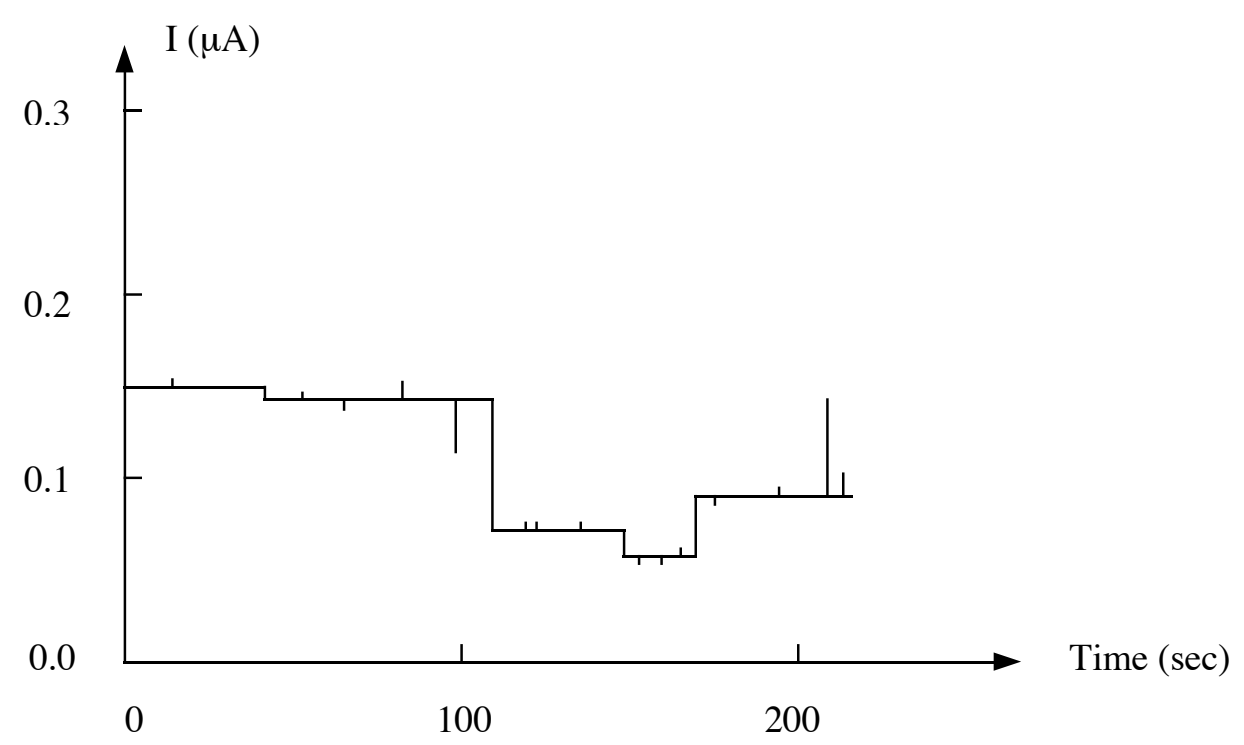

Figure 2.3: A schematic illustration of multistable switching recorded by $\mathrm{Ph}$. Niedermann for a carbon emitting site, sampling frequency is $125 \mathrm{~Hz} .^{12}$ 


\subsubsection{Hysteresis in I-V characteristics}

"Hysteresis" in current-voltage characteristics has been reported by many and is described by R. V. Latham. ${ }^{15}$ At an arbitrary voltage, field emission at some emitting sites can get into an "ignited" stage, in which the current rises by several orders of magnitude and is basically independent of applied voltage. Only when the voltage is lowered even further can the current drop by several orders of magnitude to a previous level. If the voltage is cycled, this hysteresis in I-V characteristics repeats. This behavior is usually associated with the pressure range of $10^{-5}-10^{-4}$ mbar, ${ }^{16,17,18}$ but is observed in UHV environment, too. Refer to Figure 2.4 for an illustration of "hysteresis". The physical explanation of this phenomenon can be made by enhanced field emission from resonance tunneling, which will be described in a later section.

\subsubsection{Light emission}

Light emission, which is sometimes observed from the cathode or anode in a high voltage gap, is called k-spots (cathode spots) or anode spots, respectively. The light

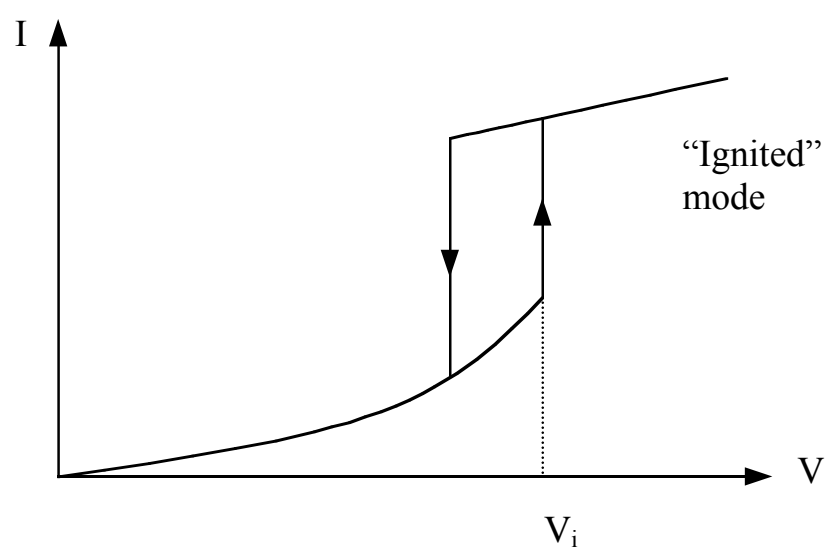

Figure 2.4: A schematic illustration of hysteresis in current-voltage characteristics of a high voltage gap. 
spectra was measured by Hurley ${ }^{19}$ (illustrated in Figure 2.5) and others. K-spots were associated with enhanced field emission sites by Hurley as well. The physical explanation suggests that the k-spots are made by thermal radiation from Joule heated metallic protrusion (refer to following section for the geometrical enhancement model); however, this explanation is not applicable to anode spots. An alternative explanation was proposed by Hurley and Dooley, ${ }^{20}$ invoking a electroluminescent effect from non-metallic emitters, evidenced by the dependence of light emission brightness on voltage.
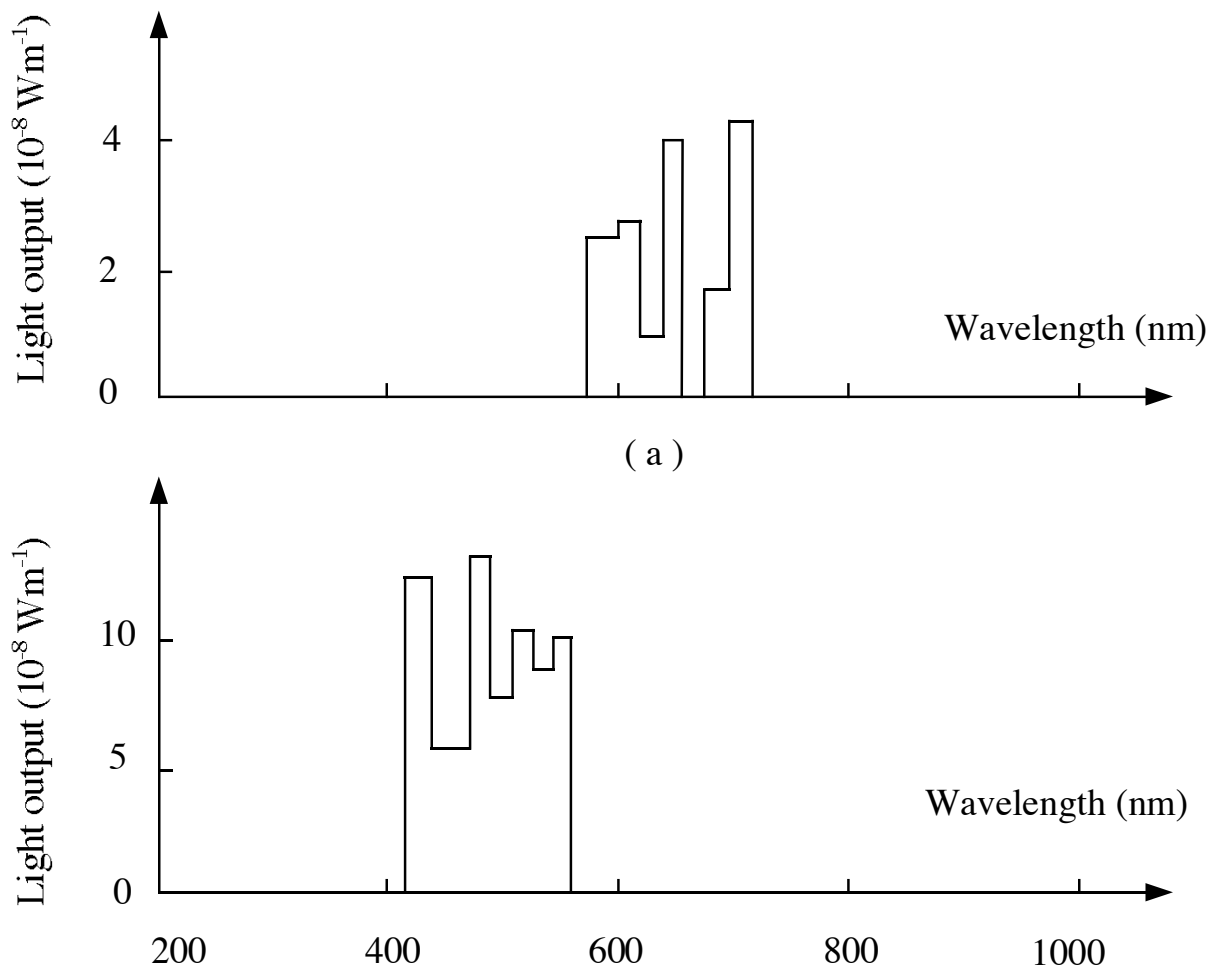

( b )

Figure 2.5: Light spectra recorded at (a) k-spots and (b) anode spots by Hurley. ${ }^{19}$ 


\subsubsection{Electron energy spectra}

Energy spectra of field emitting electrons were measured by Allen and Latham. ${ }^{21}$ Measurement from sites on broad-area copper, stainless steel, and titanium cathodes was made and compared to measurements from a micropoint tungsten emitter. The energy spectra relative to the Fermi level is illustrated in Figure 2.6. It is clearly shown that broad-area emission differs from pure metallic protrusion emission, and models proposed to explain the energy shift and double peaks will be discussed in the following section.

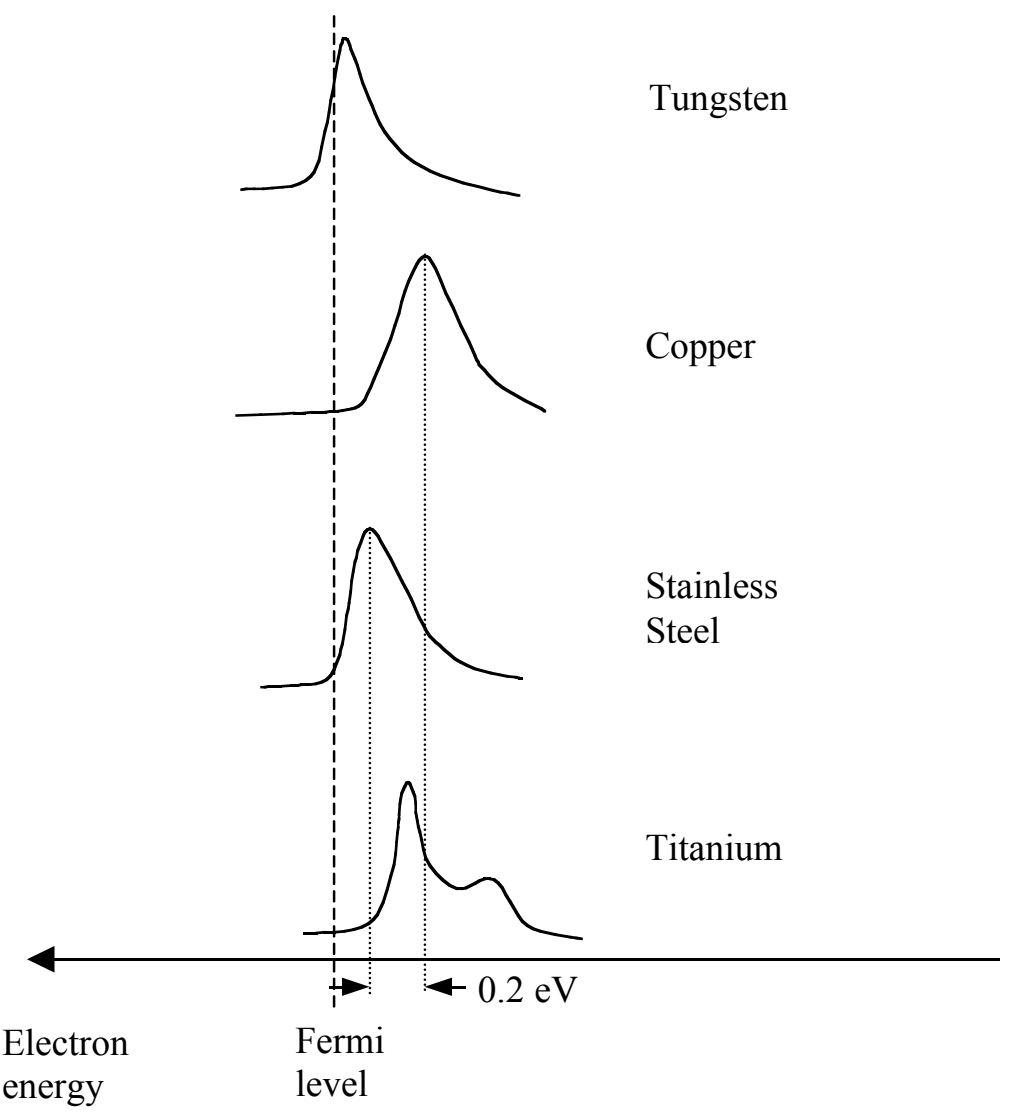

Figure 2.6: A schematic comparison of electron spectra from emitting sites on broad-area copper, stainless steel and titanium cathodes to that from a micropoint tungsten cathode. ${ }^{21}$ 


\subsubsection{Pre-breakdown and breakdown}

On certain emitters, when voltage is increased slowly until a certain current (usually $\geq 0.1 \mathrm{nA}$ ) is detected, the current can rise rapidly with voltage and becomes increasingly unstable, which is called the "prebreakdown" stage; upon further increase of the voltage, an arc can instantly strike between the electrodes and the gap resistance drops to near 0 , which is called vacuum breakdown, vacuum arc or spark. Refer to Figure 2.7 for a schematic illustration of voltage-current characteristics of prebreakdown and breakdown events. Other mechanisms, for instance, involving loosely attached microparticles detached by the electric field and traveling from cathode to anode or vice versa, can cause breakdown as well. This process is discussed by Latham. ${ }^{15}$ Prebreakdown and breakdown are broadly common to all high voltage vacuum gaps.

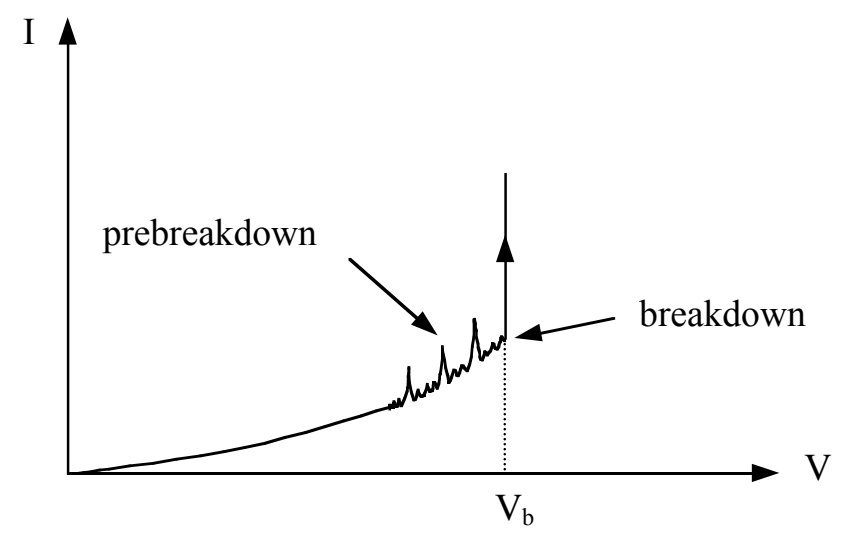

Figure 2.7: A schematic illustration of current-voltage characteristics of prebreakdown and breakdown phenomenon. ${ }^{15}$ 


\subsection{Some models proposed for enhanced field emission}

There are many models proposed to explain EFE. Here we will only discuss some of the most-discussed models.

\subsubsection{Geometrical enhancement model}

Sharp metallic protrusions are believed to cause emission-called geometrical enhancement (protrusion) model-due to the following reasons and experimental findings:

I. The F-N behavior of emissions with the introduction of $\beta$ factor and its independence of temperature change $\left(\mathrm{T} \leq 300^{\circ} \mathrm{K}\right)$. The $\beta$ factor has real meaning in this model. The value of $\beta$ for a semi-elliptical protrusion is calculated by Rohrbach as

$$
\beta=\frac{\left(\lambda^{2}-1\right)^{\frac{3}{2}}}{\lambda \ln \left[\lambda+\left(\lambda^{2}-1\right)^{\frac{1}{2}}\right]-\left(\lambda^{2}-1\right)^{\frac{1}{2}}}
$$

where $\lambda$ is the ratio of semi-major to semi-minor axes of the ellipse, i.e., $\lambda=h / b{ }^{22}$ For very sharp protrusions where $\lambda>>10, \beta$ can be approximated by

$$
\beta \approx \frac{\lambda^{2}}{\ln (\lambda)-0.3} .
$$

$\beta$ values calculated by Rohrbach for various geometries are shown in Figure 2.8. Generally, $\beta$ as a function of $h / r$, with $h$ as the height of the geometry and $r$ as the tip curvature radius, can be expressed to a good approximation, in a unifying equation: ${ }^{23}$

$$
\beta \approx 2+\frac{h}{r}, \text { provided that } \frac{h}{r} \geq 5 .
$$

II. Whisker-like projections were observed on cathodes after a high electric field was applied. Those projections have sharp enough geometry to produce fields of $10^{5}$ $\mathrm{V} / \mathrm{cm} \cdot{ }^{24}$ However, the photograph of the cathode surface edge was taken by an elect- 


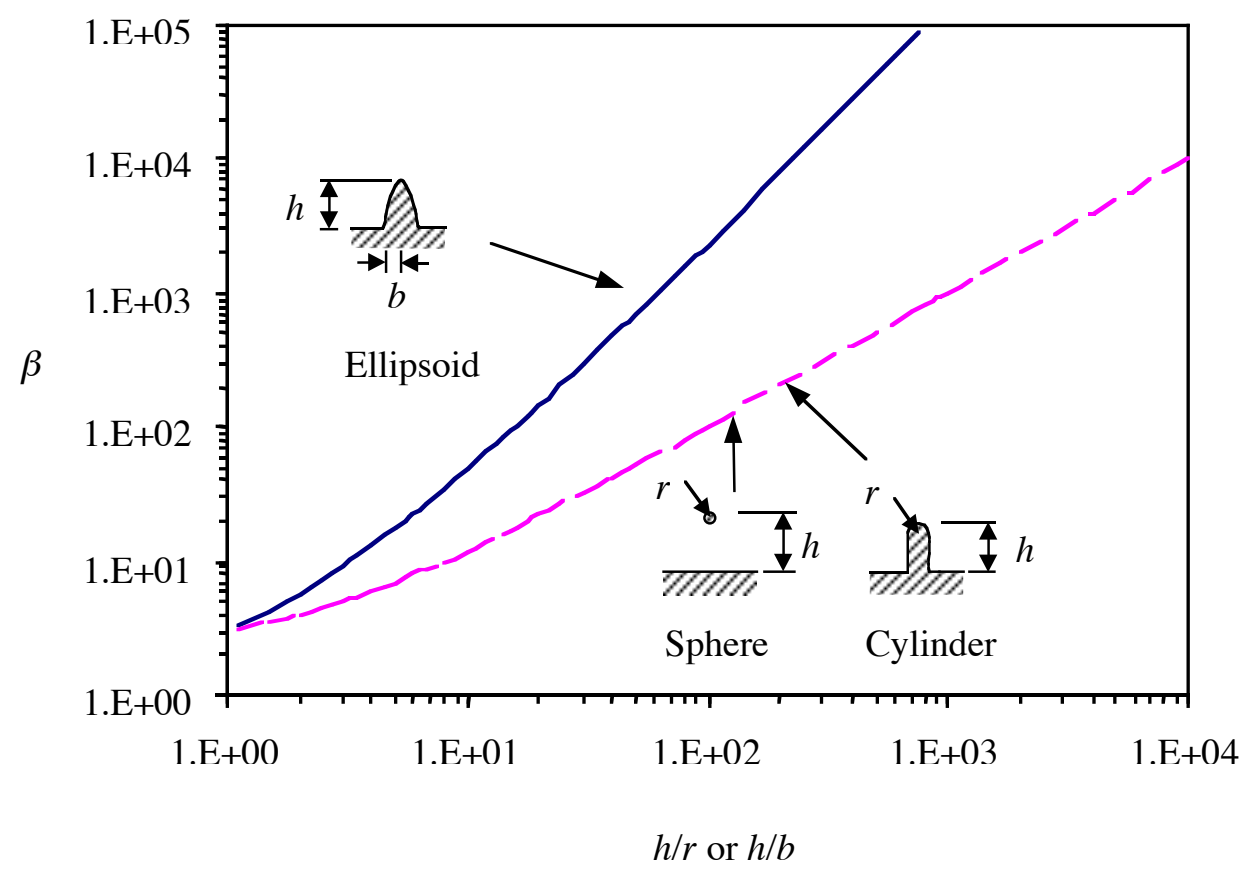

Figure 2.8: Geometrical enhancement factor $\beta$ calculated for various geometries: sphere, cylinder and ellipsoid on top of a flat surface. ${ }^{22}$

-ron shadow microscope, so it is not clearly established that the observed projections were the real emitters. Based on the appearance of the protrusions in milliseconds or less, all long-term surface formations, e.g., room temperature surface diffusion and nucleation effect, are ruled out. One possibility is that the protrusions were already present and rise up as the electric field is applied, which was observed experimentally in DC regime.

III. In Saclay, studies involving intentionally introduced particles of controlled geometry and intentional mechanical damage showed evidence in favor of this projection model: ${ }^{25,26}$ (a) Insulating particles do not emit or emit very weakly while conducting ones usually emit strongly. (b) Spherical metal particles do not emit, even at $120 \mathrm{MV} / \mathrm{m}$, whereas irregularly shaped particles of the same material exhibit strong emission. (c) The oxide layer between particles and substrate does not seem to play an important role in EFE since similar emission behavior was 
observed on $\mathrm{Nb}$ (which has a natural insulating oxide layer) and $\mathrm{Au}$ (which has a conducting surface) substrate and no change in emission property was found even after the metallic particle was welded to and in electrical contact with the substrate after the first emission. (d) The superposed geometrical protrusions may help to explain EFE quantitatively in several types of sites. (Refer to Figure 2.9). (e) Mechanical damage sites emit strongly at low field.

IV. In recent studies at the University of Wuppertal, ${ }^{27}$ they observed emitters with sharp enough geometry to produce the measured $\beta$, although the majority of the fitting parameter $S$ showed unrealistically high values on pure $\mathrm{Nb}$, but tend to become more realistic after the uniform deposition of a $16 \mathrm{~nm}$ gold layer. Moreover, the $\beta$ tends to become smaller after this deposition and this agrees well with the expectation of the projection model.

The Wuppertal calculation showed that the geometrical enhancement factor $\beta$ from the above superposed model is expected to be $\leq \beta_{1} \cdot \beta_{2}$, and can be more accurately expressed as

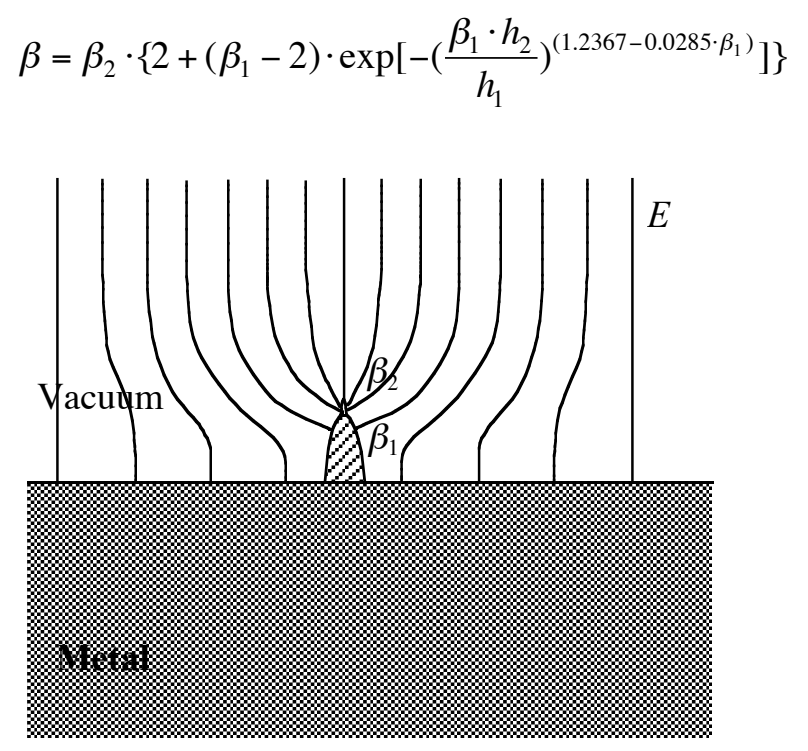

Figure 2.9: The superposed geometrical protrusions to explain the field enhancement factor. 
where $\beta_{1}$ and $\beta_{2}$ are the geometrical enhancement factors for the protrusion and the substructure on top of the protrusion; $h_{1}$ and $h_{2}$ are their heights, respectively.

There are also experimental results against this model:

I. The studies by C. S. Athwal and R. V. Latham et al. ${ }^{28}$ found EFE sites are not generally associated with sharp metallic protrusions, but from 1-2 micron or submicron particles of insulating type (i.e., they appeared charged under an SEM). Surprisingly, $\mathrm{Ph}$. Niedermann did not observe any indication of charging under SEM though the emitters are likely semiconducting or insulating as they always appeared bright under SEM (the secondary electron yield of a semiconductor or insulator is much higher than that of a metal).

II. In their further studies, ${ }^{29}$ energy spectra of emitted electrons were obtained and showed a non-metallic shape, i.e., they were broader than the width of the spectral peak for a metal, and they were shifted from the cathode Fermi level by $0.2 \mathrm{eV}$ or more. Furthermore, the shift and the width of the spectral peak increased with emission current. As a result, they suggested that EFE cannot be a pure metallic process, semiconductors or insulators must play a role instead. Electrons lose energy when they pass through these materials and result in the shift and width change of the spectra. A MIM model, which will be discussed in the following section, was proposed as the underlying cause of EFE.

III. Light emission which is characteristic of electroluminescence in semiconductors was detected from the cathode in many experiments accompanying EFE, as described in previous section. This phenomenon was considered as evidence that the semiconductor was involved in emission.

\subsubsection{Metal-insulator-vacuum model}

The metal-insulation-vacuum (MIV) model was originally suggested by Latham and his co-workers to explain their measured electron energy spectra shift. ${ }^{30,31}$ As illustrated in Figure 2.10, the dielectric properties of the insulator allow the 
complete penetration of the applied electric field into its region, hence producing a highly distorted band structure. Electrons that tunnel through the barrier at the MI interface can accumulate at the bottom of the conduction band of the insulator. Electrons are then accelerated by the internal field and can gain enough energy to overcome the electron affinity barrier to get into vacuum. The model can explain the electron energy shift from the Fermi level by $-\Delta$.

\subsubsection{Insulator switching model}

As described in a previous section, switching has been observed frequently and consistently. A transition (switching) time of $0.2 \mathrm{~ms}$ was reported by Athwal and Latham. ${ }^{32}$

This model was developed from the basis of the MIV model, as described above, to explain this switching phenomenon. ${ }^{33,34}$ In this model, the MI interface initially blocks the electron current in the "switched-off" state, with band structure similar to that as shown in Figure 2.10, i.e., the internal electric field within the insulator is approximately uniform. When the electric field increases, a positive charge builds up

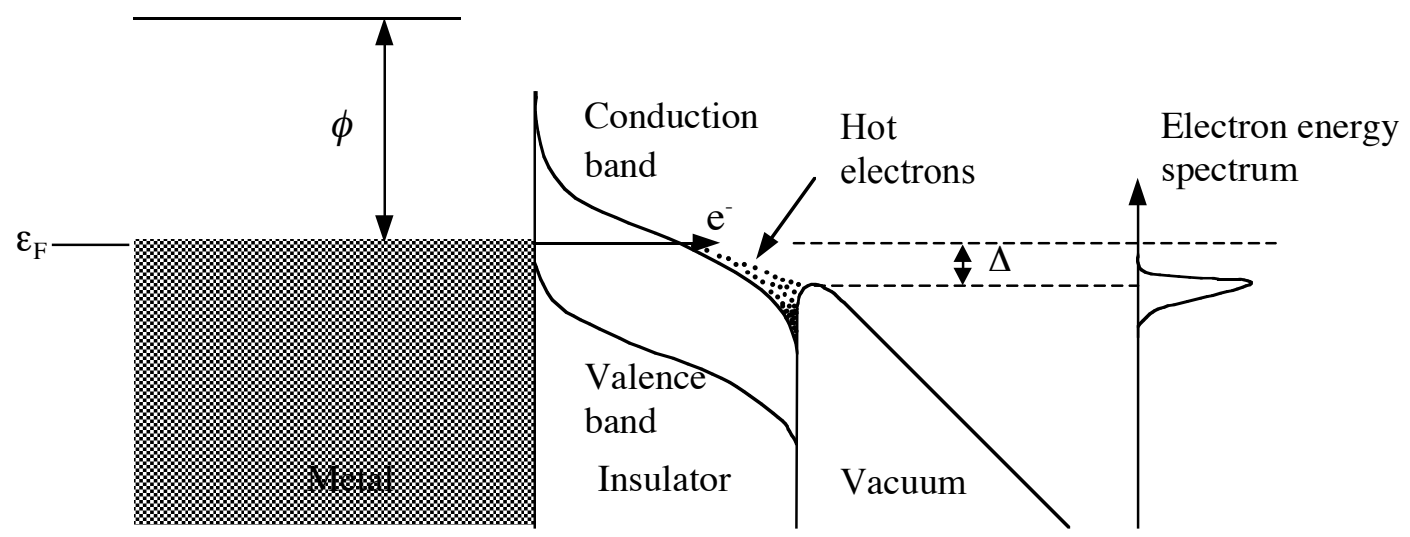

Figure 2.10: Band diagram for MIV model. 
at the MI interface causes the band bending in the insulator and local field enhancement, and consequently causes the electrons to eject from metal to insulator. Electrons travel in the insulator region in thermal equilibrium and accumulate near the insulator-vacuum interface, thus producing a negative charge build-up at the insulator-vacuum interface, which in turn results in a local high field and leads to a tunneling current. This state is the so-called "switched-on" state. Refer to Figure 2.11 for the band structure of the switched-on state.

There are many possibilities that may be responsible for the initial positive charge build-up at the MI interface as discussed in Ph. Niedermann's thesis. ${ }^{12}$ One would think that this model can be tested by depositing a proper insulating material onto a metal surface, but no such experiments have been done successfully up to now. If this model is correct, one would also expect that a deposition of metal film on emitters would induce a strong decrease of $\beta$, which is contrary to T. Habermann's observations. ${ }^{27}$

An important requisite for this model is the initial blocking of charge as the external field is applied to the surface. This is evidenced by the charging appearance of emitting carbon (pencil deposit) particles in C. S. Athwal's experiments, though

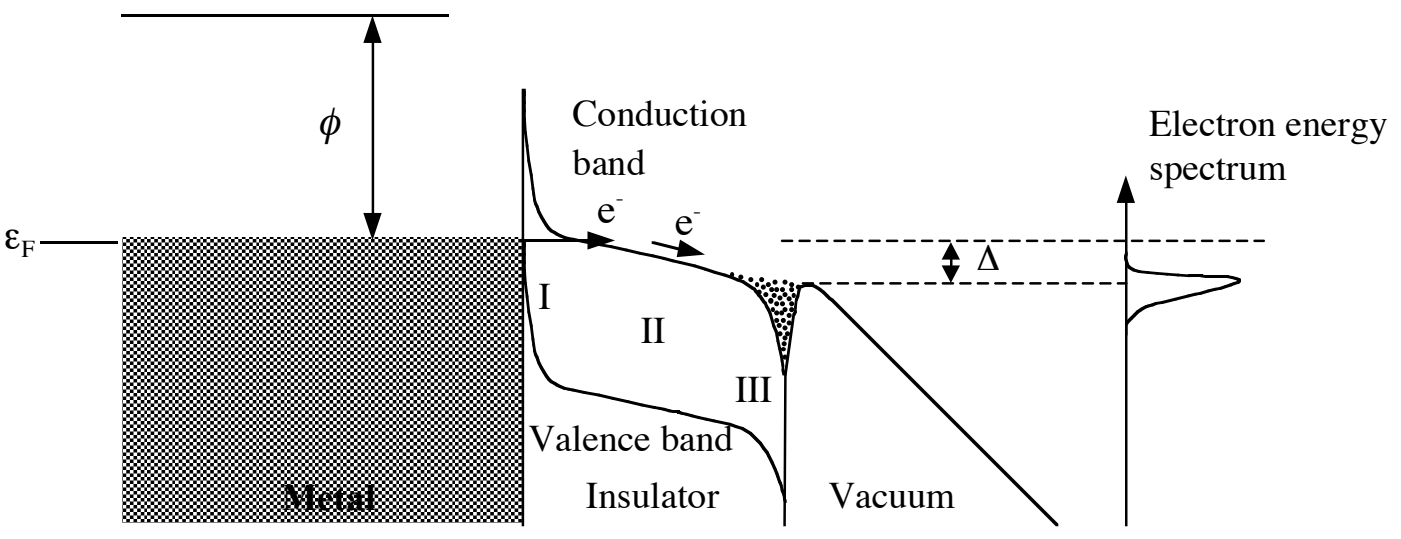

Figure 2.11: Band diagram for a metal-insulator system involving band bending due to the accumulation of space charges with applied electric field. 
in Niedermann's studies no charging except for some $\mathrm{MoS}_{2}$ particles was observed on emitters.

\subsubsection{Metal-insulator-metal model}

MIM model, sometimes called antenna model, was proposed to explain the shift in the electron energy spectra. ${ }^{29,35}$ In this model, a metal flake is present on top of the insulating layer. Since it is not grounded, the flake will act as an antenna probing the electric potential and will adopt the potential close to the equipotential at the flake's highest point, as shown in Figure 2.12. If the height of the flake is $h$, then at the insulator region the field is enhanced by a factor on the order of $h / d$, with $d$ as the thickness of the insulator.

\subsubsection{Filament model}

This model was proposed by Hurley as a result of his electroluminescent measurement, as described in a previous section. ${ }^{19,36}$ This model assumes a certain

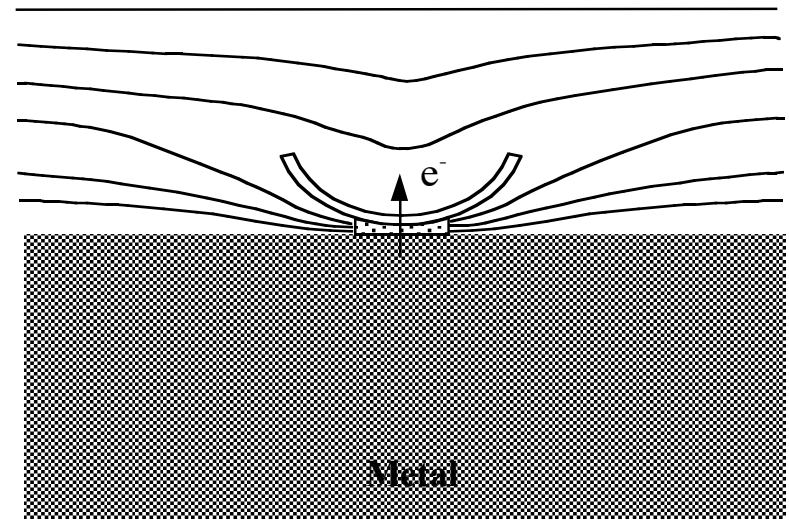

Figure 2.12: The MIM model (antenna model), with a thin conducting flake on top of an insulating layer. The equipotential lines of the electric field are schematically drawn. 
thin layer of semiconducting or insulating impurity on top of the metal, for instance, oxide, nitride, fluoride, etc., which is well-known to be able to transform from a nonconducting to a conducting state with the application of strong external electric fields $(10-100 \mathrm{MV} / \mathrm{m})$, and is accompanied by field electron emission and electroluminescence. ${ }^{37,38}$ The underlying mechanism proposed involves the forming of a conducting channel or filament in the non-conducting impurity, at cracks or grain boundary areas where the local field within the insulator region is enhanced by a geometrical factor $\beta$ to satisfy the electrical field requirement of $10-100 \mathrm{MV} / \mathrm{m}$ for the switching to occur. As a result, the filament will behave like a metallic whisker and promote field emission at the oxide-vacuum interface. Refer to Figure 2.13 for the model illustration.

\subsubsection{Resonant tunneling model}

This model proposes that an unstable surface state results in adatom resonant tunneling, ${ }^{31}$ as shown in Figure 2.14. Calculations by C. B. Duke and others show that electrons with energy close to that of the localized states, for instance, created by surface adsorbate/condensation, can be resonantly enhanced in the tunneling process. ${ }^{39,40}$ Although there is a significant amount of evidence that adsorbed gas is involved in field emission, this model alone, however, can not explain the high field enhancement factor.

\subsubsection{Thermionic emission model}

The model was proposed without involving quantum-mechanical tunneling by Latham. ${ }^{41}$ As illustrated in Figure 2.15, electrons can transmit past a very thin tunnel barrier from metal to the insulator region and are heated by the electric field in the insulator. Electrons with high energy can pass over the surface potential barrier in a thermionic process. The Richardson-Dushman thermionic emission law is assumed to be valid: 


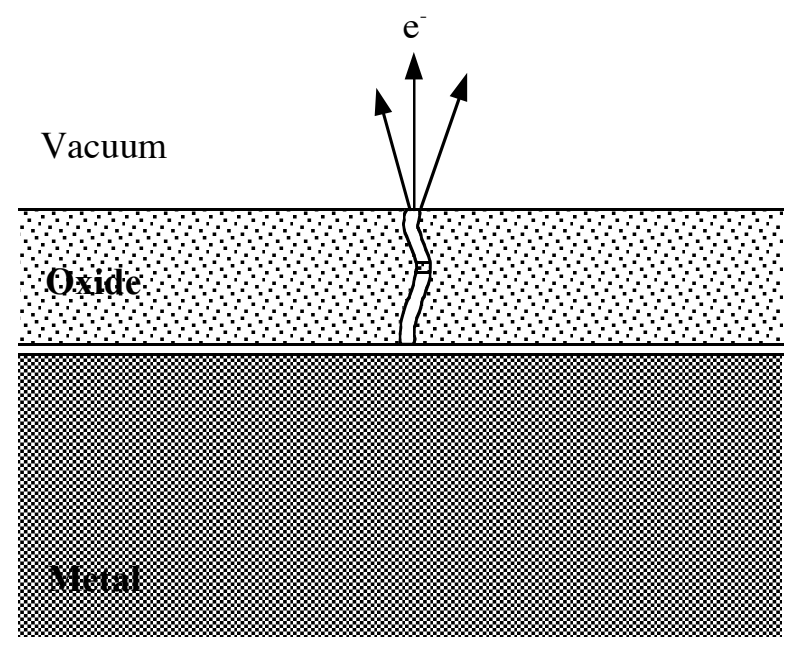

Figure 2.13: Filament model proposed by Hurley, involving a certain semiconducting or insulating thin layer on top of metal. ${ }^{36}$

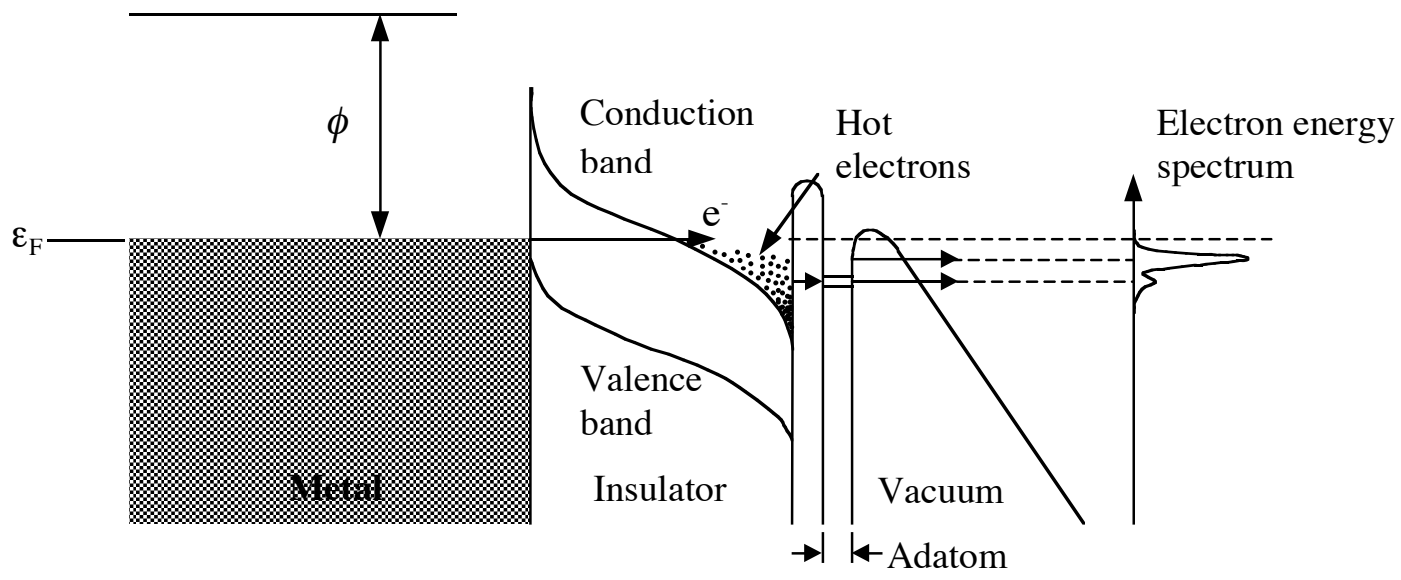

Figure 2.14: Resonant tunneling model: a local energy level is created due to adsorbate on top of a metal-insulator interface. ${ }^{31}$ 


$$
j=K \cdot T_{e}^{2} \cdot \exp \left\{-\frac{e \chi}{k T_{e}}\right\}
$$

where $\chi$ is the electron affinity of the insulator, $K$ is the constant for the RichardsonDushman law $\left(=1.2 \times 10^{6} \mathrm{Amp} /\left(\mathrm{m}^{2} \mathrm{~K}^{2}\right)\right), T_{\mathrm{e}}$ is the electron temperature, assumed to be given essentially by the voltage drop in the insulator, i.e., $T_{\mathrm{e}}=2 \mathrm{e} \cdot V / 3 \mathrm{k}$. The voltage drop $V$ is $\cong d \cdot E / \varepsilon_{\mathrm{r}}$, where $E$ is the electric field, $d$ is the insulator thickness and $\varepsilon_{\mathrm{r}}$ is the dielectric constant. By substituting $T_{\mathrm{e}}$ into Eq. 2.14, the current $I$ can be expressed as

$$
I=K \cdot S^{\prime} \cdot\left(\frac{2 e d}{3 k \varepsilon_{r}}\right)^{2} \cdot E^{2} \cdot \exp \left\{-\frac{3 \chi \varepsilon_{r}}{2 d E}\right\}
$$

where $S^{\prime}$ is the emitting area. The field enhancement factor $\beta$ from F-N law here takes a new significance:

$$
\beta \cong 3.6 \cdot 10^{10} \cdot d /\left(\chi \varepsilon_{r}\right),
$$

where $d$ is in meters and $\chi$ is in V. Although the $I-E$ relation also indicates linearity in the F-N plot, this model has a thickness requirement of $\geq 0.1 \mu \mathrm{m}$ in order to reach $\beta$ of over 300 , which is difficult for the insulator to meet. ${ }^{12}$

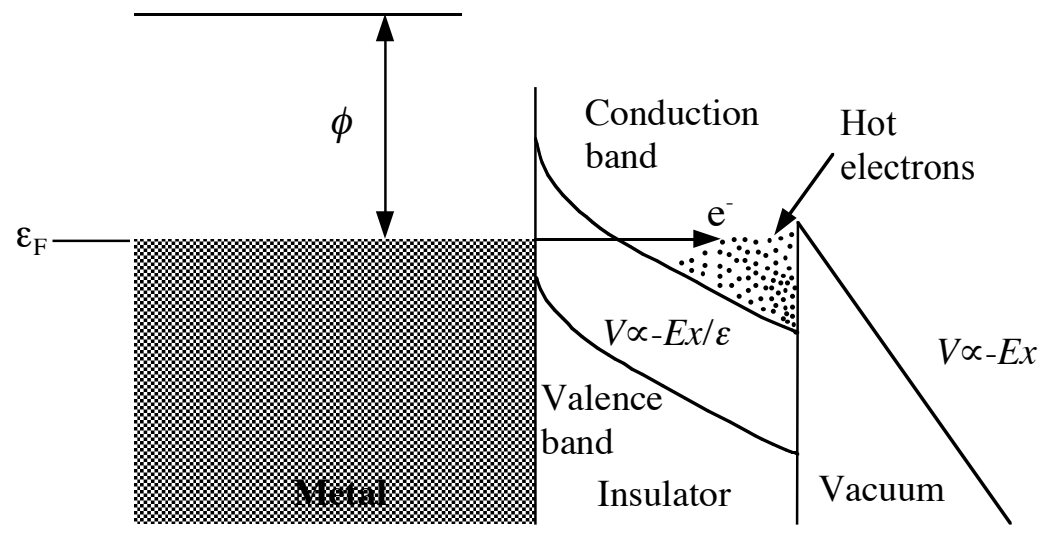

Figure 2.15: Thermionic emission in a metal-insulator-vacuum system. ${ }^{41}$ 


\subsubsection{Discussion}

The comparison between the projection model and emitter geometry is equivocal because of the resolution limit of SEM and the geometrical complication in realistic emitters. In addition, it is illustrated that the modified F-N equation is not valid when applied to sharp emitters ( $r \leq 10 \mathrm{~nm}$, where $r$ is the curvature radius) and will lead to spurious results when extracting information such as field values or emitting area from experimental F-N curves. ${ }^{42}$

As described above, inconsistent results have been reported, especially on the conducting or insulating properties of emitters. The insulating or semiconducting type energy spectra needs to be investigated further, since it is not clear if the insulating composition is the reason or critical for EFE or just present as an unavoidable contaminant due to the reaction between the sample and residual gas. Generally, this inconsistency is possibly related to different preparation and cleaning procedures and/or sample handling in different environments. Therefore, detailed investigations on carefully prepared, thoroughly cleaned $\mathrm{Nb}$ samples is of importance for resolving the ambiguity. 


\section{Chapter 3}

\section{Summary of previous research results on enhanced field emission from niobium}

Prior field emission work was mentioned in the previous chapter during the discussion of the physical mechanisms of field emission. We dedicate this chapter to the experimental aspects of some of the work directly related to $\mathrm{Nb}$ in the $\mathrm{DC}$ or $\mathrm{RF}$ regimes.

Overview of enhanced RF field emission measurements have been given by Noer and Weingarten. ${ }^{14,43}$ The measured current-electric field characteristic agrees well with the F-N law (equation 2.4) integrated over an RF period

$$
I \propto E^{2.5} \cdot \exp \left\{-\frac{B \cdot \phi^{\frac{3}{2}} \cdot v(y)}{\beta \cdot E}\right\}
$$

where $E$ is the magnitude of the local electric surface field. It should be noted that in SRF cavities, the surface electric field peaks at iris areas, therefore field emission should and usually does predominantly occur in this region, where $E=E_{\mathrm{pk}}$. A RF field emission measurement was done by Hübner with a tungsten micropoint emitter inserted in an RF resonator, and the results agree well with equation (3.1). ${ }^{44}$ Since no general difference between RF and DC field emission is expected, ${ }^{45} \mathrm{DC}$ as well as RF apparatus have been built in several labs, including University of Geneva, Cornell University, Centre d'Etudes de Saclay (Saclay) and Institut de Physique Nucleaire (IPN-Orsay) and University of Wuppertal, and their results show general 
agreement on the categories of emitters. ${ }^{12,46,47,48}$ Advantages of DC apparatus include more accurate location of emitters and in situ observation and characterization of emitters.

\subsection{Results from University of Geneva}

In the early 1980s, Ph. Niedermann at the University of Geneva set up an ultra high vacuum (base pressure of $5 \times 10^{-11} \mathrm{mbar}$ ) DC field emission scanning apparatus, using the surface analysis system "Escalab" from Vacuum Generators, which is equipped with an electron gun, secondary electron detector, and Auger analyzer for in situ analysis of emitters. A sample stage, anode tips and anode holder were added to the "Escalab" chamber for FE scanning. ${ }^{12}$ This system was equipped with an electron gun of $0.5 \mu \mathrm{m}$ beam diameter, a $157^{\circ}$ spherical sector electron analyzer with its input lens system for Auger Electron Spectrometer, secondary electron detector and argon ion gun. The sample was moved in a raster pattern under the anode at $E$ up to $100 \mathrm{MV} / \mathrm{m}$ with the field emission current detection threshold $I$ set at $\approx 40$ nA. The scan area was $12 \mathrm{~mm} \times 12 \mathrm{~mm}$. The detected FE sites were then locally scanned with high resolution using a microtip anode at a gap of 20-50 $\mu \mathrm{m}$, with the resolution in locating emitters determined mainly by the gap.

In summary of their findings, FE sites were generally associated with micron or submicron sized particles sitting probably rather loosely on the surface. The particles mostly contain a metal: $\mathrm{Nb}, \mathrm{Al}$ or $\mathrm{Ag}$, sometimes also a high carbon concentration hundreds of angstroms deep. They also observed an effect of heat treatment at different temperatures, i.e., annealing at up to $900^{\circ} \mathrm{C}$ increases the density of emitter sites, while annealing at $1200^{\circ} \mathrm{C}$ or higher reduced the number of emitting sites significantly. The phenomenon remained true with repeated heating cycles. Although each individual sample showed considerably different absolute value of emitting sites from initial condition through the heating cycle with no correlation to initial preparation process, they all follow the general trend with the 


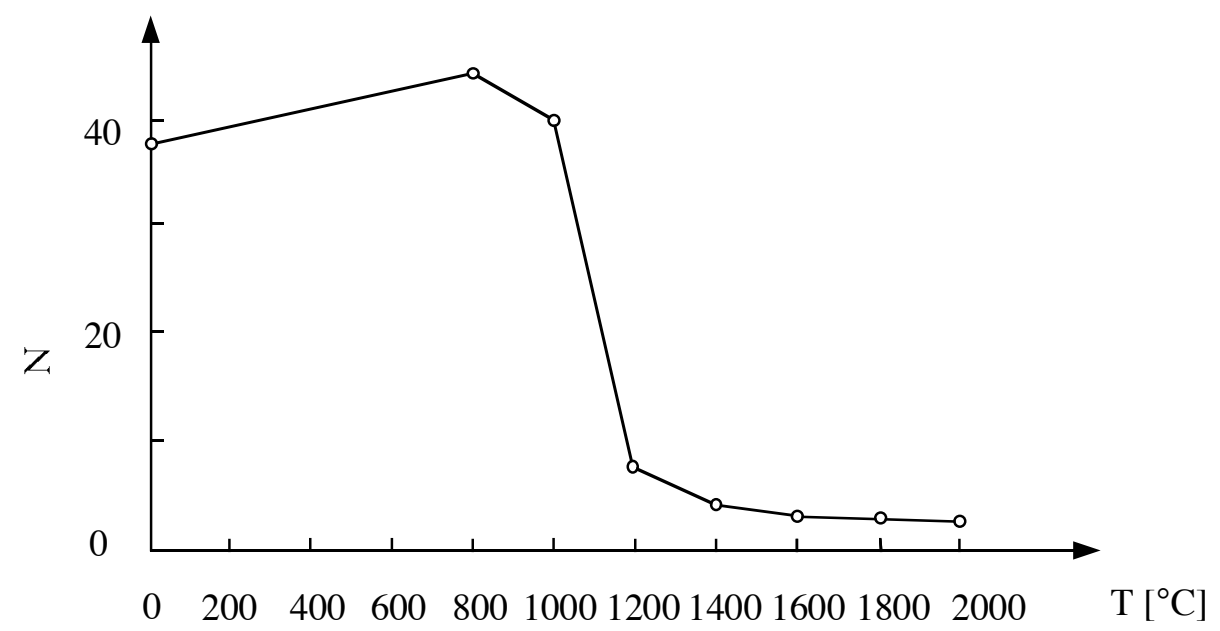

Figure 3.1: Average number of emitting sites per sample through a heating cycle at $90 \mathrm{MV} / \mathrm{m}^{12}$

annealing temperature. Refer to Figure 3.1 for the average number of emitting sites per sample through a heating cycle up to $2000^{\circ} \mathrm{C}$.

They achieved emission free surfaces on several niobium samples at $100 \mathrm{MV} / \mathrm{m}$ by heat treatment at $1400^{\circ} \mathrm{C}$ or higher. The microscopy and elemental analysis on emitters indicated that:

I. Particles are usually found at new emitting sites that appeared after medium temperature heat treatment. The particles can be divided into three categories: those containing sulfur; those containing carbon; and those containing other foreign elements, for instance, Ag, W, Cu, Ca, Si, Cr, or Mn. Particles containing sulfur only appeared after the heating, while particles containing carbon were already present at the beginning, but did not emit before the heating. Many of the foreign elements can be speculated to be related to the material used or maybe present in their vacuum chamber, for example, silver paste, $\mathrm{MoS}_{2}$ lubricant, dust specks $\left(\mathrm{CaCO}_{3}\right)$. 
II. Heat treatment at high temperature often changed the shape, brightness or elemental composition of particles, which may be related to their change in field emission behavior.

\subsection{Results from Saclay and Orsay}

B. Bonin and colleagues made use of a conventional SEM equipped with EDS by adding an anode probe in the chamber to scan the $\mathrm{Nb}$ sample at up to $200 \mathrm{MV} / \mathrm{m} .{ }^{49}$ The working pressure in the SEM is $1 \times 10^{-6}$ Torr. All FE sites were confirmed to be correlated with two kinds of defects: geometrical defects (no foreign elements detected by EDS), and particulate contamination. ${ }^{50}$ However, not all defects emit and no clear criteria were identified for those that did. The following is a list of chemical composition found in the foreign particles (emitting and non-emitting): O, C, Si, Al, Ca, K, Na, Cl, Fe, Mg, F, Ti, Zn, N, Ag, Ni, Cr, etc. In order to overcome the difficulty of research on natural emitters due to the unstable emission and the tendency of being blown away under high electric field, they carefully studied artificial particulate contamination and found that most of the conducting particles (Fe, $\mathrm{Ni}, \mathrm{Au}, \mathrm{Nb}, \mathrm{Ti}$ ) tend to emit at low field levels, while most insulating ones $\left(\mathrm{Al}_{2} \mathrm{O}_{3}, \mathrm{SiO}_{2}\right)$ do not emit even at high fields. Also the morphology of the particles influences emission, spherical particles do not emit but irregular ones emit strongly.

\subsection{Results from Cornell University}

D. Moffat and colleagues at Cornell University developed a mushroom cavity, in which a dimple is exposed to very high RF electric field, as well as three S-band cavities which have been subjected to high electric field and then cut open for SEM examination $^{48}$. They located the FE sites on the surface according to certain features that are usually found on FE sites intentionally created by placing carbon flakes ( 200 $\mu$ m diameter), a reliable FE source, on the Nb cavity surface. The features 


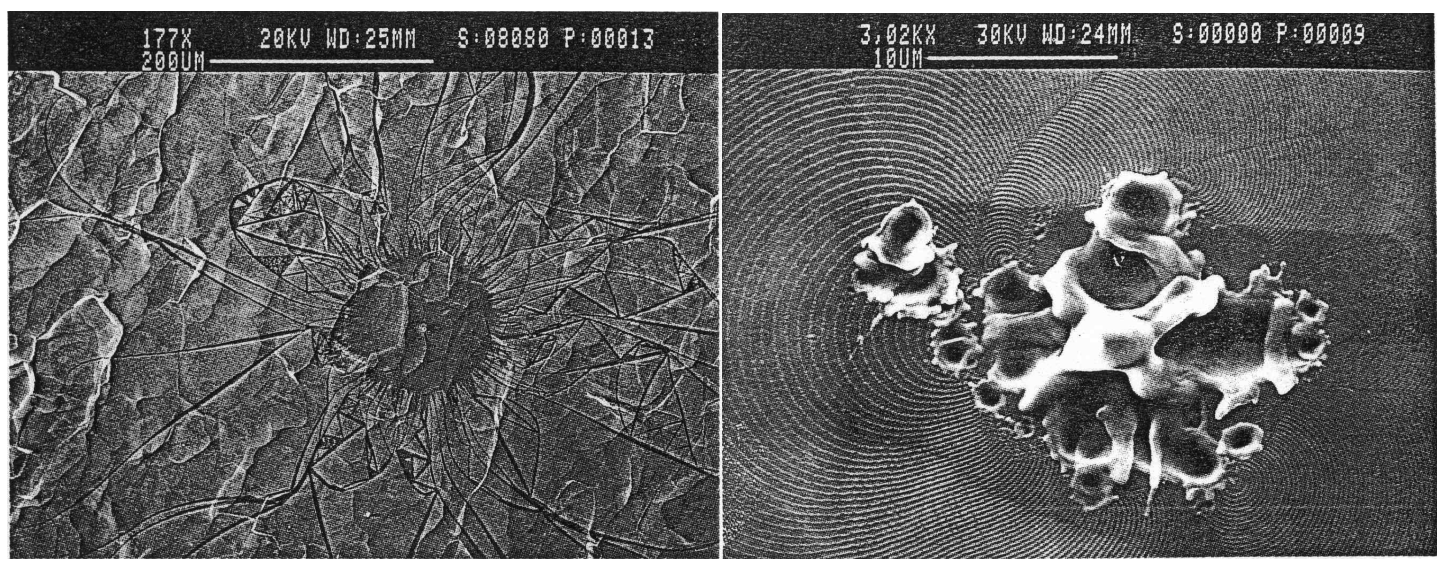

Figure 3.2: Features found at field emission sites in SRF cavities, showing starburst and tracking (left) and craters and ripples (right).

include: starburst, tracking, craters of molten $\mathrm{Nb}$, ripple pattern and/or particles of foreign elements. Refer to Figure 3.2 for pictures of such features.

No foreign elements or increase in the concentration of oxygen or carbon were found in the starburst or craters (by EDS), or in the ripples or the spaces between them (by scanning Auger Microscope). Approximately half or less of the starbursts contain foreign particle(s) near its center according to EDS analysis, while the foreign elements detected include Fe/stainless steel, In, $\mathrm{Cu}, \mathrm{Ti}$, Teflon and other traces of elements, like $\mathrm{Na}, \mathrm{Mg}, \mathrm{Al}, \mathrm{Cl}, \mathrm{Ca}$. Impurities were found, however, at every emission site of the mushroom cavity through AES, and the starburst corresponded to a depleted fluorine area. It was estimated that the fluorine layer is $50-1500$ angstrom deep at surrounding surface based on two argon sputterings of the surface. The fact that the impurities, including stainless steel, $\mathrm{Si}, \mathrm{Ni}, \mathrm{Mn}, \mathrm{In}, \mathrm{Cu}, \mathrm{C}, \mathrm{F}$ or $\mathrm{Cl}$, are present in all FE sites supports the idea that impurity particles are responsible for FE. Of course, it is possible that other elements exist on the surface since particles of light element composition may vaporize completely during FE. In many cases, the enhancement mechanism could persist several FE melting cycles (each 
successively formed crater is offset from the previous one, forming a path of overlapped craters).

During SRF cavity testing, two techniques can be used to process field emitters: high peak power pulse processing and helium processing. High peak power pulse processing increases the applied field to a sufficiently high value for a very brief time, usually $\approx 5 \mu \mathrm{sec}$, so that the local surface field at the emitter $\beta E$, and hence the emitting current, is high enough to cause the emitter to melt. As a result, field emitters can be destroyed or processed away in situ. Helium processing lets in a controlled amount of helium gas to the cavities, with helium gas to enhance the production of plasma at the emitting sites. This permits the processing of emitters at lower fields than would ordinarily be possible. ${ }^{1}$

At Cornell, a DC apparatus was also developed for FE studies. The apparatus consists of two electrodes in a vacuum chamber. The cathode is a thin plate with $25.4 \mathrm{~mm}$ diameter having five raised pedestals, each $1 \mathrm{~mm}^{2}$ in size. The anode has an effective area of $1 \mathrm{~mm}^{2}$ and can be moved laterally to center under a particular pedestal. Artificial particles were placed on the pedestals to induce vacuum breakdowns at high electric field. Then the cathode was removed from the vacuum chamber and examined under SEM at the sparking or emitting sites. Starburst and craters of molten $\mathrm{Nb}$ were observed only at the sites that sparked, whereas no trace was left at sites that did not spark even though they emitted much higher currents.

\subsection{Results from University of Wuppertal}

Following the example of the University of Geneva, E. Mahner, G. Muller and coworkers at the University of Wuppertal built a similar apparatus using "Escalab" in the early 1990s. Their results also show that EFE sources are localized micron or submicron sites, and that many sites appeared to be particles or geometrical scratches/damages, and that most emitters contain foreign elements although the types of elements differ. ${ }^{46}$ 
They designed and built a Field Emission Scanning Microscope, similar to the University of Geneva's type, in the same VG "Escalab" UHV analysis system with the addition of a preparation chamber. ${ }^{51}$ The system is equipped with a SEM (resolution $70 \mathrm{~nm}$ ), an external long distance microscope (from Questar, $1.8 \mu \mathrm{m}$ resolution at a working distance of $23 \mathrm{~cm}$ ) for optical control of the gap between sample and anode tips, an AES for element detection, and a micro-focus ion sputter gun (spot size $\approx 30 \mu \mathrm{m})$. In the preparation chamber, samples can be heat treated up to $2000^{\circ} \mathrm{C}$ by electron bombardment in a base pressure of $1 \times 10^{-9} \mathrm{mbar}$. They employed a special electronic circuit to rapidly lower the anode voltage by a fast high voltage regulator and a shunt tube when FE current is detected during the sample scanning in a raster or chequered pattern, in order to hold the emission current at a constant value $I_{0}$, typically set at $10 \mathrm{nA}$. Therefore the resulting high voltage variation $\Delta U$ on anode and $I_{0}$ characterize the $\mathrm{FE}$ strength on a specific emitting site. $x, y, z$ motions are driven by microprocessor-controlled stepper motors and the resolution and reproducibility of the displacement were measured with the SEM to be approximately $0.2 \mu \mathrm{m}$ and $5 \mu \mathrm{m}$, respectively.

From their first results on non-heat-treated samples, all localized FE sites were associated with micron-sized particles, mechanical defects like microscratches, and etch pits from chemical etching. Microdischarge and sparks can create new emitting sites. ${ }^{52}$ Also observed is that a large number of particles present on the surface do not emit up to $100 \mathrm{MV} / \mathrm{m}^{53}$ Their results also showed that heat treatment at $400-900^{\circ} \mathrm{C}$ increased the average emission strength of emitters and stimulated new field emitters, then a subsequent heat treatment at higher temperature $1200-1400^{\circ} \mathrm{C}$ will switch off most of them. The phenomenon still holds true after repeated heat treatment cycles.

In SEM studies of all localized sites, isolated particles with random shape were found. The analyzed sites contain $\mathrm{Al}, \mathrm{W}, \mathrm{Ca}, \mathrm{Ti}, \mathrm{Cu}, \mathrm{S}, \mathrm{C}, \mathrm{O}, \mathrm{Nb}, \mathrm{Si}, \mathrm{Cs}$ and an enhanced concentration of $\mathrm{C}$ or $\mathrm{O}$ was found in $50 \%$ of the sites. It is interesting to 
note that none of the localized emitters in the first heat treatment cycle was identified as a site in the second cycle. They believe that the $600-800^{\circ} \mathrm{C}$ heat treatment activate the emission of particles that already exist on the sample but do not emit in the first cycle. This activation could be associated with surface diffusion or segregation of interstitial atoms from or into the bulk $\mathrm{Nb}$ because the density of activated particles was about seven times larger for $\mathrm{Nb}$ of $\mathrm{RRR}=30$ compared to $\mathrm{Nb}$ with $\mathrm{RRR}=1000,{ }^{13}$ as shown in Figure 3.3. This suggestion is evidenced by segregation experiment after mild heating in UHV system. ${ }^{54-57}$ It has been observed that sulfur segregates on many transition metals surfaces during 600-900 C heating and is removed after heating to $1000^{\circ} \mathrm{C}$ or higher. Other experiments showed that oxygen segregation occurred on $\mathrm{Nb}$ surface after heating at $300^{\circ} \mathrm{C}$ under ideal $\mathrm{UHV}$ condition. $^{58}$

From Auger analysis on broad area Nb surfaces after heating at low temperature, the oxygen/niobium ratio is strongly reduced, and the carbon/niobium ratio shows a smaller reduction and carbide sub-peaks occurred. Traces of sulfur were detected after heating between $200-800^{\circ} \mathrm{C}$, most pronounced on the purest $\mathrm{Nb}$. There is no other significant surface diffusion or segregation of interstitial atoms except $\mathrm{C}, \mathrm{O}$ and S. They also proposed explanations for the $400^{\circ} \mathrm{C}$-effect: change of chemical composition of particle (e.g., $\mathrm{Nb}_{2} \mathrm{O}_{5} \Rightarrow \mathrm{NbO}$ ), or change of conductivity of contact between particle and $\mathrm{Nb}$ surface that create an emitting MIM-structure.

They also have successfully reduced FE particles by high pressure ultrapure water rinsing (HPWR) combined with final methanol rinsing (MR) with no heat treatment, but other types of surface irregularities like scratches with traces of metal (e.g., Fe, Cr, and Ni) become EFE sites. ${ }^{46}$ Reduced or zero emission was achieved on $\mathrm{Nb}$ samples of $\mathrm{cm}^{2}$ size at $100 \mathrm{MV} / \mathrm{m}$ by $\mathrm{BCP}$ and water rinse. No information is reported on the reproducibility of this performance. Their most recently reported statistics of emitter density from niobium after various surface preparation are illustrated in Figure 3.4. 


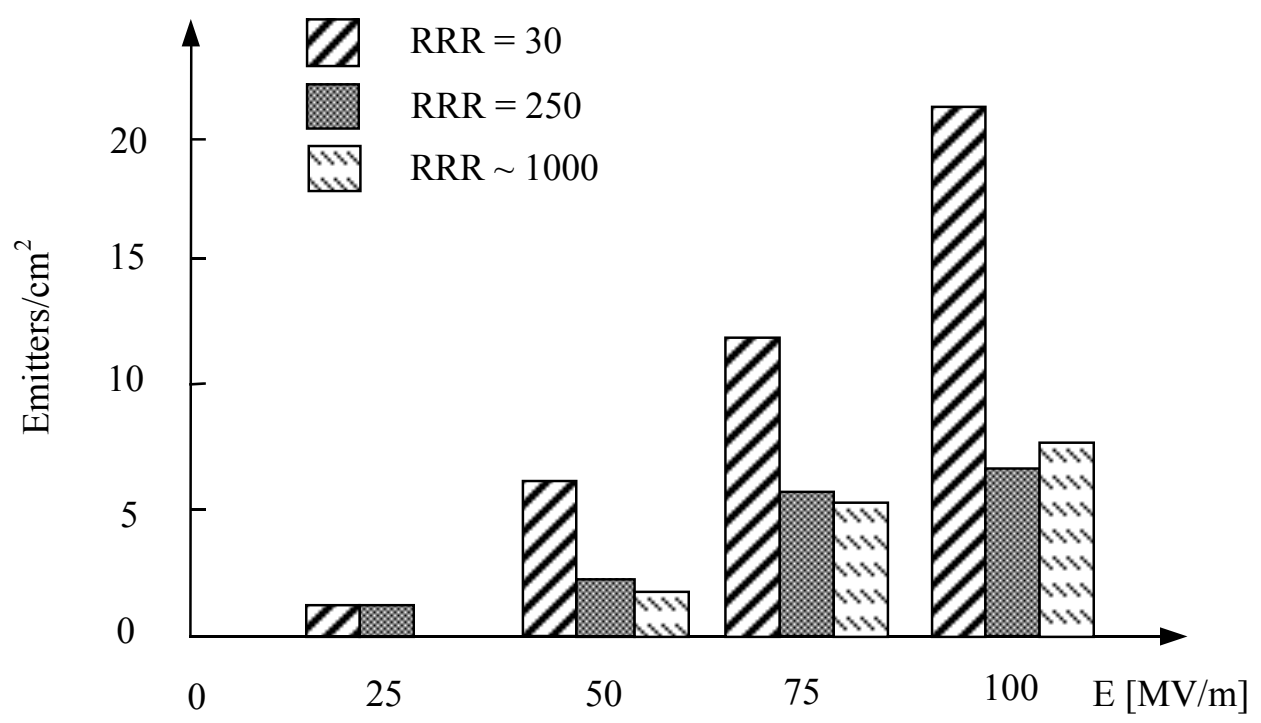

Figure 3.3: Emitting sites density for various purity of niobium, the higher the RRR, the purer the material. All localized emitting sites were particles containing $\mathrm{Nb}$ and Fe. (6 samples, BCP 1:1:1 removal of $\sim 170 \mu \mathrm{m}$, high pressure ( 80 bar) ultra-pure (17.5 $\mathrm{M} \Omega \mathrm{cm})$ water rinsing, electronic grade methanol rinsing).

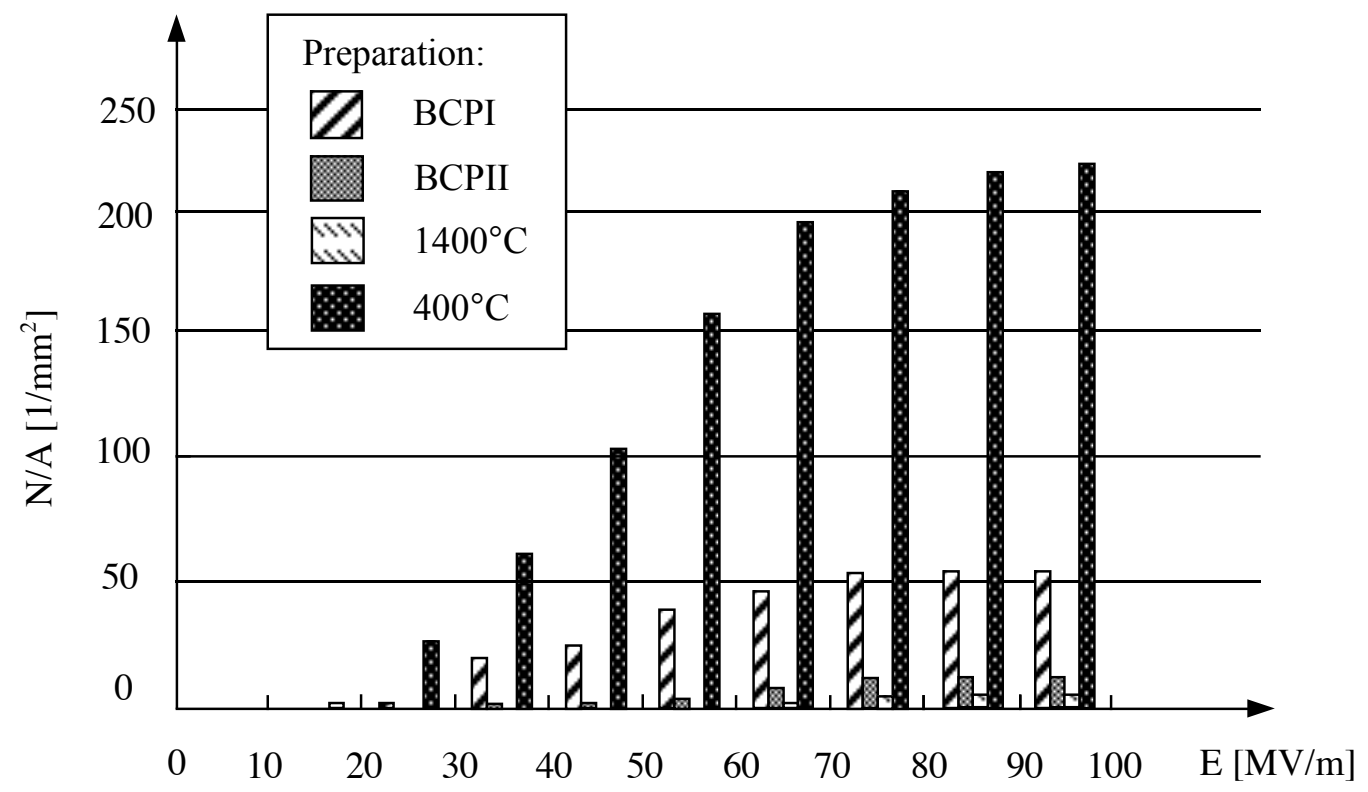

Figure 3.4: Statistics of emitter density reported by the University of Wuppertal. 


\subsection{Summary}

In review of some of the findings, a few qualitative conclusions are reached:

(1) Naturally occurring EFE can not be completely attributed to geometrical field enhancement.

(2) Most of the emitters observed are associated with contamination with metallic particles, but need to be activated by some unknown condition of the cathode surface.

(3) Surface irregularities can become emitters if triggered by traces of embedded impurities.

The University of Geneva's FE work ended in the late 1980s, and the University of Wuppertal is focusing on diamond film and discontinuing high field work with $\mathrm{Nb}$. This thesis project was proposed in order to continue FE work on contemporary $\mathrm{Nb}$ surfaces. 


\section{Chapter 4}

\section{Experimental program, apparatus and procedures}

\subsection{Experimental program}

\subsubsection{Experimental objectives}

The mechanism for enhanced field emission remained unclear at the beginning of this work. The bulk of studied emission sites are most probably external instead of intrinsic due to the different, non-standard preparation and handling procedures and, especially, the unavailability of High Pressure Water Rinsing (HPWR) in earlier studies. Hence we proposed a systematic study of FE sources from planar Nb with respect to various preparation techniques. The experimental objectives are:

I. To design and build an ultra high vacuum DC field emission scanning apparatus capable of locating individual emitters on $\mathrm{Nb}$ and of providing in situ microscopic characterization.

II. To conduct a systematic study of field emission sources with respect to the best available cleaning techniques, including chemical etching by BCP (refer to chapter 5), electropolishing (refer to chapter 6), HPWR (refer to chapter 7), and possibly heat treatment if necessary. 
a) For each category of emitters, to identify the technique(s) that reliably removes them to the point of their elimination, or to a point which yields a clear characterization as to where additional effort would be needed. To establish a sequence of techniques to minimize field emission, as this will leave emitter sources that are more intrinsic to the material and are ready for further studies. Only through this approach can we overcome the reproducibility problems encountered previously in field emission studies of the performance from $\mathrm{Nb}$.

b) To devise a methodology to distinguish external particulate emitters from intrinsic impurities as this will provide information on the directions in which effort is needed for their removal. (The definition of external emitters and intrinsic emitters will be given in the following paragraph.)

c) To gain some information and understanding in regards to the physical mechanism of enhanced field emission from their microscopic appearances and elemental compositions.

External emitters, a term that when used in this thesis refers to geometrical damage (scratches, etc.) and foreign particles coming from handling and machining, have been evidenced by past experimental results. Intrinsic emitters, a term that when used in this thesis refers to grain boundary and material bulk impurity, were suggested to be possible emitters because of the local field enhancement factor and/or evidence from early experimental work, ${ }^{59}$ but have not been experimentally confirmed or refuted for naturally occurring emitters on high purity contemporary metal surfaces. The categories of emitters on material surface are schematically illustrated in Figure 4.1. True F-N field emission, which occurs at above the GV/m level, and is sometimes also referred to as intrinsic emission, should not be confused with the intrinsic emission definition in this thesis, i.e., for this work all FE refers to EFE unless noted otherwise. 
Dust particle

Geometrical damage from machining and handling

Foreign material inclusion from machining

Chemical residue

Grain boundary

Bulk impurity or segregation

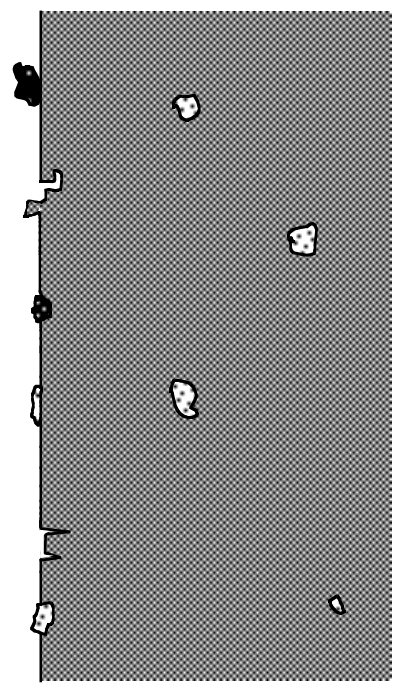

Figure 4.1: A schematic illustration of known and potential emitters on material surface.

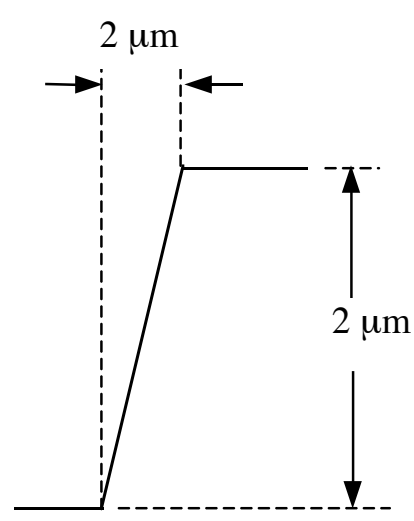

Chemically Polished
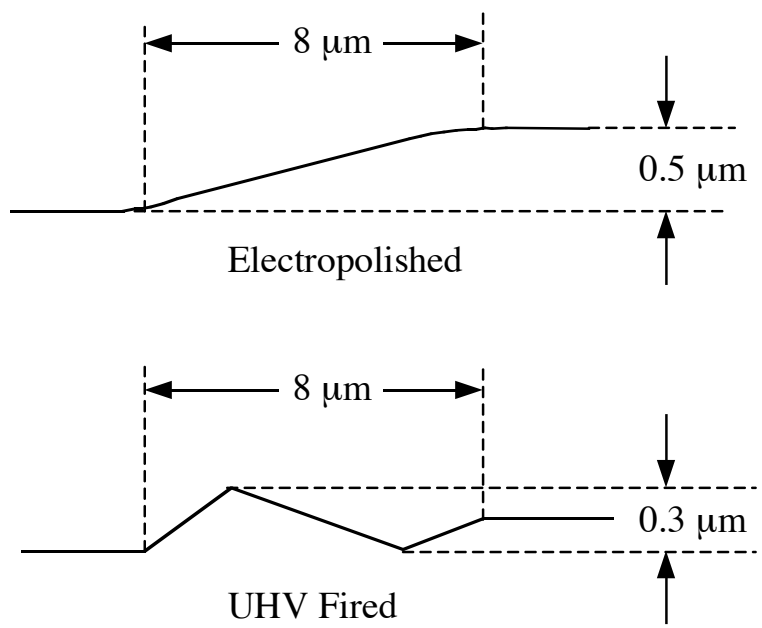

Figure 4.2: Grain boundary geometry from chemically etched (by BCP), electropolished and UHV fired (heat treated, $\sim 2100^{\circ} \mathrm{C}$ ) $\mathrm{Nb}$ surfaces. 


\subsubsection{Experimental plan}

Following the route of the most easily removed, most frequently encountered to the less frequently encountered emitter type, we proposed a systematic program for the removal of all four categories of emitters (refer to Figure 4.3). To help demonstrate the underlying reasons for the sequence in the program, the grain boundary geometry measured by Sundelin from chemically etched, electropolished, and UHV fired $\mathrm{Nb}$ surface is shown in Figure $4.2 .^{60}$ As one would note that electropolishing yields the smoothest grain boundary, followed by UHV firing, and chemical etching produces the sharpest geometry at grain boundaries.

Chemical etching by BCP (buffered chemical polishing) followed by UWR (ultrasonic water rinse) would be a good point to start, and the amount of removal needed to eliminate most of the machining damage and contaminants and whether etch pits are emitters are to be investigated. It is highly likely that this step will get rid of most of the big and loosely attached particles. A powerful tool to clean external particles-HPWR (high pressure water rinse) would be the next step to further clean the smaller or tightly attached particles. The operating parameters of HPWR can be optimized in this step for maximum removal of particles. However, there still could be a small number of particles left due to their size or possibly being stuck at a grain boundary. Thus, EP (electropolishing) will be an ideal process to follow up to flatten the grain boundary, a potential emitter itself, and in the mean time to make it less likely for the particles to be stuck in a grain boundary and resist being washed away. Nonetheless, there still might be a few tiny particles or bulk impurities that emit after all the processes above, and HT (heat treatment at $1400^{\circ} \mathrm{C}$ ) will likely decompose and/or evaporate them. If particles are welded to the surface due to HT and remain or become emitters, further $\mathrm{BCP}$ or $\mathrm{EP}$ is needed to get rid of them. 


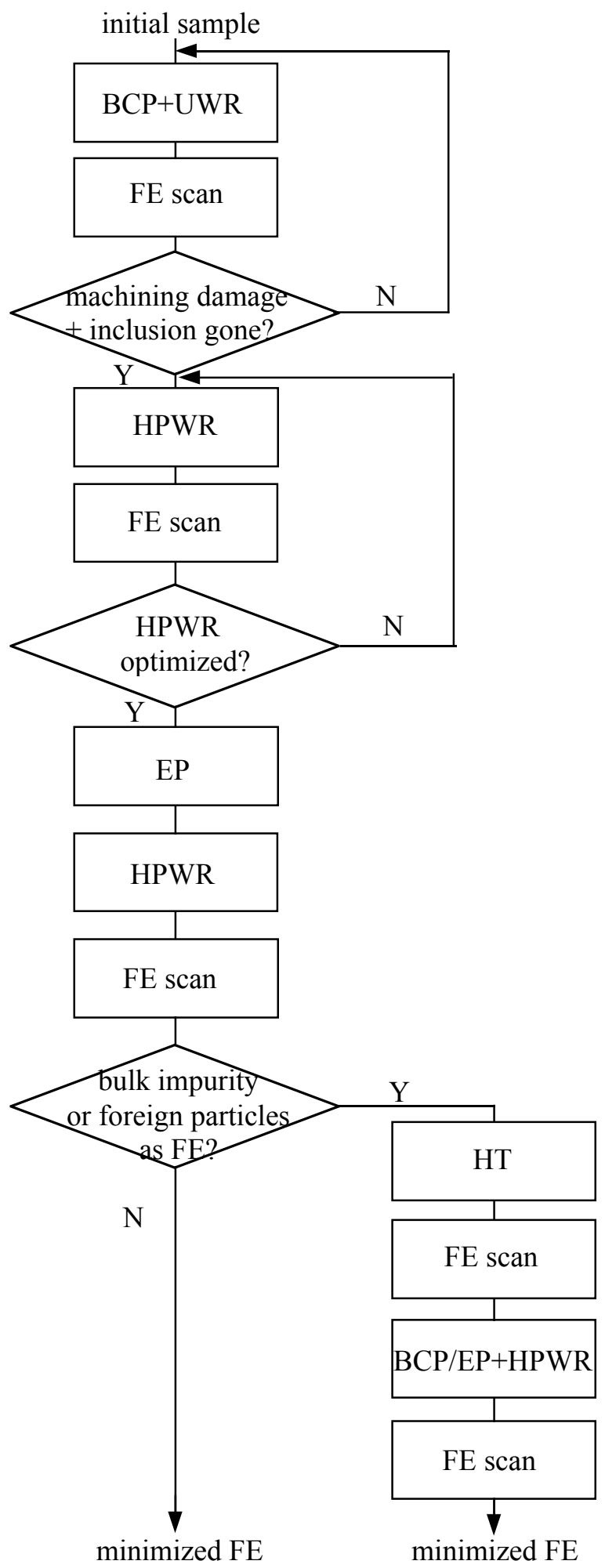

Figure 4.3: Program to categorically remove field emitters. (BCP: buffered chemical polishing, UWR: ultrasonic water rinse, HPWR: high pressure water rinse, EP: electropolishing, $\mathrm{HT}$ : heat treatment at high temperature $\left(\sim 1400^{\circ} \mathrm{C}\right)$. 
In the process described above, after optimized HPWR to remove the majority of foreign particles, information on whether intrinsic emitters, i.e., grain boundary and bulk impurity, exist can be obtained using a methodology, which will be described later in this chapter.

\subsection{Experimental apparatus}

\subsubsection{Ultra high vacuum chambers design}

We designed and built an apparatus, termed a Scanning Field Emission Microscope (SFEM). It is a UHV device ( $10^{-9}$ Torr), attached through a UHV bellow and a gate valve to an Amray Scanning Electron Microscope (SEM 1830) with a nominal resolution of several nm, as shown in Figure 4.4. A heat treatment (HT) chamber is attached to the SFEM by a gate valve. The SFEM and HT chambers each have a dedicated ion pump and use the turbo and mechanical pumps of the SEM system for roughing. Samples are loaded via the SEM, and can be transferred under vacuum to the other two chambers by a hermetic retractable linear rotary transporter (travel range: $914 \mathrm{~mm}$ ). The SFEM and HT chambers are supported by a vibration-isolated frame. The UHV bellow between the SFEM and SEM provides vibration isolation between the two chambers.

Three viewports are installed on the SFEM chamber, one for observation during sample loading and unloading, one for monitoring through an optical microscope, and one for providing illumination. The long distance optical microscope is mounted above the SFEM chamber for observation and recording of the sample surface and for monitoring gap consistency during field emission scan through a CCD camera and a TV monitor. A frame grabber card installed on the PC can grab and digitize the image for storage. The long distance microscope from Questar (model: QM100, working range: $15-35 \mathrm{~cm}$ ) has a nominal resolution of $1.1 \mu \mathrm{m}$ at a working distance of $15 \mathrm{~cm}$. The position of the microscope relative to sample and anode tip is illustrated in Figure 4.5. 


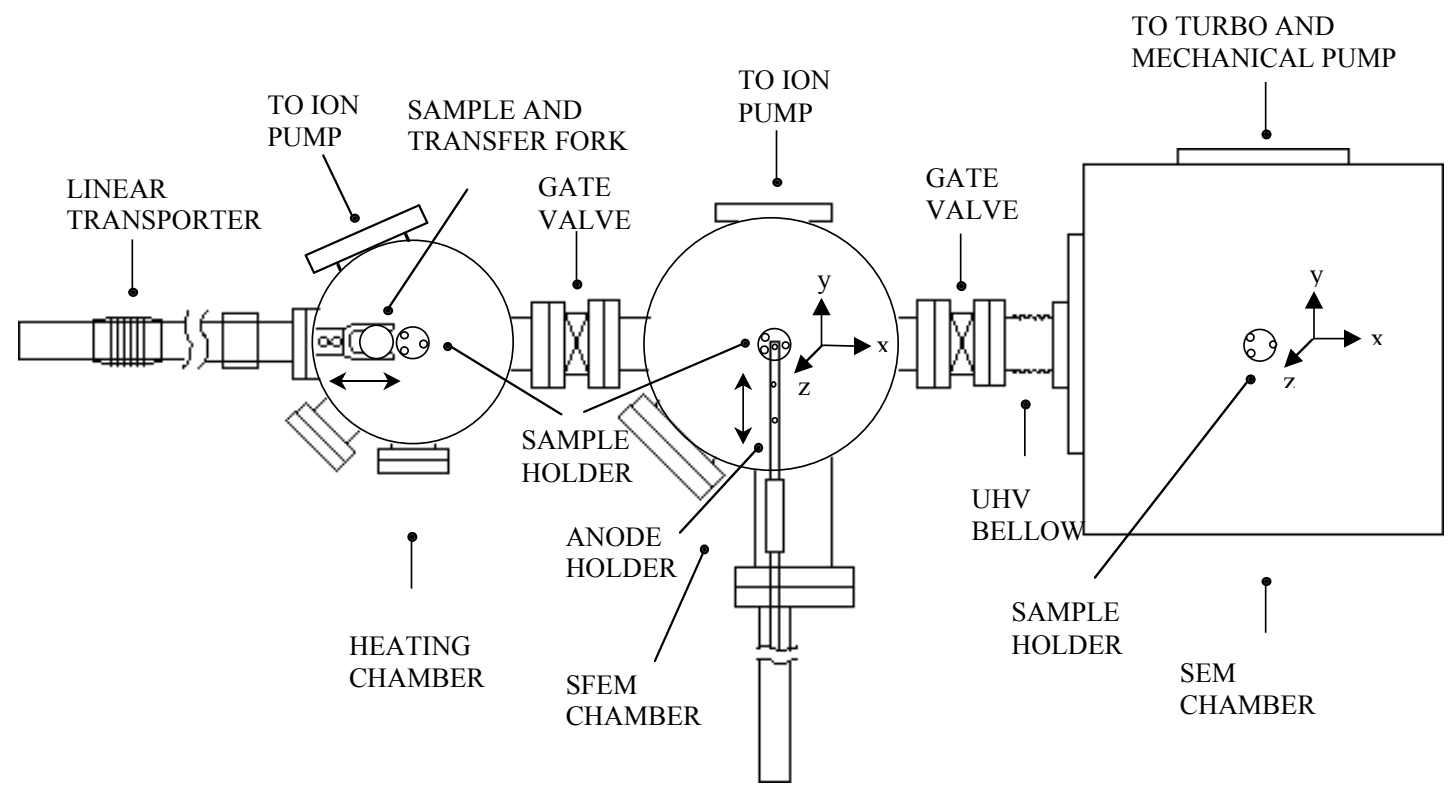

Figure 4.4: Top view of experimental apparatus.

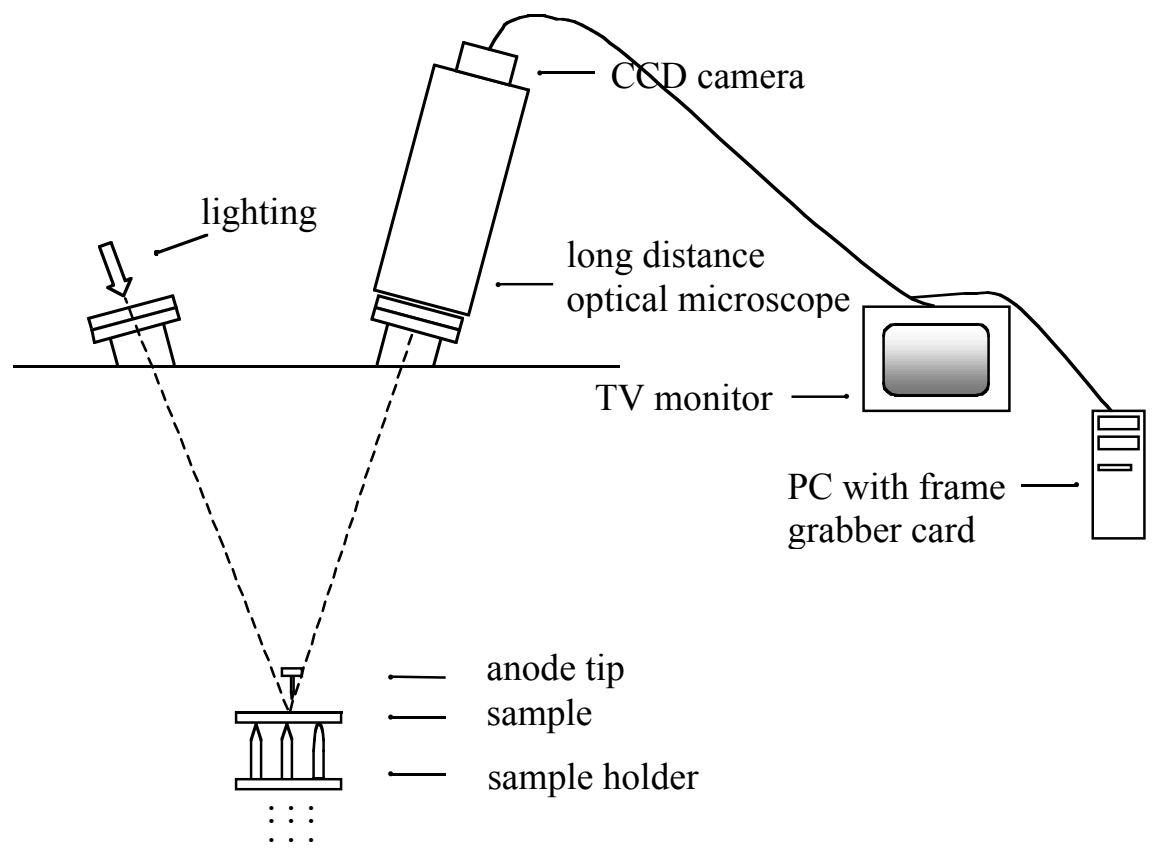

Figure 4.5: The position of the long distance optical microscope relative to sample and anode tip in SFEM chamber. 
Within the SFEM chamber, samples of slightly larger than $25 \mathrm{~mm}$ diameter can be moved in $x, y$, and $z$ under an anode tip by a motorized high precision sample manipulator. Anode tips are mounted on a high voltage anode holder that can be moved linearly for tip exchange. Tungsten anodes of 150, 10, and $1 \mu \mathrm{m}$ tip radius can be selected for coarse, medium, and fine scans. After emitters are located in the SFEM chamber, the sample is transferred to the SEM chamber for emitter characterization. The SEM is equipped with EDS (Energy Dispersive Spectrometer) capable of windowless operation for light element sensitivity.

Three artificial marks on each sample surface are used as fiducials to calculate the $x, y$ coordinates of emitters in the SEM chamber from their coordinates in the SFEM chamber, as illustrated in Figure 4.6. The common accuracy in re-locating emitters under SEM is $\pm 100-200 \mu \mathrm{m}$. As the sample preparation process is progressively refined and controlled, the number of emitters per sample has dropped to $1-2 /$ sample, as will be addressed in following chapters. With the low number of emitters, and the fact that the $\pm 500 \mu \mathrm{m}$ area around the calculated emitter location is always closely examined for any micron or submicron scale features under the SEM at 320x magnification, the identification of emitters is routinely unambiguous, and can often be further confirmed by a second FE scan after an ultrasonic water rinse to remove the suspected emitting particle.

Understandably, the built-in accuracy of the apparatus in relocating emitters is not as good as those combining FE scan with microscopy analysis in the same chamber, e.g., the apparatus in University of Geneva and that in University of Wuppertal, however, this apparatus design was chosen due to considerations on reducing cost, enhancing flexibility of the operation (not to interfere with the routine operation of the SEM by other users), and taking advantage of the high resolution of the existing commercial SEM, which is better than the apparatus in Geneva and Wuppertal. 

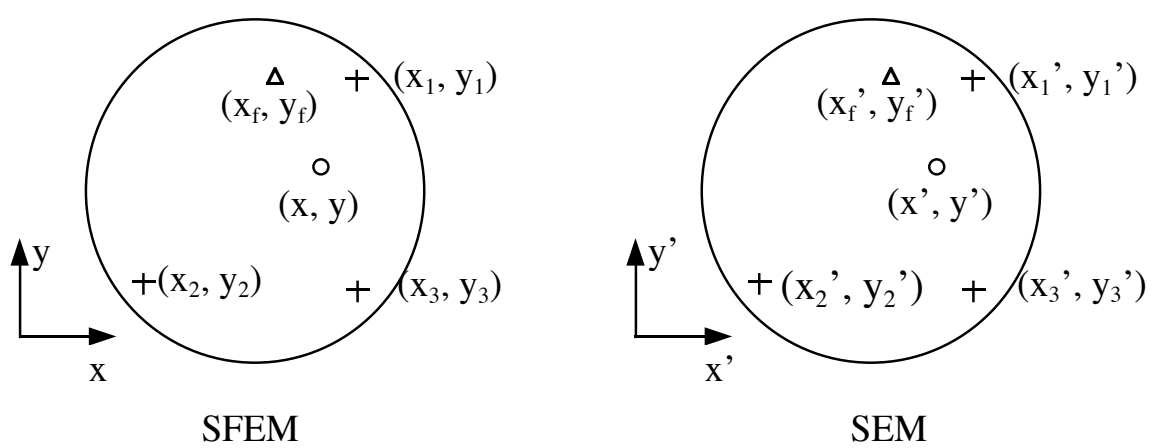

"+": artificial surface marks

"o": emitters

" $\Delta$ ": features seen from SEM

known: $\quad\left(x_{1}, y_{1}\right),\left(x_{1}{ }^{\prime}, y_{1}{ }^{\prime}\right),\left(x_{2}, y_{2}\right),\left(x_{2}, y_{2}{ }^{\prime}\right),\left(x_{3}, y_{3}\right),\left(x_{3}, y_{3}{ }^{\prime}\right)$

therefore: $\quad(\mathrm{x}, \mathrm{y}) \quad \Rightarrow\left(\mathrm{x}^{\prime}, \mathrm{y}^{\prime}\right)$

$\left(\mathrm{x}_{\mathrm{f}}, \mathrm{y}_{\mathrm{f}}\right) \Leftarrow\left(\mathrm{x}_{\mathrm{f}}{ }^{\prime}, \mathrm{y}_{\mathrm{f}}{ }^{\prime}\right)$

Figure 4.6: Three artificial marks on sample surface are used to correlate $x, y$ coordinates in SFEM with $x, y$ coordinates in SEM.

The HT chamber is designed for the purpose of sample outgassing by external radiation and sample thermal processing by electron bombardment up to $1400^{\circ} \mathrm{C}$. Two viewports are installed on HT chamber for observation and external radiation heating respectively (Refer to the last chapter for the reasons for external heating). Emission current for the electron bombardment is designed to reach $0.1 \mathrm{~A}$. The sample is biased at $+6 \mathrm{kV}$ relative to the filament, considering that chamber and other grounded parts inside the chamber can be bombarded if the sample is grounded and filament is biased at $-6 \mathrm{kV}$. The layout is shown in Figure 4.7.

Electrostatic forces acting on the filament in the presence of high voltage tend to distort the filaments and must be guarded against, therefore the filament is supported by six thin $\mathrm{W}$ rods mounted on alumina standoffs to prevent sagging and distortion. A grounded shield made from Mo sheet is mounted around and just 


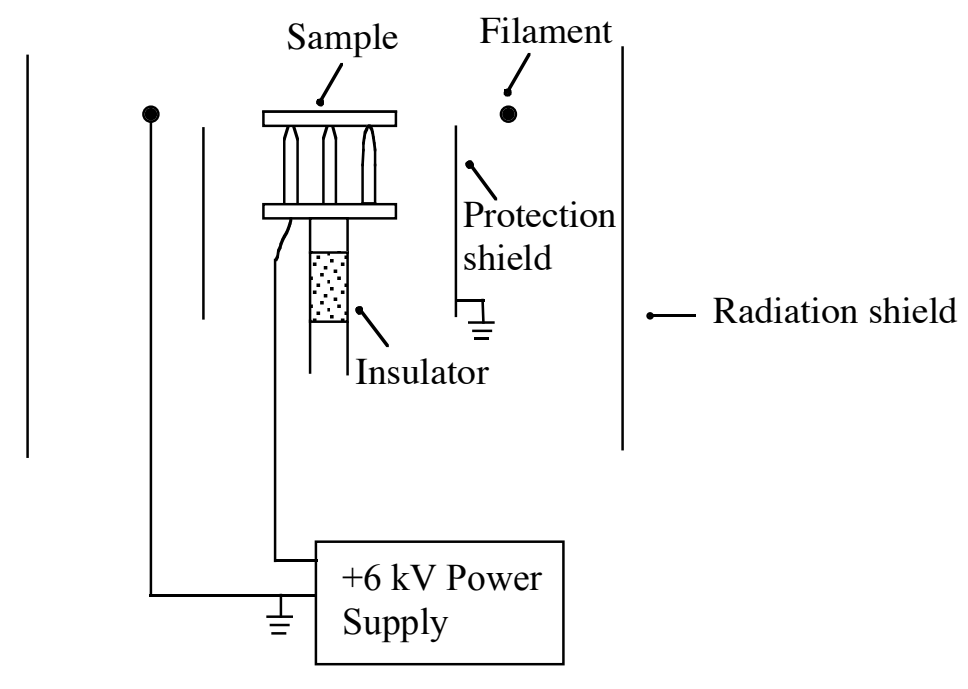

Figure 4.7: Sample heat treatment by electron bombardment.

below the sample to protect sample holder and its alumina standoff from electron bombardment damage.

All filament support structures, power connections and sample holder are made of high temperature material: W or Mo. The Mo radiation shield around the filament assembly is designed to reduce heat loss and prevent potential overheating of critical components in the chamber. The length of the filament power connecting wires, supporting W rods, and the Mo poles of sample holder were all selected by calculating the temperature distribution using MatLab. Joule heating and radiation loss are included in the secondary differential equation.

The temperature of the sample during heat treatment can be monitored by an optical Pyrometer (Capintec, model: KTL-PRO) with a temperature range of 250$2000^{\circ} \mathrm{C}$.

The apparatus is located in a Class 1000 cleanroom to reduce the risk of contamination on the sample's surface during handling. A picture of the apparatus is shown in Figure 4.8. 


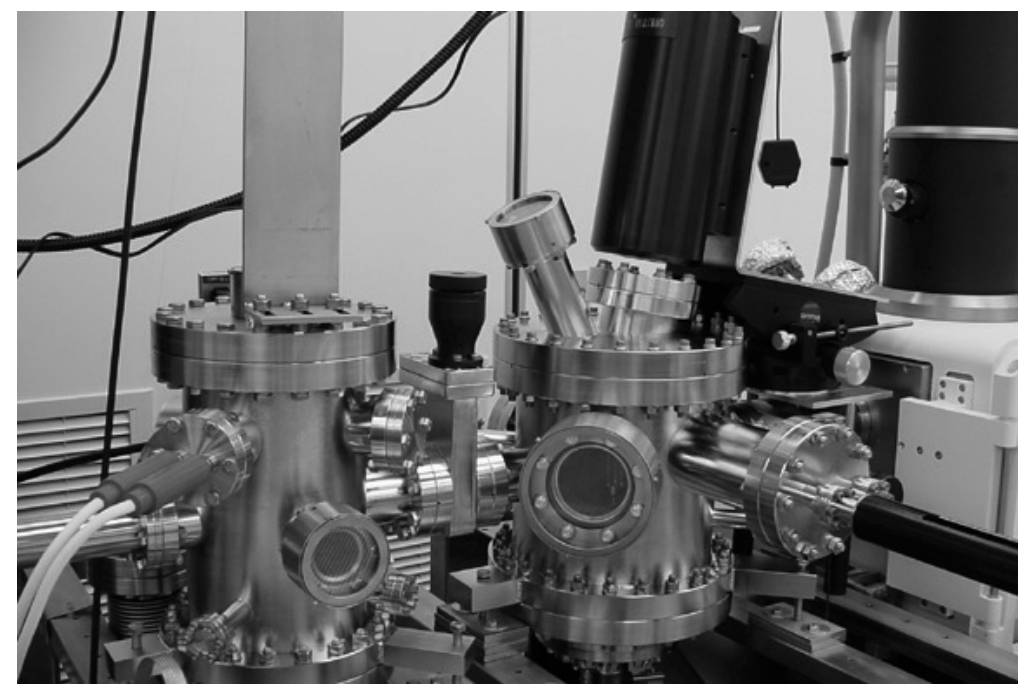

Figure 4.8: Picture of the apparatus.

\subsubsection{Sample and anode manipulation and sample $\mathrm{x}-\mathrm{y}$ plane travel limit switch design}

The motorized UHV sample manipulator (Vacuum Generator, model: HPT-RX) has a step size of $2.5 \mu \mathrm{m}$ in $x, y, z$ and $50 \mathrm{~mm}$ travel range in $z$. Its travel range in $x, y$ plane is a circle of $25 \mathrm{~mm}$ diameter determined by the size of the UHV bellow (35 $\mathrm{mm}$ inner diameter) employed in the manipulator and the size of the sample probe (10 $\mathrm{mm}$ diameter). A circular travel limit device of $35 \mathrm{~mm}$ inner diameter was designed by the author and installed inside the chamber and concentric to the mounting flange of the UHV bellow of the sample manipulator, as illustrated in Figure 4.9 .

The top stainless steel ring of the device is insulated from the rest of the device and the chamber by ceramic, and connected to the Home switch signal $(+5 \mathrm{~V})$ on the stepper motor controller card (Oregon Micro Systems, model: PC34-4). When the sample probe (at ground potential) travels to the limit and touches the top ring, 


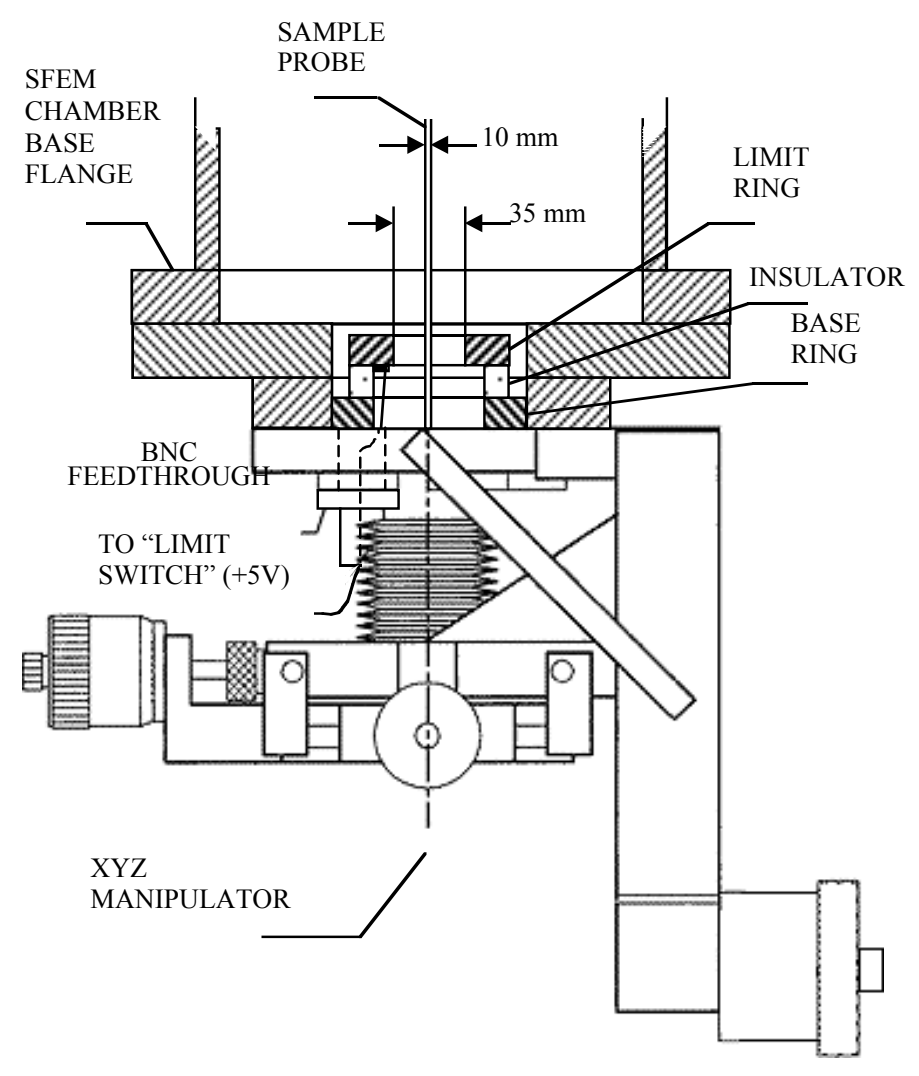

Figure 4.9: The setup of stepper motor travel limit switch.

the "Home" switch is then closed and the signal can be detected by the computer through the controller card. This design avoids potential damage to the manipulator in case of overdrive and enables full utilization of its travel range of $25 \mathrm{~mm}$ diameter, thus providing a larger scan area than the DC FE scanning apparatus built by the University of Geneva $(12 \mathrm{~mm} \times 12 \mathrm{~mm})$ and by the University of Wuppertal ( $\leq 15 \mathrm{~mm}$ diameter). Samples slightly larger than $25 \mathrm{~mm}$ in diameter are used so that the edge area that is vulnerable to machining damage can be avoided during FE scan.

Sample manipulation in $x, y, z$ and anode tip linear exchange (150 mm travel, 2.5 $\mu \mathrm{m}$ step size) are all performed by a computer program written in LabView. The 
LabView driver for the stepper motor control card was developed by the author. The hardware of the control is shown in Figure 4.10.

\subsubsection{Precise sample position registration}

The sample and sample holders in all three chambers, i.e., SEM, SFEM, and HT, are specially designed for the sample to have self-alignment capability in the sample holder, i.e., the sample can return to its previous location and orientation after non-in situ processing. This capability is achieved without a complex mechanical

PC34-4

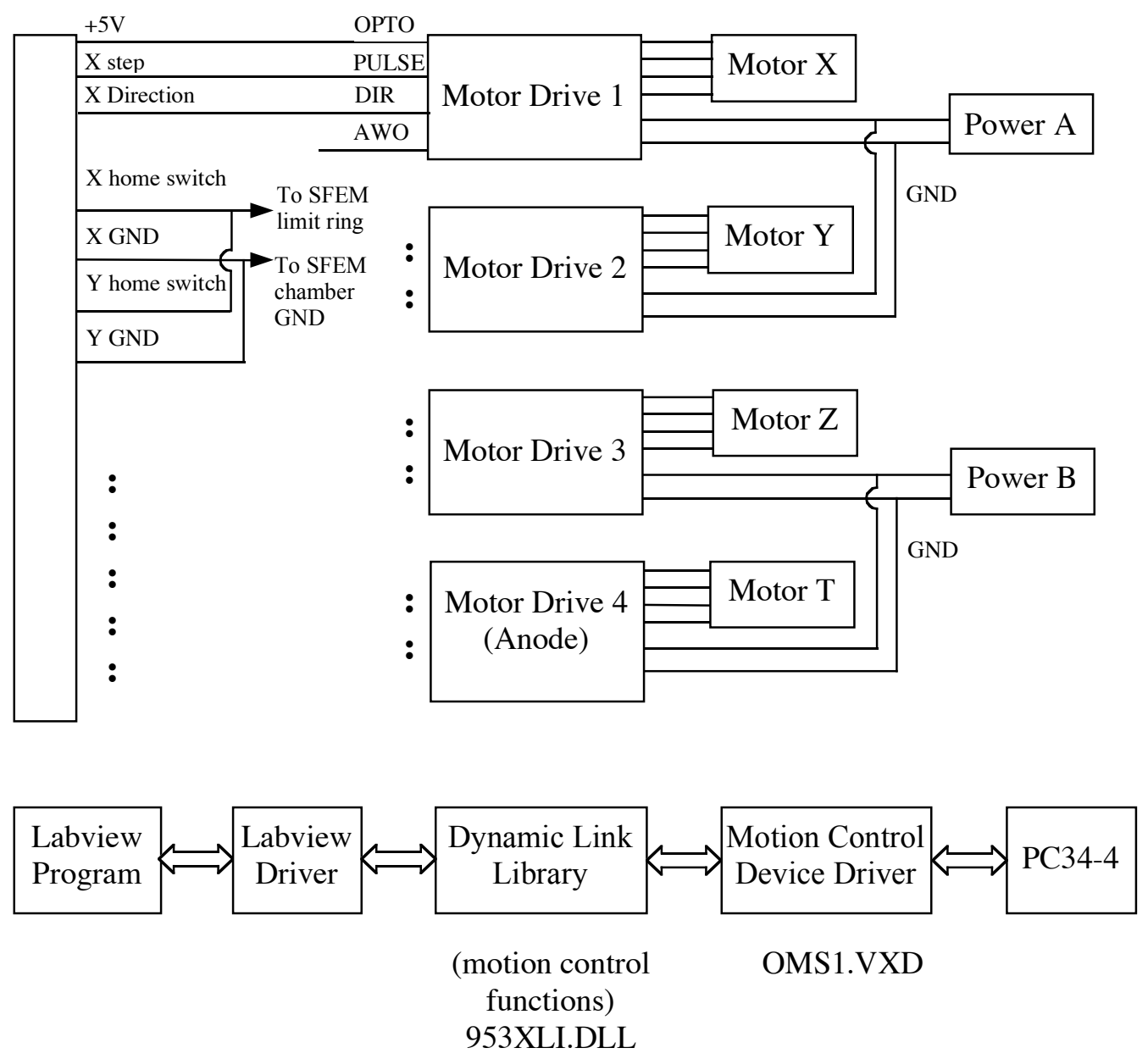

Figure 4.10: The hardware for the stepper motor control. 
fixture and is tolerant of a certain degree of initial misalignment with sample loading. The repeatability in $x, y$ is proven to be $\sim 140 \mu \mathrm{m}$ or better. With the additional aid of surrounding grain shapes as seen with the SEM, interesting micron and submicron features can be tracked through non-in situ as well as in situ preparation. Similarly, the emitting behavior of the same emission sites can also be tracked through in situ or non-in situ processes.

The sample and sample holder design is patent pending and the concept is as follows. The holder has three support posts with two identical conical tips and one hemispherical tip. The sample has one cylindrical hole, one U-groove whose axis intersects the cylindrical hole axis, and one flat area, each of which contacts one of the three support posts. Unambiguous and reproducible sample alignment is accomplished by the simultaneous contact of conical tip (1) with the cylindrical hole, conical tip (2) with the U-groove, and the hemispherical tip with the flat surface on the sample. Hence, all points of the sample are in locations uniquely defined by the sample and sample holder. Highly precise locations of the sample posts, the cylindrical hole, and the U-groove are not required to make the location of a particular sample on a particular sample holder uniquely defined. The only requirements to achieve good alignment are that the contact surfaces be smooth so that the sample can fully seat on the holder posts without being impeded by surface roughness, and that the materials of the sample and sample holder must be such that the coefficient of friction between them does not prevent the sample from fully seating on the holder.

To illustrate the capability of the design to facilitate the relocation of micron to submicron features (and to also provide a way to experimentally distinguish external particles from material impurities after a second water rinse), pictures of a foreign microparticle before and after removal from the apparatus for water rinse are shown in Figure 4.11. 


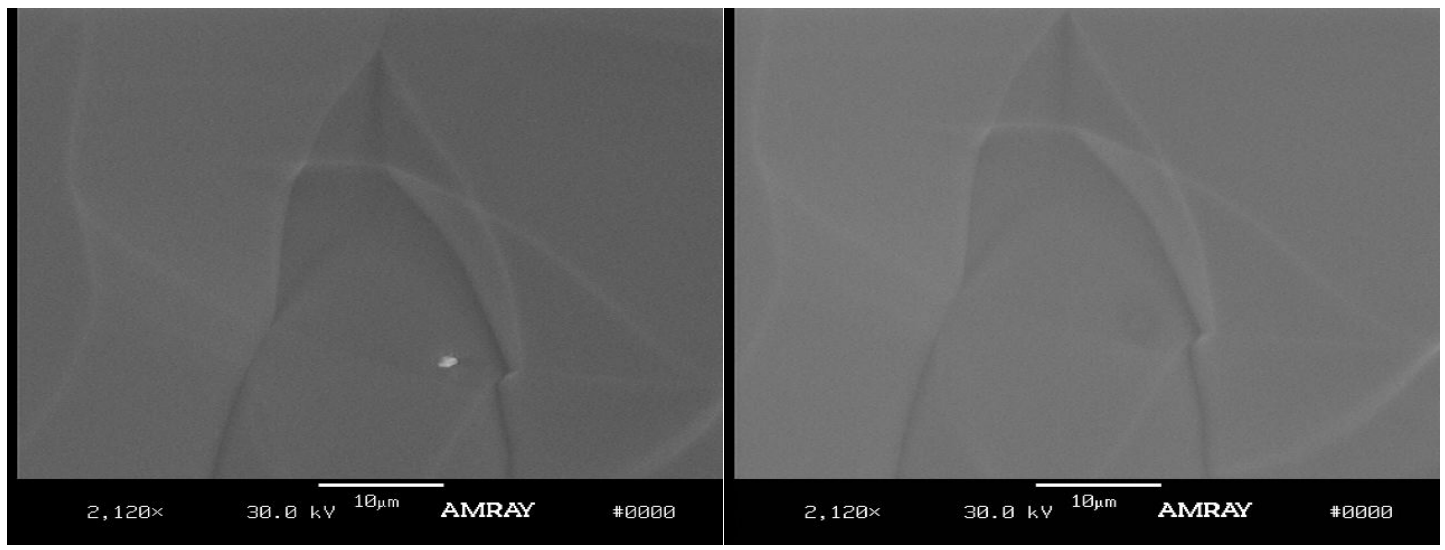

Figure 4.11: The methodology to distinguish external particles from material impurities. A foreign microparticle is shown at the left $(E=136 \mathrm{MV} / \mathrm{m}$, containing $\mathrm{Nb}, \mathrm{Fe}, \mathrm{Cr}, \mathrm{Ni}$ ). After ultrasonic water rinse it disappeared (right).

\subsection{Experimental procedures}

\subsubsection{Experimental circuit}

The circuit block diagram for the experiment control is illustrated in Figure 4.12. The high voltage power supply is controlled by a computer (Pentium $450 \mathrm{MHz}$ running Win98) with a DAQ card from National Instrument (model: PCI-1200). It outputs a voltage ramp from 0 up to $40 \mathrm{kV}$ (depending on the electric field chosen for the field emission scan) in steps of $+200 \mathrm{~V}$, or until a field emission current threshold, usually set at 1-2 nA above the displacement current, is reached, detected by a picoammeter from Keithley (model: 617). The displacement current is constant and is due to the gap capacitance between anode tip and sample, and the voltage ramp. Once the threshold current is reached, the computer will abort the voltage ramp and the maximum voltage that is reached at the anode is recorded to depict the emitting field of the emitter. Hence, detection of strong and weak emitters is accomplished in a single scan. 


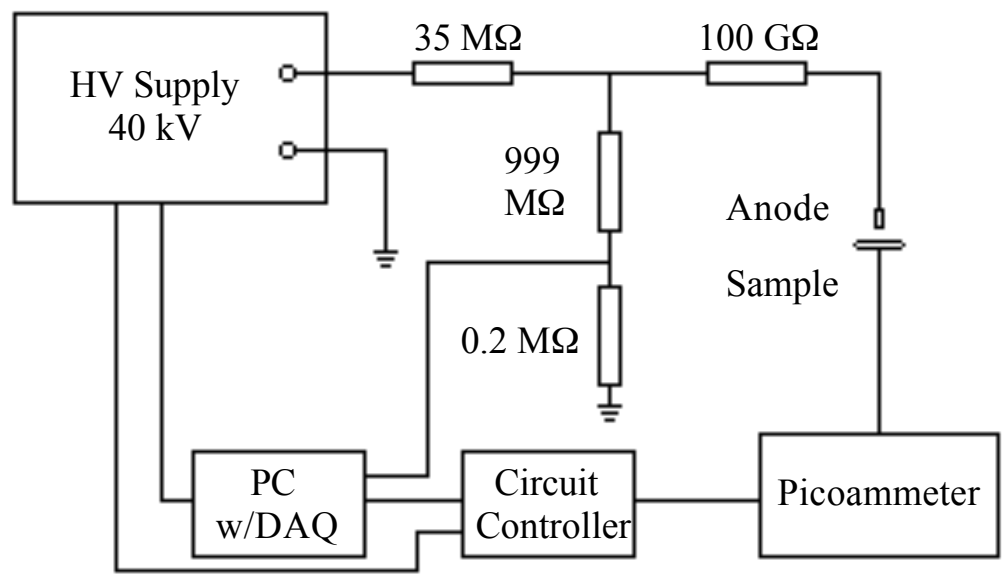

Figure 4.12: Experimental circuit.

The circuit controller, comprising a voltage comparator and other components, is designed to output a signal to immediately disable the high voltage power supply when current exceeds the threshold by a preset amount (usually 2-4 nA above displacement current) to reduce the damage and occurrence of sudden vacuum arcs, which will be discussed in the following chapters. The voltage ramp is generated at a slope of $+200 \mathrm{~V} / 28.6 \mathrm{~ms}$. The time response of the voltage comparator is $45 \mathrm{~ns}$. The controller also acts as a PC data acquisition and analog output interface.

The high resistance (100 G $\Omega$ ) high voltage resistor is custom-made to be vacuum compatible and is placed inside the vacuum chamber in order to reduce the ability of the energy stored in the cable capacitance to damage or destroy emitters during a vacuum arc. The probing voltage at the $0.2 \mathrm{M} \Omega$ resistor is pre-calibrated to the output of the high voltage power supply in the absence of field emission. Its value is measured continuously during each field emission scan by the computer, as is the current flowing through the picoammeter. Both are used to calculate the actual voltage at the anode tip. 


\subsubsection{Entire surface scan}

The gap between anode tip and sample (depends on the electric field chosen for field emission scan, usually set at 100-200 $\mu \mathrm{m}$ ) during each scan is maintained by adjusting the sample position in $z$ while moving in $x, y$ according to the mean surface plane. The mean surface plane is obtained by fitting multi-point profile data for each sample surface. For a planar sample, usually five to nine points dispersed over the surface area are sufficient to produce a good approximation. The multi-point profile data are obtained by moving the sample up in $z$ until it slightly touches the anode tip at the selected points, indicated by a short circuit between sample and anode tip. The resulting coordinates of these selected points $\left(x_{\mathrm{i}}, y_{\mathrm{i}}, z_{\mathrm{i}}\right)$ provide the profile data, from which for every point on the sample surface $(x, y)$, its $z$ coordinate can be obtained. The fitting and calculation are done before each field emission scan using a program written in Matlab. This method results in a gap consistency of about $\pm 10 \mu \mathrm{m}$ determined from the long distance optical microscope. An option in this profiling step is to raise the sample until it is in focus as viewed from the top by the optical microscope while maintaining the parameters of the microscope. The gap consistency is compromised due to the depth of field of the microscope $(\sim 20 \mu \mathrm{m}$ at the current working distance), but this method leaves an untouched surface. An illustrative fitting of the multi-point profile is shown in Figure 4.13.

The FE scan on an entire sample surface can be done using an anode with 150 or $10 \mu \mathrm{m}$ tip radius for coarse to medium resolution scans. The electric field is dependent on anode shape, tip radius and gap distance. Calculations done at University of Geneva show that for a hyperboloid-shaped anode, the electric field correction factor $\kappa$, is given by ${ }^{12}$

$$
\kappa=\frac{V}{E \cdot d}=\frac{1}{2} \sqrt{\frac{r}{d}+1} \cdot\left\{2 \cdot \ln \left(\sqrt{\frac{r}{d}+1}+1\right)-\ln \frac{r}{d}\right\}
$$




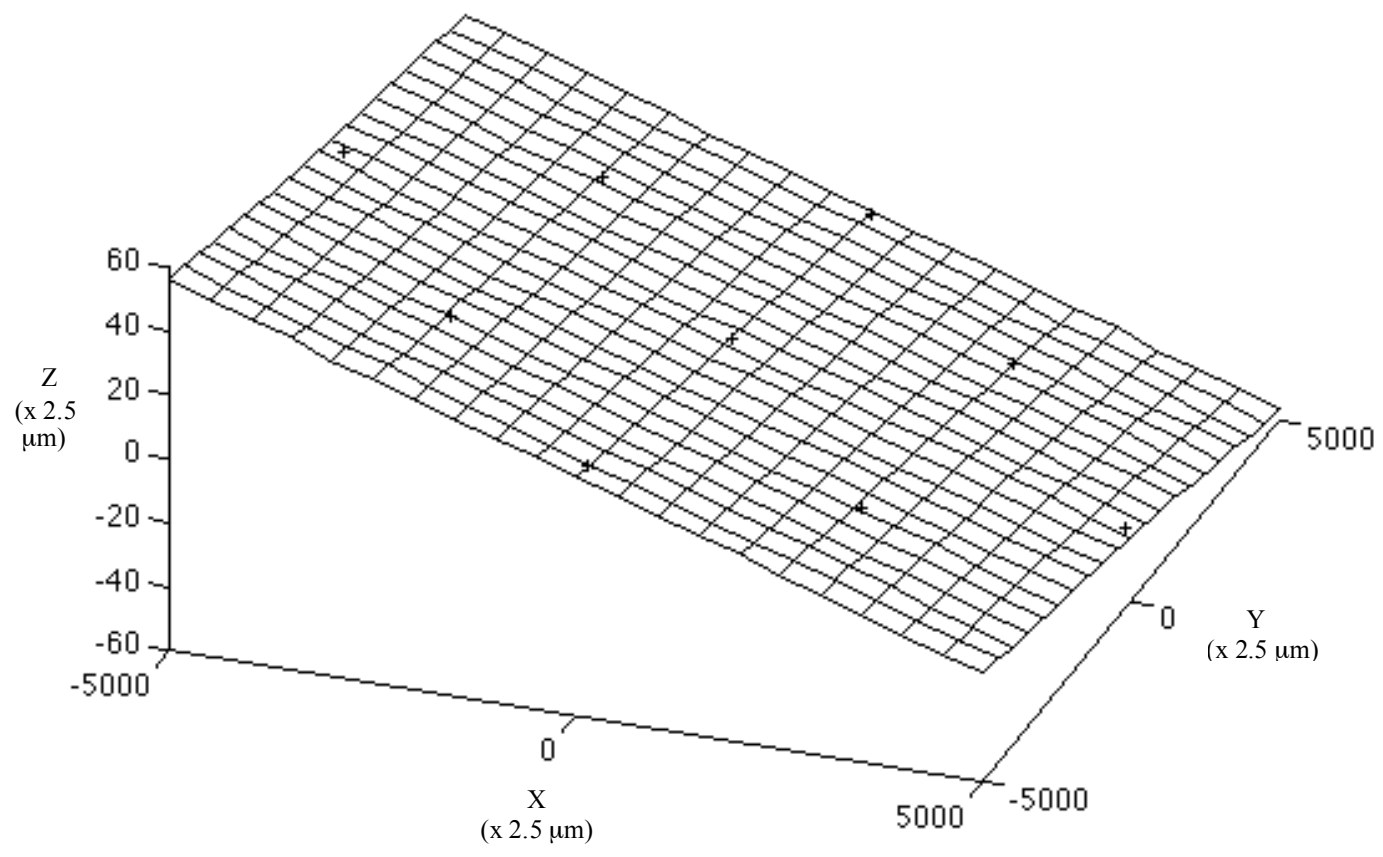

Figure 4.13: Illustrative interpolation and extrapolation of a nine-point profile data set to $25 \mathrm{~mm} \times 25 \mathrm{~mm}$ area. (“+”: profile points)

with $V$ as the applied anode voltage, $d$ as the gap distance from anode apex to cathode plane, $E$ as the electric field at the cathode directly below the anode tip, and $r$ as the curvature of radius of anode tip. For paraboloid-plane geometry, the correction factor can only be calculated by numerical methods. A calculation done by C. S. Athwal at CERN for a paraboloid anode is shown in Figure 4.14 along with results for a hyperboloid (eq. (4.1)). One notes that the results are rather close for the two geometries. In practice when $r \leq d$ (e.g., for our $10 \mu \mathrm{m}$ radius tip), as $d$ changes, the field on the plane will not see the fine detail of the anode apex shape, therefore $r$ can be approximated to change proportionally with $d$, i.e., $r / d \approx c$ where $c$ is a constant. Hence for a microtip anode, $\kappa$ is expected to remain roughly constant for $d$ within a certain range, i.e., $V(d)$ plot is linear at a constant field emission 
current, as confirmed by experimental data, which will be discussed in the next section.

For some tests reported in the following chapters, the entire surface scan was done at a gap of $100 \mu \mathrm{m}$ using the $10 \mu \mathrm{m}$ anode, which is approximately paraboloidshaped as determined from examination under the SEM. The correction factor can be obtained accordingly as above and verified using the $150 \mu \mathrm{m}$ radius cylindrical anode at a small gap (a parallel plane geometry, $E=V / d$ ) at the same emitting site extracting the same amount of field emission current. For other tests discussed in the following chapters, the entire surface scan was done using the $150 \mu \mathrm{m}$ radius cylindrical anode at a gap of $200 \mu \mathrm{m}$, and its correction factor was obtained at a small gap, which will be discussed in the next section.

The lateral distribution of electric field on the sample in the paraboloid-plane setup closely follows the relation: ${ }^{12}$

$$
E(\rho)=\frac{E}{\sqrt{1.3 \cdot\left(\frac{\rho}{d}\right)^{2}+1}},
$$

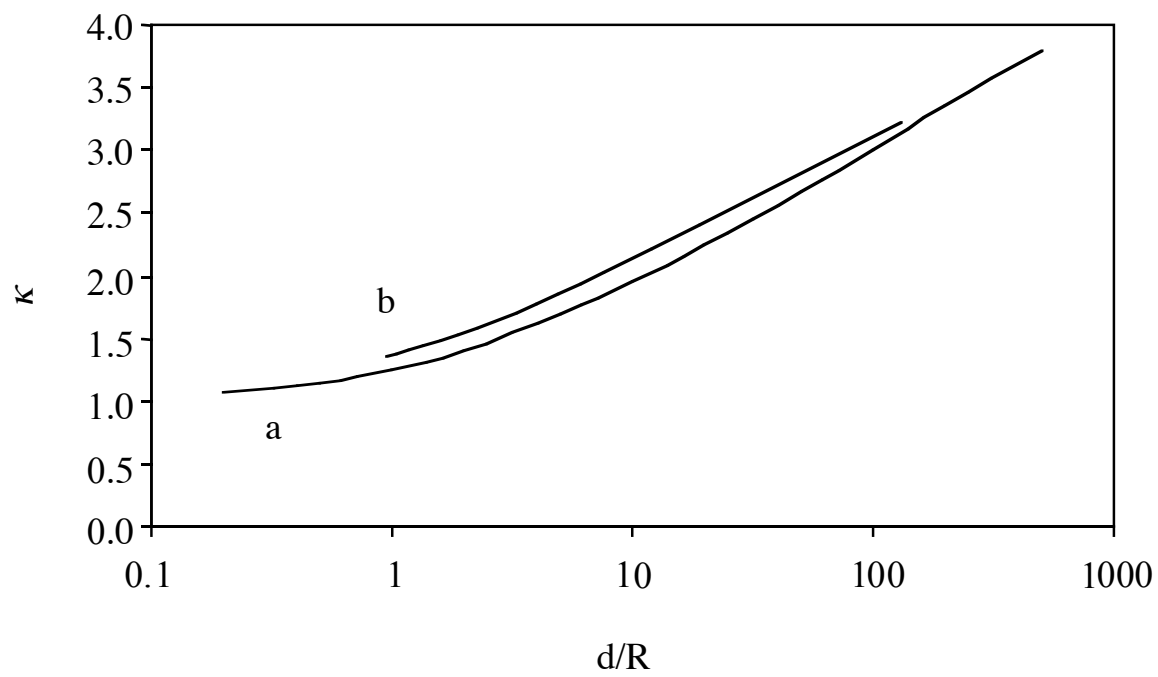

Figure 4.14: Correction factor $\kappa(=V /(E \cdot d))$ versus $d / r$ for hyperboloid-plane (a) and paraboloid-plane geometry (b). ${ }^{12}$ 
where $\rho$ is the lateral distance from anode tip, and $d$ and $E$ are defined as above. $E(\rho) / E$ versus $\rho / d$ is plotted in Figure 4.15.

Measurement of field distribution can be done in the experiment by laterally moving a single emitter to pass directly below the anode tip at a constant voltage after its gap calibration and $\beta, S$ characterization, as discussed in the next section. From $I(\rho)$ and F-N theory, $E(\rho)$ can be obtained. The field distribution measured from a $10 \mu \mathrm{m}$ anode tip at $100 \mu \mathrm{m}$ gap is plotted in Figure 4.16. The lateral distribution for the $150 \mu \mathrm{m}$ anode is similar. As one would notice, the scanning resolution is largely determined by the gap when the tip radius is significantly smaller than gap.

In order to achieve reasonable field flatness, the scan step size needs to be chosen no greater than $1.25 d$ in $x$ and $y$ for

$$
E_{\text {min }}=E\left(\rho=1.25 \cdot d \cdot \frac{\sqrt{2}}{2}\right) \approx 0.70 \cdot E,
$$

according to Eq. 4.2 or the measurement in Figure 4.16. When the step size is chosen as $1.25 d, \sim 80 \%$ of the scanned area sees an electric field between $E$ and $0.8 E$, and $\sim 20 \%$ between $0.8 E$ and $0.7 E$, calculated from the 2-D field distribution. For simplicity purpose, $0.8 E$ is named the "scanning field" in this thesis.

\subsubsection{Local scan and characterization of emitting sites}

After emitters are located, a local fine scan will follow around the emission sites using the $10 \mu \mathrm{m}$ or $1 \mu \mathrm{m}$ radius tip at a gap of $\sim 50 \mu \mathrm{m}$ to accurately locate the emission center. See Figure 4.17 for an illustrative plot of the entire surface scan data, namely $y+C\left(V_{0}-V(x, y)\right)$ versus $x$. $V_{0}$ is the maximum output voltage of the power supply, $V(x, y)$ is the highest voltage reached at $(x, y)$ before the threshold current is exceeded, and $C$ is an adjustment factor to accommodate data display. The local scan at one of the emitting sites is plotted at the right. 


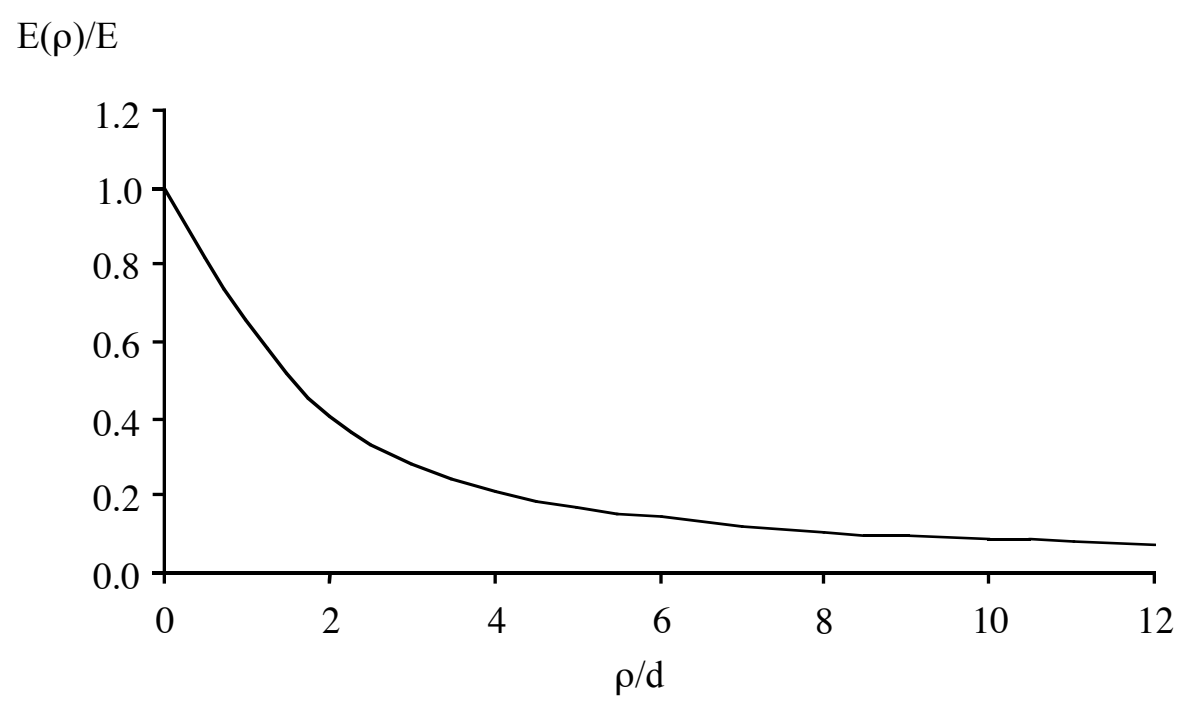

Figure 4.15: The lateral distribution of electric field on sample for paraboloid-plane geometry.

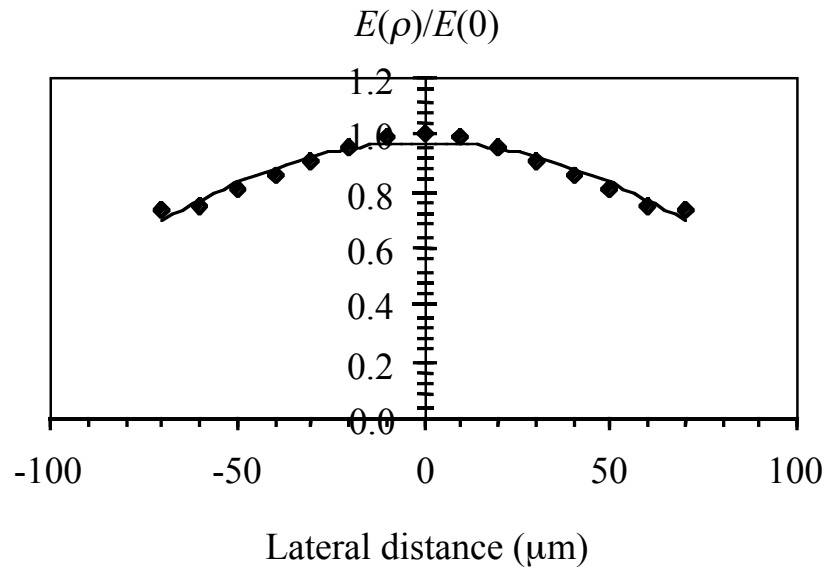

Figure 4.16: Electric field lateral distribution measured for the $10 \mu \mathrm{m}$ radius anode at $100 \mu \mathrm{m}$ gap. 

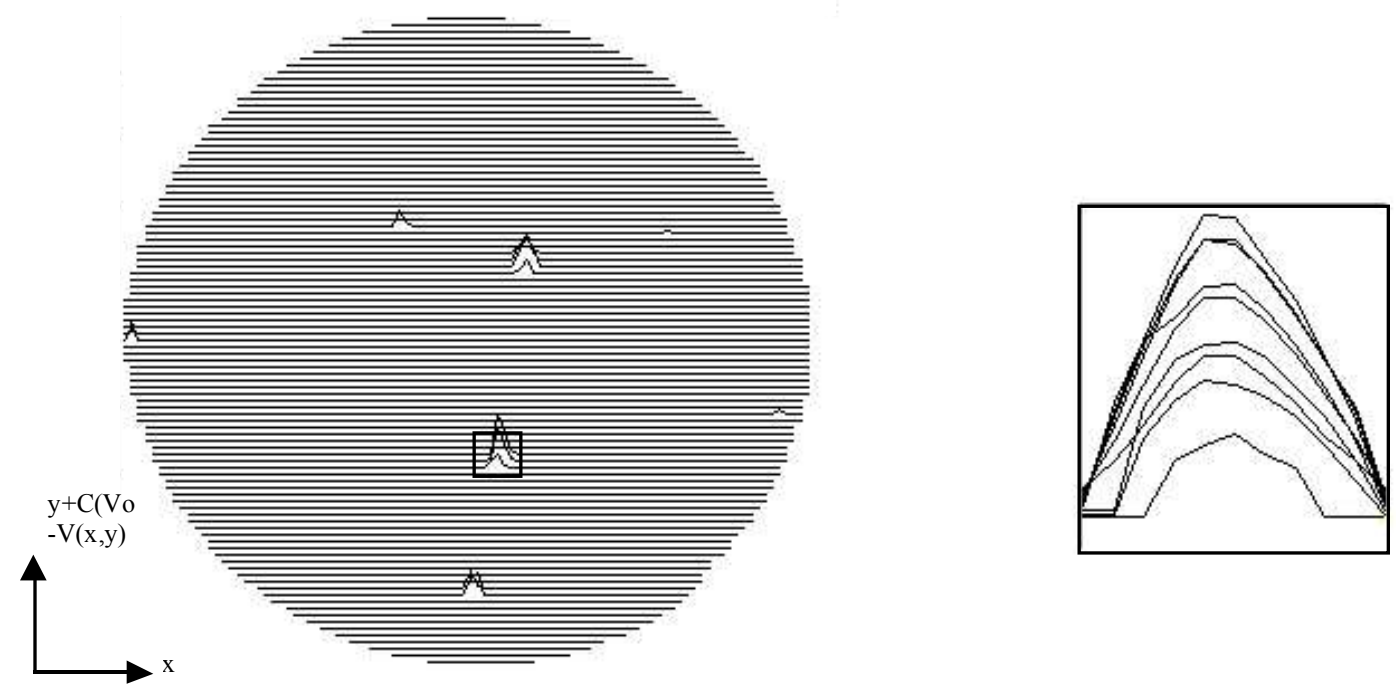

Figure 4.17: Illustration of coarse scan of an entire sample surface $(\phi 25 \mathrm{~mm})$ at 140 $\mathrm{MV} / \mathrm{m}$ using a cylindrical anode of $150 \mu \mathrm{m}$ radius (left), along with local scan of 100 $\mu \mathrm{m} \times 100 \mu \mathrm{m}$ area at the center of the outlined emitter using an anode of $1 \mu \mathrm{m}$ tip curvature radius (right).

The gap can be calibrated at the emitter by centering the anode at the emission center and gradually reducing the gap, e.g., from $50 \mu \mathrm{m}$ to $10 \mu \mathrm{m}$, while adjusting the high voltage to maintain a constant current. $V(d)$ follows a linear trend, explained previously, and the extrapolation of $V(d)$ plot to $V=0$ is set as gap $=0$. The slope of the fitted straight line divided by the correction factor $\kappa$ gives the calibrated electric field, as shown in Figure 4.18. As the gap approaches $100 \mu \mathrm{m}$, $V(d)$ diverts to slightly higher values than the fitted line predicts, and as a result, $\kappa$ is also slightly higher. 


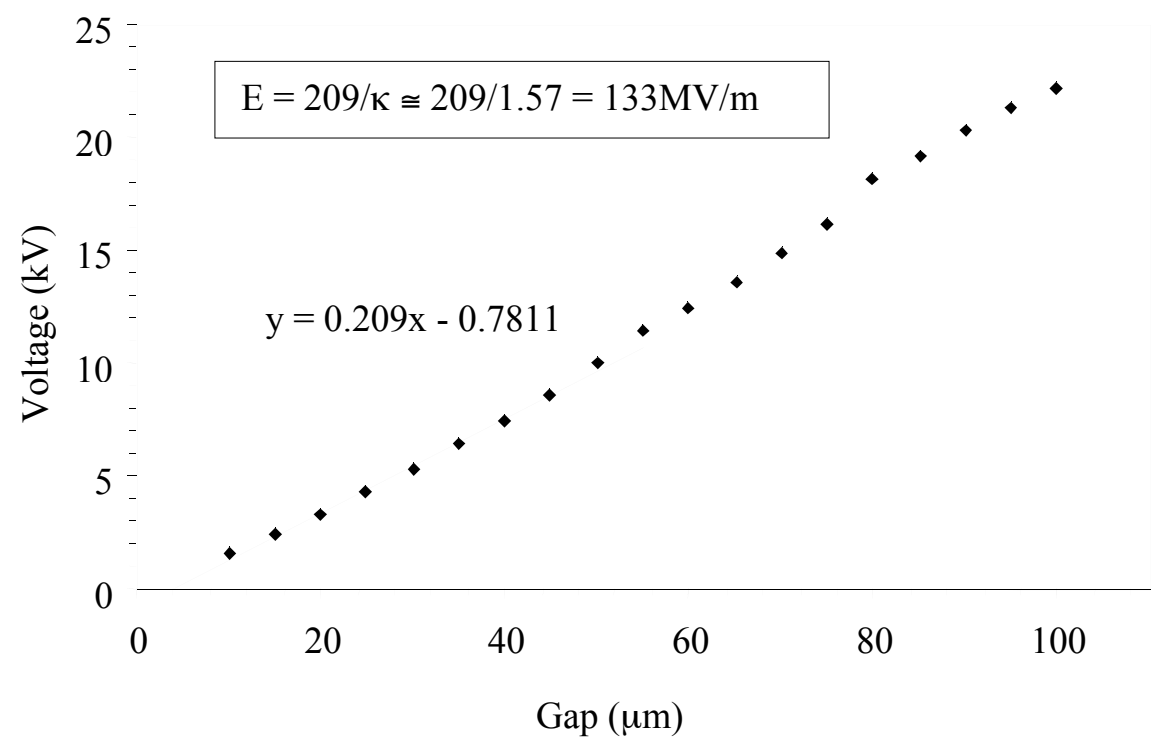

Figure 4.18: Calibration of gap and electric field at the emitting site. Data shown is obtained using a paraboloid-shaped anode of $10 \mu \mathrm{m}$ radius.

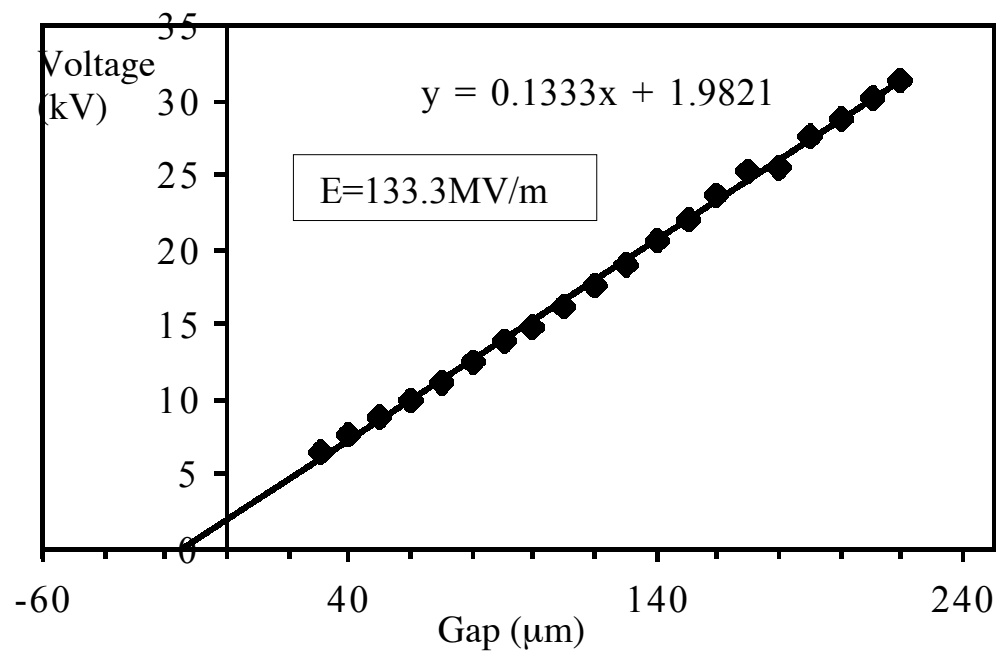

Figure 4.19: The calibration of field correction factor $\kappa$ using the cylindrical anode of $150 \mu \mathrm{m}$ radius at the same emitting site and adjusting the voltage to obtain the same current as in Figure 4.18. 
The field correction factor $\kappa$ can be calibrated using the cylindrical anode with 150 $\mu \mathrm{m}$ radius at the same emitting site, adjusting the voltage to obtain the same current. The slope of $V(d)$ at a small gap is $E$ due to the parallel plane geometry. In fact, the $V(d)$ measurement shows linearity throughout its gap range up to $220 \mu \mathrm{m}$ (Refer to Figure 4.19), thus the slope of $V(d) \cong E$. $E$, if divided by the slope in Figure 4.18 gives the $1 / \kappa$ for the $10 \mu \mathrm{m}$ anode within its gap range.

To characterize an individual emitter, the field enhancement factor $\beta$ and effective emitting area $S$ can be obtained by linear fitting $\ln \left(I / E^{2}\right)$ versus $1 / E$. (Refer to Equation 2.8.) An illustration of F-N plot is shown in Figure 4.20.

\subsubsection{Computerized operation and graphical user interface}

Except for sample loading, unloading and profiling, the experimental operations are executed fully automatically using a computer. The coarse and local scan process, consisting of stepper motor control, instrument control, data acquisition and analys-

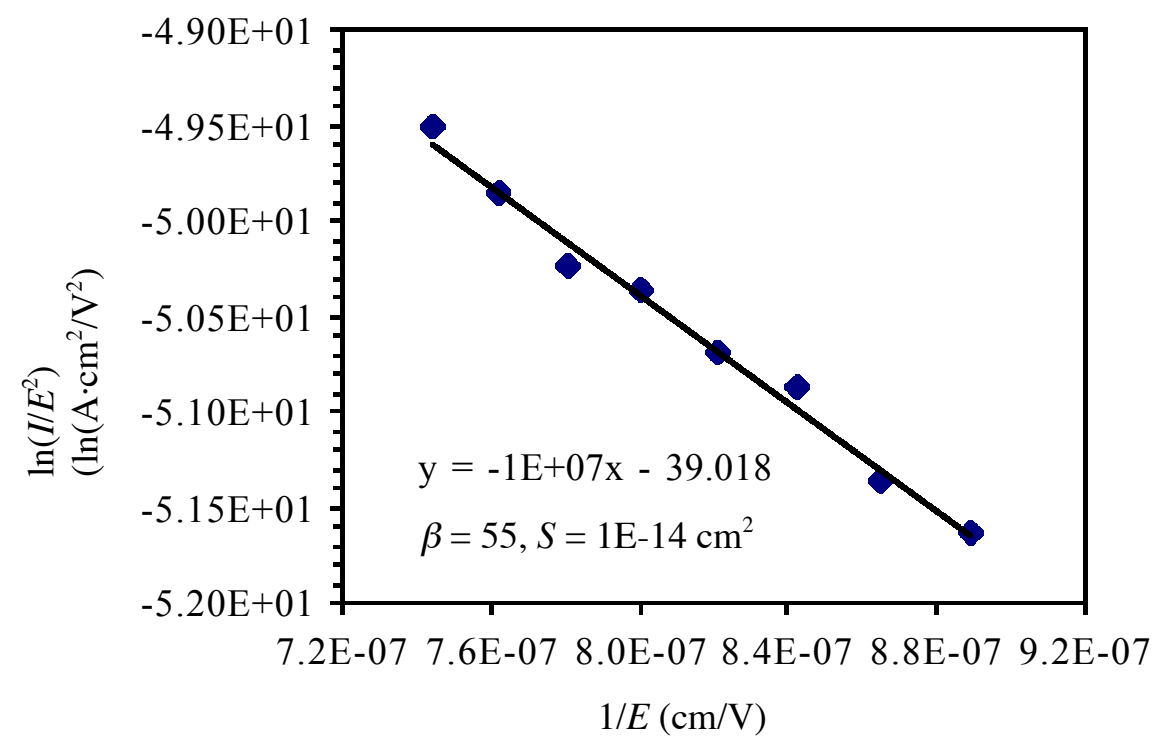

Figure 4.20: $\beta, S$ fitting for the characterization of emitter. 
-is, and image acquisition, are all performed by programs written in LabView. The graphical user interface (the control panel) is illustrated in Appendix A along with an exemplary page of the block diagram.

\subsubsection{In situ examination and material characterization of emitters}

After the emitters are located and characterized, each sample is transferred to the SEM (with EDS) for microscopic study and material characterization of the emitters. The relative probing depth of SEM (secondary electron, $\sim 10 \mathrm{~nm}$ ) and EDX (chracteristic X-ray, $\sim \mu \mathrm{m}$ ) is schematically shown in Figure 4.21.

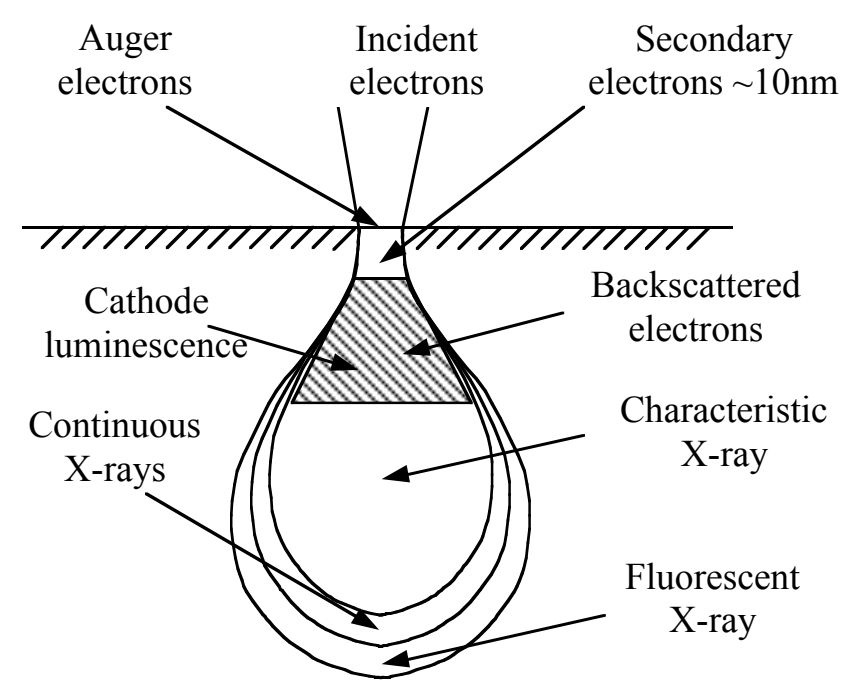

Figure 4.21: Schematic illustration of the typical probing depth of SEM and EDX in a low-density low-atomic-number target. 


\section{Chapter 5}

\section{Enhanced field emission from chemically etched niobium}

\subsection{Chemical etching process and chemistry}

The present EFE study is made on bulk niobium (Nb) surfaces such as are used in SRF resonating cavities employed in particle accelerators. In the same manner as is done with $\mathrm{Nb}$ cavities, a number of $\mathrm{Nb}$ samples made from high purity (RRR 300, $\mathrm{RRR}$ is the ratio of resistance at room temperature to that at low temperature (normal state)) Nb sheet were chemically etched by BCP (buffered chemical polish, $\left.\mathrm{HF}(49 \%): \mathrm{HNO}_{3}(69 \%): \mathrm{H}_{3} \mathrm{PO}_{4}(85 \%)=1: 1: 1\right)$ to remove the machining damaged layer. The chemistry involved consists of two steps as follows:

$$
\begin{gathered}
6 \mathrm{Nb}+10 \mathrm{HNO}_{3} \rightarrow 3 \mathrm{Nb}_{2} \mathrm{O}_{5}+10 \mathrm{NO} \uparrow+5 \mathrm{H}_{2} \mathrm{O} \\
\mathrm{Nb}_{2} \mathrm{O}_{5}+10 \mathrm{HF} \rightarrow 2 \mathrm{NbF}_{5}+5 \mathrm{H}_{2} \mathrm{O} \\
\mathrm{Nb}_{2} \mathrm{O}_{5}+10 \mathrm{HF} \rightarrow 2 \mathrm{H}_{2} \mathrm{NbOF}_{5}+3 \mathrm{H}_{2} \mathrm{O} .
\end{gathered}
$$

Nitric acid is an oxidizing agent towards $\mathrm{Nb}$, producing niobium pentoxide $\mathrm{Nb}_{2} \mathrm{O}_{5}$, as shown in Eq. 5.1. Hydrofluoric acid and phosphoric acid are not oxidizing agents for $\mathrm{Nb}$. Niobium pentoxide will in turn react with $\mathrm{HF}$ and produce soluble niobium pentafluoride $\mathrm{NbF}_{5}$ (Eq. 5.2), or its hydrated form $\mathrm{H}_{2} \mathrm{NbOF}_{5}$ (Eq. 5.2'). The sum effect of nitric acid and hydrofluoric acid is:

$$
6 \mathrm{Nb}+10 \mathrm{HNO}_{3}+30 \mathrm{HF} \rightarrow 6 \mathrm{NbF}_{5}+10 \mathrm{NO} \uparrow+20 \mathrm{H}_{2} \mathrm{O} .
$$


Phosphoric acid acts as a buffer in the acid mixture. BCP is usually accompanied by a vigorous brownish gas evolution (Eq. 5.1). To explain the polishing mechanism of $\mathrm{BCP}$, some have suggested that a viscous layer of reaction products only forms in recesses of the $\mathrm{Nb}$ surface and prevents the direct access of acid to these areas, while the viscous layer at surface protrusions could be swept away by the turbulent flow of the acid mixture due to the gas generation. ${ }^{61}$ As a result, the $\mathrm{Nb}$ surface is smoothed.

To remove acid residue and particles on samples introduced during handling, ultrasonic cleaning in de-ionized water was performed immediately following BCP. Ultrasonic rinse usually uses frequencies between 20 and $80 \mathrm{kHz}$. Cavitation, implosion of bubbles, and scrubbing action are involved in the cleaning process. ${ }^{62}$ Because cavitation can occur at recesses, these areas can be cleaned as well. Some samples in this chapter were blown dry by filtered nitrogen gas, while others were methanol rinsed following the ultrasonic rinse to displace water from the surface, and then placed on a filtered laminar flow bench in the cleanroom for a few minutes until dry.

\subsection{Summary of preliminary test results}

About $20 \mathrm{Nb}$ samples were BCP (1:1:1) etched for various amounts of removal and scanned for field emitters at $\sim 70$ or $140 \mathrm{MV} / \mathrm{m}$. Generally, five types of emitters were located: geometrical damage (scratches), particles containing foreign elements, features with no foreign elements detectable by EDS, geometrical irregularities that may have been caused by the non-uniformity of etching, and emitters with no distinctive or resolvable features. There were also sites that were completely destroyed by vacuum arcs. Although they may not all be field emission initiated, they will be listed as a category of emitters in this thesis-emitters destroyed by vacuum arc-for simplicity. 
Geometrical damage consists of un-removed machining damage and new scratches caused by handling after BCP. Some machining damage sites contain foreign elements detected by EDS. In order to reduce this category of emitters, changes in sample handling were made, including (1) use a polyethylene fixture to secure samples in their places to avoid accidental collision during and after BCP; (2) put the fixture along with samples in a polyethylene basket to allow immediate removal from acid after BCP for water rinse and avoid touching the sample surfaces by any tools; (3) use tweezers to handle sample by the grooves at the back side to prevent scratches on the surface; and (4) make sure that the surface is never touched by any means after BCP. After the above changes, geometrical damage was reduced to zero after a sufficient amount of removal by BCP, but the other five categories of emitters remain. Furthermore, even with a similar and significant amount of removal, emitter density still varies significantly from one sample to another, e.g., a total of 8 emitters for the best sample, and over 60 emitters for the worst.

\subsubsection{Categories of observed emitters}

Some typical geometrical damage sites identified as field emitters are shown in Figure 5.1. The majority of emitters, however, are foreign particles or contaminants, with various elemental compositions. At some emitting sites, features were found but no foreign elements were detected by EDS, possibly due to the quite large probing depth of EDS relative to the probing depth of SEM. A very few emitters were found to be geometrical irregularities, possibly caused by the inhomogeneity in etching, with no visible foreign particle or detectable contaminant. Refer to Figure 5.2, 5.3 and 5.4 for pictures of the last three categories of emitters. Their emitting field (for $2 \mathrm{nA}$ field emission current detection, as used throughout this thesis), elemental composition detected by EDS, and in some cases the F-N fitting parameters, i.e., $\beta$ and $S$, are listed for the emitters as well. Finally, for a small number of emitting sites, no distinctive features were found. 


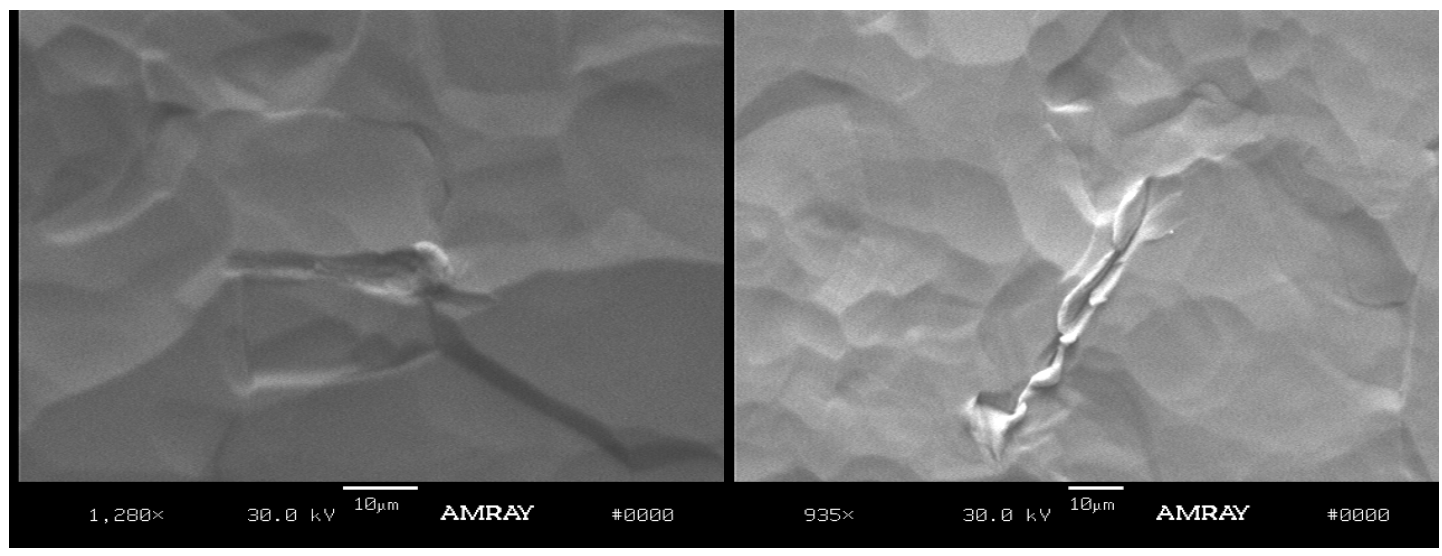

$$
\begin{array}{cl}
\mathrm{Nb}, \beta & =100, S=3 \mathrm{E}-13 \mathrm{~cm}^{2}, \quad \mathrm{Nb}, E=36 \mathrm{MV} / \mathrm{m} . \\
E & =57 \mathrm{MV} / \mathrm{m} .
\end{array}
$$

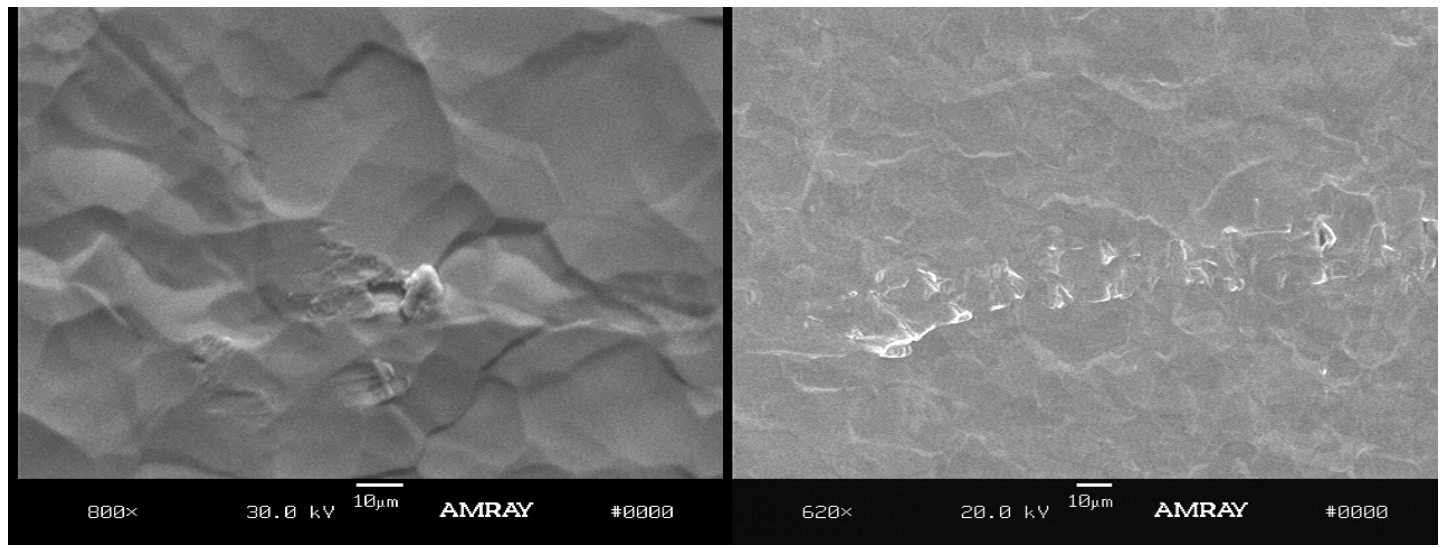
$\mathrm{Nb}, E=62 \mathrm{MV} / \mathrm{m}$.
$\mathrm{Nb}, E=30 \mathrm{MV} / \mathrm{m}$.

Figure 5.1: Geometrical damages as field emitters. The emitting field (for $2 \mathrm{nA}$ detected field emission current), elemental composition detected by EDS, and in some cases the F-N fitting parameters $\beta$ and $S$ of emitters are listed. 


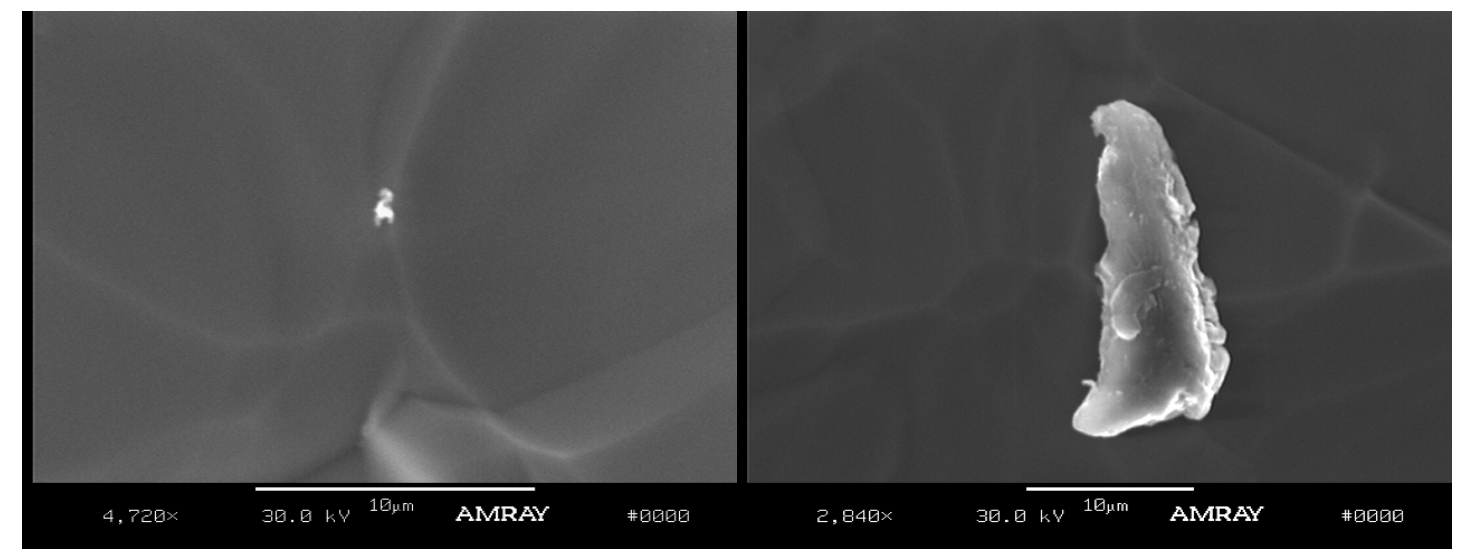
$\mathrm{Nb}, \mathrm{Fe}, \mathrm{Cr}, \mathrm{Ni} ., E=95 \mathrm{MV} / \mathrm{m}$.
$\mathrm{Nb}, \mathrm{Cr}, \mathrm{Fe}, \mathrm{Ni}, E=52 \mathrm{MV} / \mathrm{m}$.

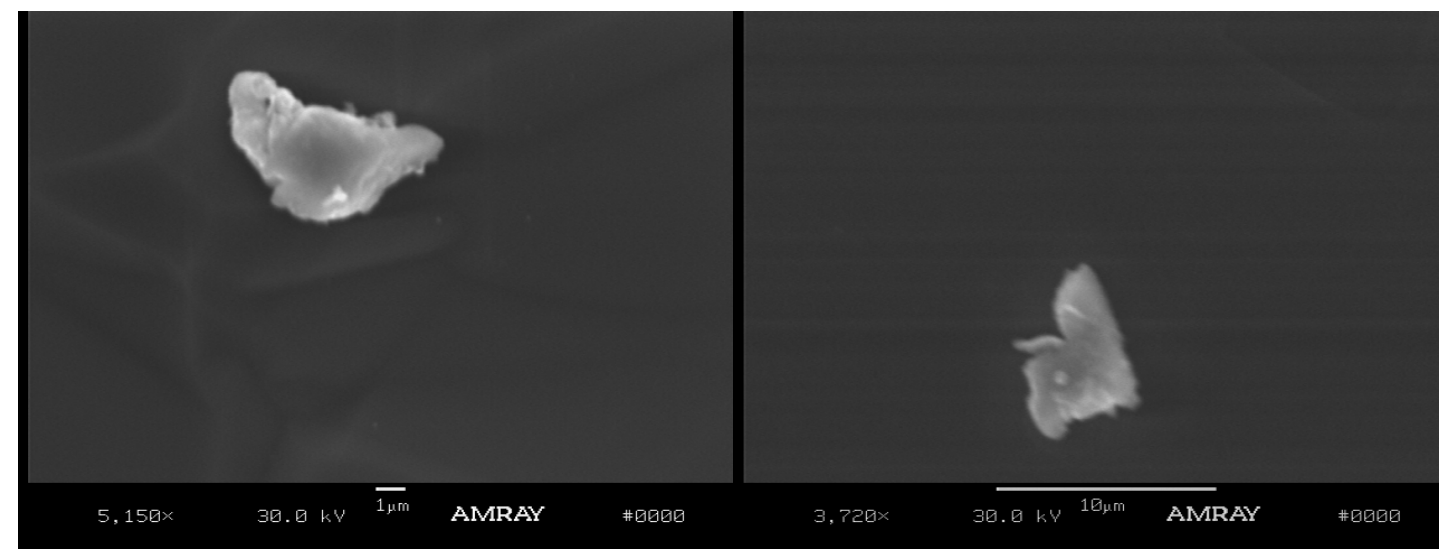

$\mathrm{Nb}, \mathrm{Cr}, \mathrm{Fe}, \mathrm{Ni}, \mathrm{Si}, E=47 \mathrm{MV} / \mathrm{m} . \quad \mathrm{Nb}, \mathrm{Fe}, \mathrm{Cr}, \mathrm{Ni}, E=19 \mathrm{MV} / \mathrm{m}$.

Figure 5.2: Some pictures of foreign particles or contaminants as field emitters. 


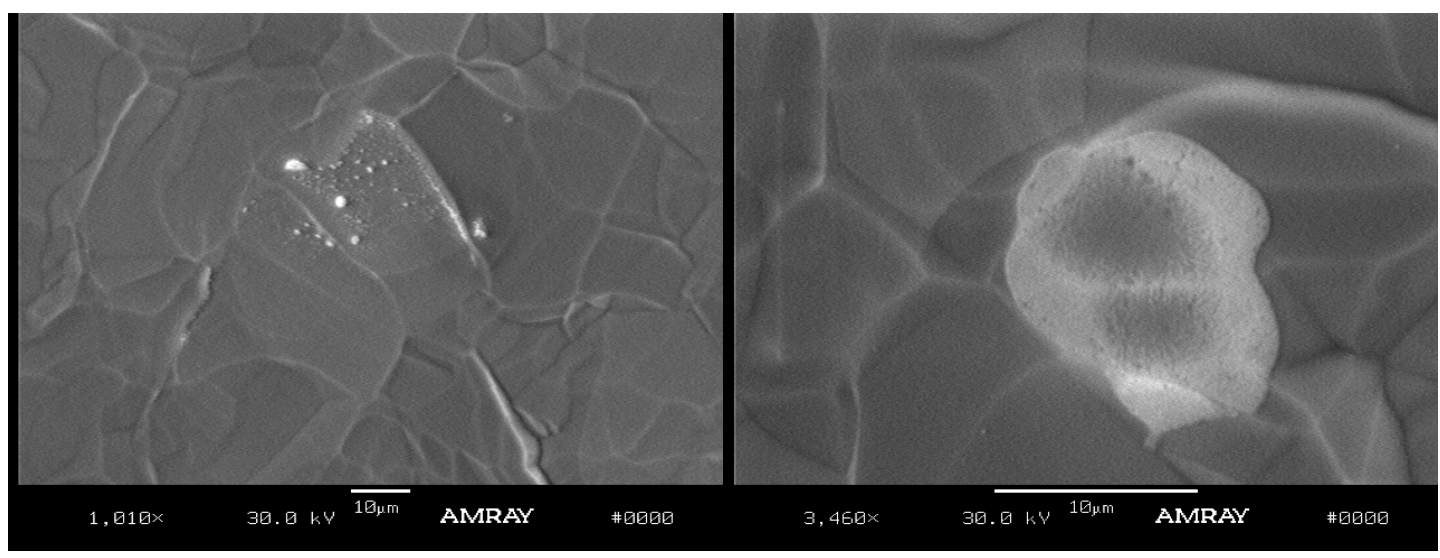

$\mathrm{Nb}, E=69 \mathrm{MV} / \mathrm{m}$.

$\mathrm{Nb}, E=62 \mathrm{MV} / \mathrm{m}$.

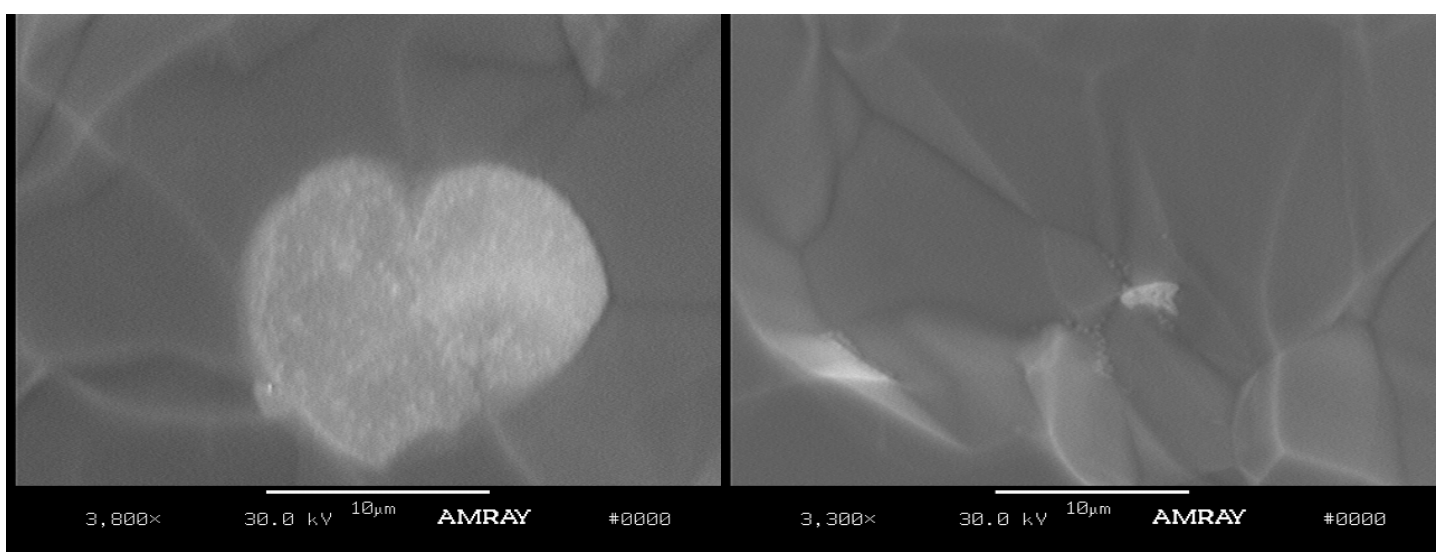

$\mathrm{Nb}, E=38 \mathrm{MV} / \mathrm{m}$.

$\mathrm{Nb}, E=84 \mathrm{MV} / \mathrm{m}$.

Figure 5.3: Emitting features with no foreign elements detected by EDS. 


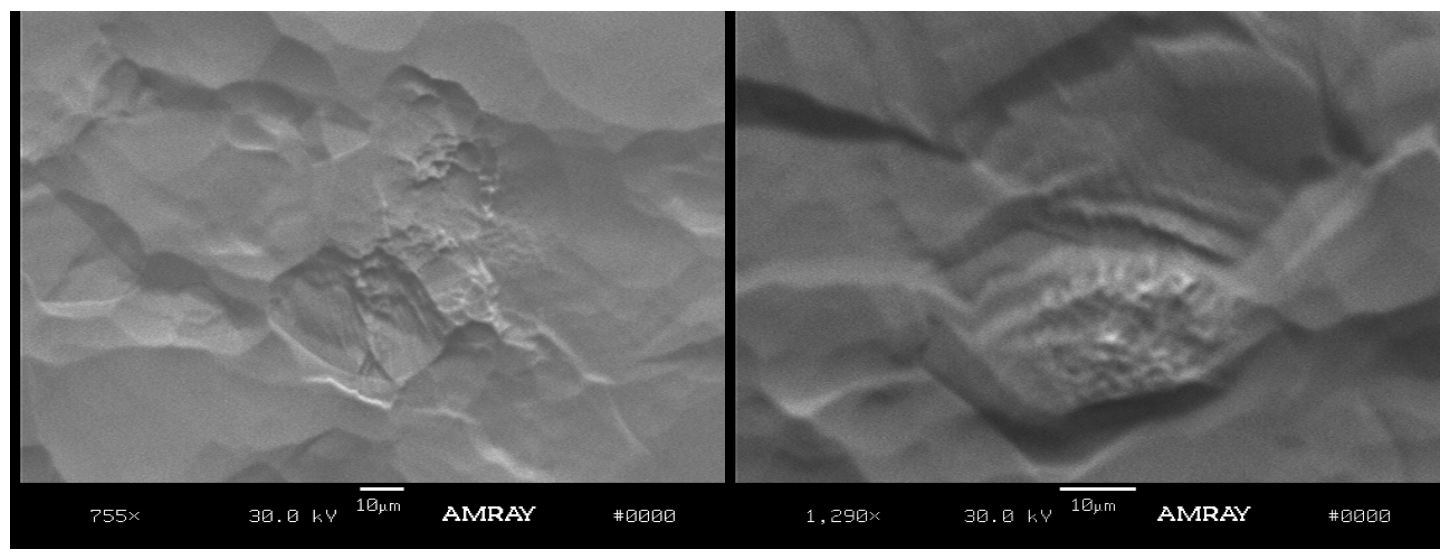
$\mathrm{Nb}, \beta=180, S=1.6 \mathrm{E}-13 \mathrm{~cm}^{2}$,
$\mathrm{Nb}, \beta=200, S=4.7 \mathrm{E}-13 \mathrm{~cm}^{2}$, $E=67 \mathrm{MV} / \mathrm{m}$. $E=67 \mathrm{MV} / \mathrm{m}$.

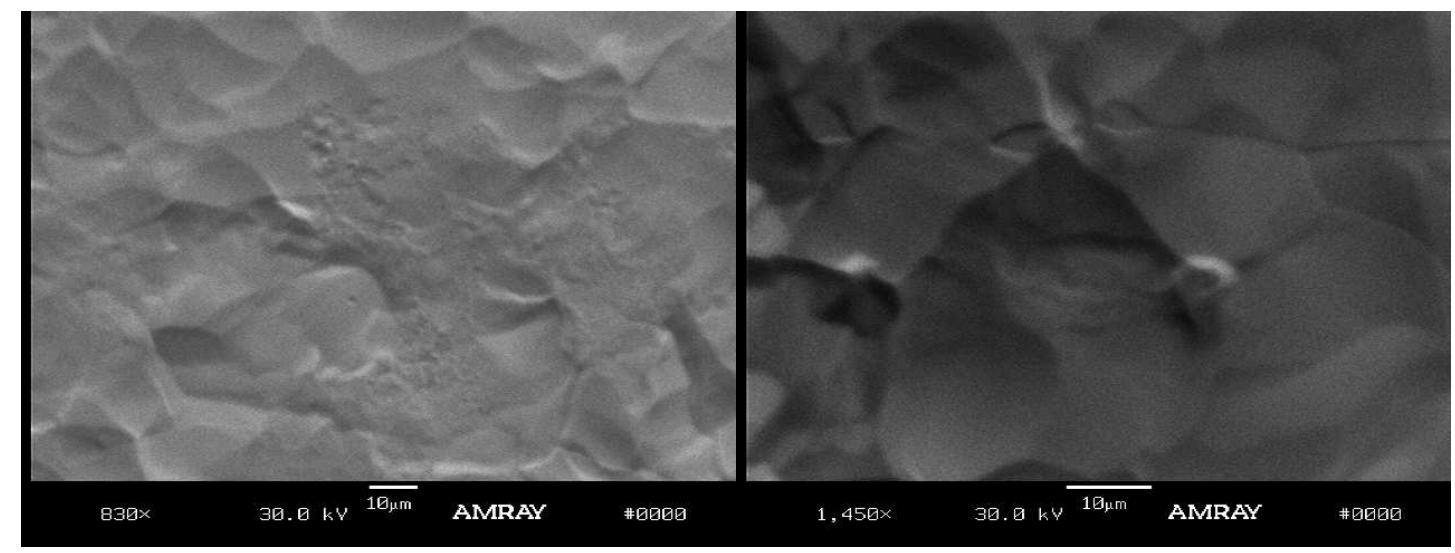

$$
\begin{array}{cl}
\mathrm{Nb}, \beta=110, S=1.5 \mathrm{E}-11 \mathrm{~cm}^{2}, \quad \mathrm{Nb}, E=54 \mathrm{MV} / \mathrm{m} \\
E=67 \mathrm{MV} / \mathrm{m} .
\end{array}
$$

Figure 5.4: Geometrical irregularities as field emitters, no foreign elements detected. 


\subsubsection{Statistics of emitters}

The majority of emitters from the $\sim 20$ samples were located and analyzed. A subset of these emitters were characterized according to the modified F-N parameterization for their geometrical enhancement factors, $\beta$, and effective emitting areas, $S$. A small selection of these emitters are listed in table 5.1. An illustrative EDS spectrum at one emitting site is shown in Figure 5.5.

Table 5.1: A small selection of analyzed emitters from the initial 20 samples after $150 \mu \mathrm{m}$ - $450 \mu \mathrm{m}$ removal by BCP unless otherwise noted. \#9-1(3), e.g., is from sample $\# 9$, after the 1 st surface removal $\left(2^{\text {nd }}, 3^{\text {rd }}, \ldots .\right.$. is after additional removal $)$, on emitting site No. 3.).

\begin{tabular}{|c|c|c|c|c|}
\hline Emitter & $\begin{array}{c}\text { Emitting field } \\
(\mathrm{MV} / \mathrm{m})\end{array}$ & $\begin{array}{c}\text { Elements detected by } \\
\text { EDS }\end{array}$ & $\bar{\beta}$ & $S\left(\mathrm{~cm}^{-2}\right)$ \\
\hline$\# 9-1(3)$ & 56 & $\mathrm{Nb}$ & 202 & $4.7 \mathrm{E}-13$ \\
\hline$\# 9-1(4)$ & 56 & $\mathrm{Nb}$ & 180 & $1.6 \mathrm{E}-13$ \\
\hline$\# 7-1(46)$ & 103 & $\mathrm{Nb}, \mathrm{Fe}, \mathrm{Al}$ & & \\
\hline$\# 8-1(2)$ & 89 & $\begin{array}{c}\mathrm{Al}, \mathrm{Si}, \mathrm{Ca}, \\
\mathrm{Ba}, \mathrm{Nb}\end{array}$ & & \\
\hline$\# 8-1(39)$ & 47 & $\overline{\mathrm{Nb}, \mathrm{Fe}}$ & & \\
\hline$\# 25-1(1)$ & 93 & $\begin{array}{l}\text { Nb,Fe,Cr, } \\
\text { Ni,Mn } \\
\end{array}$ & & \\
\hline$\# 25-1(5)$ & 135 & $\mathrm{Nb}, \mathrm{Ca}$ & & \\
\hline$\# 25-1(8)$ & 117 & $\mathrm{Nb}, \mathrm{C}$ & & \\
\hline$\# 25-1(39)$ & 135 & $\mathrm{Nb}, \mathrm{Fe}$ & & \\
\hline$\# 32-1(1)$ & 93 & $\mathrm{Nb}, \mathrm{Fe}, \mathrm{Cr}, \mathrm{Ni}$ & & \\
\hline$\# 32-1(2)$ & 47 & $\begin{array}{l}\mathrm{Nb}, \mathrm{Cr}, \mathrm{Fe} \\
\mathrm{Ni}, \mathrm{Si} \\
\end{array}$ & & \\
\hline$\# 32-1(10)$ & 117 & $\mathrm{Nb}, \mathrm{Fe}, \mathrm{Cr}, \mathrm{Ni}$ & & \\
\hline$\# 32-1(11)$ & 51 & $\mathrm{Nb}, \mathrm{Cr}, \mathrm{Fe}, \mathrm{Ni}$ & & \\
\hline$\# 32-1(14)$ & 89 & $\begin{array}{l}\mathrm{Nb}, \mathrm{Ca}, \mathrm{Al}, \\
\mathrm{Zn}, \mathrm{Fe}, \mathrm{C}\end{array}$ & & \\
\hline$\# 32-1(17)$ & 70 & $\mathrm{Nb}, \mathrm{Cu}$ & & \\
\hline \#34-1(1)-EP & 34 & $\mathrm{Nb}, \mathrm{Fe}$ & & \\
\hline$\# 34-1(2)-\mathrm{EP}$ & 33 & $\mathrm{Nb}, \mathrm{Fe}, \mathrm{Cr}, \mathrm{Ni}$ & & \\
\hline \#34-1(3)-EP & 19 & $\mathrm{Nb}, \mathrm{Fe}, \mathrm{Cr}, \mathrm{Ni}$ & & \\
\hline$\# 34-2(1)$ & 140 & $\mathrm{Nb}, \mathrm{Fe}$ & & \\
\hline$\# 34-2(15)$ & 95 & $\mathrm{Nb}, \mathrm{Fe}$ & & \\
\hline$\# 54-1(1)$ & 46 & $\mathrm{Nb}$ & 87 & $1.5 \mathrm{E}-12$ \\
\hline$\# 54-1(2)$ & 94 & $\begin{array}{l}\mathrm{Nb}, \mathrm{Ca}, \mathrm{Si}, \\
\mathrm{Al}, \mathrm{C}, \mathrm{Zn}\end{array}$ & 34 & $8.26 \mathrm{E}-11$ \\
\hline
\end{tabular}




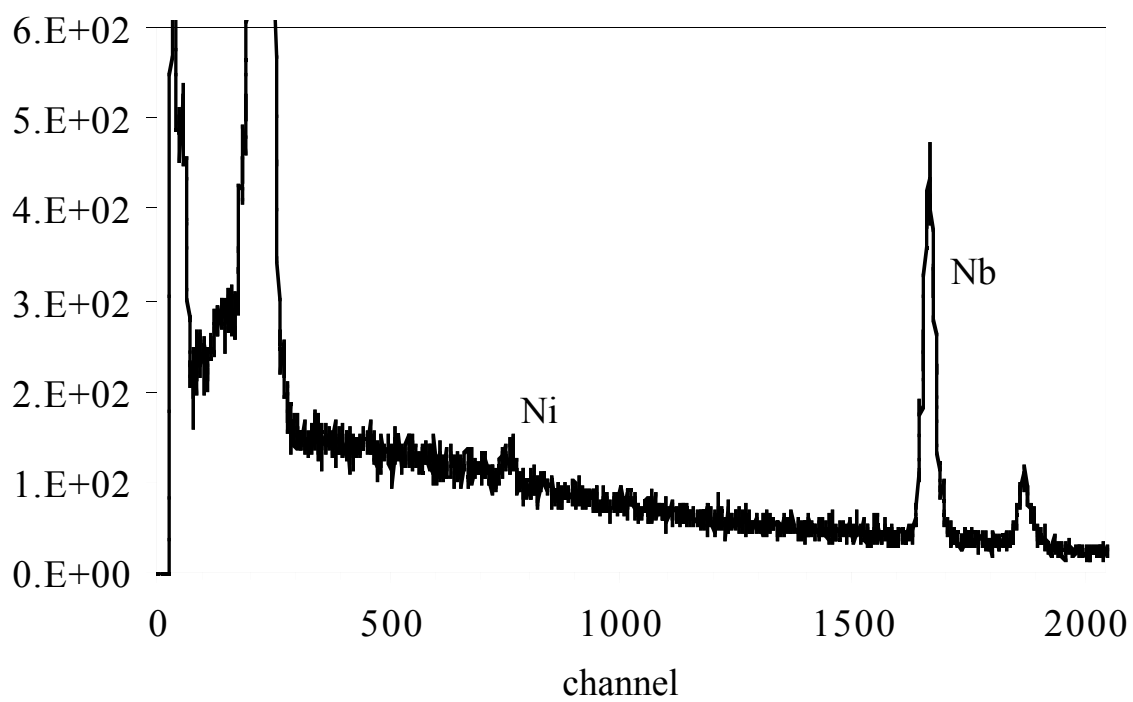

Figure 5.5: An illustrative spectrum of EDS at one emitting site. EP: eletropolishing.

\subsection{Results after improvement in machining process}

\subsubsection{Improvement in machining process}

In order to improve the reproducibility of the field emission performance, the machining process was examined first as $\mathrm{Nb}$ is a soft, abrasive metal whose machining process is not easily controlled. A few rules, as listed below, were proposed and followed for new samples.

- inspect $\mathrm{Nb}$ sheet to choose defect-free material

- use a designated clean area for the machining

- use only plastic fixtures in the machining to minimize the damage to the sample surface

- change machining tools frequently as dull tools will embed impurities into the sample 


\subsubsection{Field emission test results}

New samples were made from a $\mathrm{Nb}$ sheet of $\sim 300 \mathrm{RRR}$ that was closely examined to be defect-free. They were then BCP etched to remove various amount of material from the surfaces, followed by ultrasonic rinse in DI water (UWR). Finally, samples were methanol rinsed and laminar air-flow dried in a cleanroom before being scanned for field emission. The results at $\sim 140 \mathrm{MV} / \mathrm{m}$ are listed in Table 5.2.

Table 5.2: Field emitters from BCP samples after improvement in machining (Field emission scanning field $140 \mathrm{MV} / \mathrm{m}$, indicated fields are the emitting fields that yield $2 \mathrm{nA} \mathrm{FE}$ current, the scan area for each sample is $25 \mathrm{~mm}$ dia.)

\begin{tabular}{|c|c|c|c|}
\hline & Foreign particles & $\begin{array}{c}\text { Features with } \mathrm{Nb} \\
\text { detected only }\end{array}$ & $\begin{array}{c}\text { Emitters destroyed } \\
\text { by vacuum arc }\end{array}$ \\
\hline $\begin{array}{c}\# 65-1 \\
(250 \mu \mathrm{m} \mathrm{BCP})\end{array}$ & \begin{tabular}{l}
\multicolumn{1}{c}{2} \\
{$[40 \mathrm{MV} / \mathrm{m}(\mathrm{Nb}, \mathrm{C}$} \\
$\mathrm{Cu}, \mathrm{Ca}, \mathrm{Fe}, \mathrm{Ni})$ \\
$86 \mathrm{MV} / \mathrm{m}(\mathrm{Nb}, \mathrm{W}$ \\
$\mathrm{Ni}, \mathrm{O})]$
\end{tabular} & 0 & 1 \\
\hline $\begin{array}{c}\# 65-2 \\
(280 \mu \mathrm{m} \text { BCP })\end{array}$ & \begin{tabular}{l}
\multicolumn{1}{c}{2} \\
{$[140 \mathrm{MV} / \mathrm{m}(\mathrm{Nb}$,} \\
$\mathrm{Fe}), 114 \mathrm{MV} / \mathrm{m}$ \\
$(\mathrm{Nb}, \mathrm{Fe}, \mathrm{Cr})]$
\end{tabular} & $\begin{array}{c}3 \\
{[130 \mathrm{MV} / \mathrm{m}, 118} \\
\mathrm{MV} / \mathrm{m}, 135 \mathrm{MV} / \mathrm{m}]\end{array}$ & 2 \\
\hline $\begin{array}{c}\# 65-3 \\
(360 \mu \mathrm{m} \mathrm{BCP})\end{array}$ & $\begin{array}{l}1 \\
{[136 \mathrm{MV} / \mathrm{m}(\mathrm{Nb},} \\
\mathrm{Fe}, \mathrm{Cr}, \mathrm{Ni})]\end{array}$ & 0 & 2 \\
\hline $\begin{array}{c}\# 63-1 \\
(250 \mu \mathrm{m} \mathrm{BCP})\end{array}$ & \begin{tabular}{l}
\multicolumn{1}{c}{3} \\
{$[100 \mathrm{MV} / \mathrm{m}(\mathrm{Nb}$,} \\
$\mathrm{Fe}, \mathrm{Cr}), 42 \mathrm{MV} / \mathrm{m}$ \\
$(\mathrm{Ag}, \mathrm{Nb}), 105$ \\
$\mathrm{MV} / \mathrm{m}(\mathrm{Nb}, \mathrm{Fe}$, \\
$\mathrm{Cr})]$
\end{tabular} & $\begin{array}{c}1 \\
{[133 \mathrm{MV} / \mathrm{m}]}\end{array}$ & 4 \\
\hline $\begin{array}{c}\# 63-2 \\
(330 \mu \mathrm{m} \text { BCP }) \\
\end{array}$ & 0 & $\begin{array}{c}1 \\
{[127 \mathrm{MV} / \mathrm{m}]}\end{array}$ & 4 \\
\hline $\begin{array}{c}\# 66-1 \\
(330 \mu \mathrm{m} \text { BCP })\end{array}$ & $\begin{array}{c}1 \\
{[120 \mathrm{MV} / \mathrm{m}(\mathrm{Nb})]}\end{array}$ & $\overline{0}$ & 1 \\
\hline
\end{tabular}


The previous five categories of emitters now have dropped to three, i.e., geometrical irregularities and emitters with no discernible features were not found from this group of samples, which indicates that these emitters might be related to the previous sample surfaces that were not quite contaminant-free. For instance, the non-uniform chemical etching at contaminated sites could cause the geometrical irregularities or etch pits, while traces of impurities embedded at grain boundaries could cause field emission that is impossible or very difficult to identify.

Seven of the nine foreign particulate emitters contain $\mathrm{Fe}, \mathrm{Cr}$ and $\mathrm{Ni}$ or a subset of these elements, along with other foreign elements and Nb. They could originate from machining tools made of stainless steel. The rest of the emitters contain Ag and $\mathrm{Nb}$, and $\mathrm{Nb}$ respectively. They emit at a wide range of electric fields, from 40 $\mathrm{MV} / \mathrm{m}$ up to $140 \mathrm{MV} / \mathrm{m}$. They are categorized as foreign particles because of their appearances and/or the fact that they are completely or partly washed away by further ultrasonic water rinse, similar to that shown in Figure 4.11. The sample edges, although not within the FE scan area, are found to have many machining damage and contaminants under SEM and EDS. These contaminants could be a source for the identified particulate emitters rich in stainless steel, e.g., if they are rinsed off into the DI water medium by ultrasonic rinse and then re-deposited onto the sample surfaces. As a result, further improvement in machining was pursued to reduce this category of field emitters, which will be described in the following section.

Emitters were also found that appear bright but not particle-like under the SEM and do not have any foreign elements detected by EDS. The several micron profiling depth of EDS makes it unsuitable to detect very superficial elements. Nonetheless, these emitters only emit at $\geq 120 \mathrm{MV} / \mathrm{m}$, which is significantly higher than normally achieved or currently desired peak surface fields in cavities.

The last category of emitters are those completely destroyed by vacuum arcs. These often occurred at fields similar to the emitting fields of foreign particles, but 
EDS analysis indicated no foreign elements at arc sites. One should note that some microparticles caused a vacuum arc but weren't completely destroyed by it, and therefore can be studied to confirm the existence of foreign elements and they are listed as foreign particles in the first category. The possible causes of vacuum arcs will be discussed in the following sections.

As shown in Table 5.2, the emitter density is significantly reduced to $28 /\left(6 \times 4.9 \mathrm{~cm}^{2}\right)=0.95 / \mathrm{cm}^{2}$, and reasonably repeatable from sample to sample. Further BCP removal doesn't monotonically reduce the emitter density on the same sample, which indicates that the damage layer is already removed from the surface area, i.e., observed emitter density is independent of BCP removal of additional $\mathrm{Nb}$. Therefore, a fresh new surface was studied each time in subsequent BCPs and treated as if it were a new sample in the statistics. Some SEM pictures of the above emitters are shown in Figure 5.6 along with emitters from the next section.

\subsubsection{Field emission test results after further improvement in sample preparation}

In an effort to further reduce the emitter density and to control the damage extent of vacuum arcs, improvement in sample preparation and in the experimental circuit was made as follows. Several new samples were cut from the same Nb sheet as those listed in Table 5.2, but were made larger so that the field emission scan can be even further away from the edge area, which is prone to have machining contaminants. The previous 1 G $\Omega$ current-limiting resistor was changed to the present $100 \mathrm{G} \Omega$ to further reduce the damage of vacuum arcs and hopefully to preserve the arcing sources for studies. Field emitters subsequently detected at $\sim 140 \mathrm{MV} / \mathrm{m}$ scan are shown in Table 5.3.

As illustrated in Table 5.3, an emitter density of 1-2/sample has been consistently achieved in this series of tests at $\sim 140 \mathrm{MV} / \mathrm{m}$. The average emitter density is $8 /(7 \times$ $\left.4.9 \mathrm{~cm}^{2}\right) \cong 0.23 / \mathrm{cm}^{2}$, reduced by $75 \%$ from the value calculated from Table 5.2 . This 
Table 5.3: Field emission results at $\sim 140 \mathrm{MV} / \mathrm{m}$ from BCP prepared samples after further improvement in machining (the scan area for each sample is $25 \mathrm{~mm}$ dia.)

\begin{tabular}{|c|c|c|}
\hline & Foreign particles & $\begin{array}{c}\text { Emitters destroyed by } \\
\text { vacuum arc } \\
\end{array}$ \\
\hline $\begin{array}{c}\# 75-1 \\
(250 \mu \mathrm{m} \mathrm{BCP}) \\
\end{array}$ & $\begin{array}{c}1 \\
{[35 \mathrm{MV} / \mathrm{m}(\mathrm{Nb}, \mathrm{Fe}, \mathrm{Ti}, \mathrm{Ca})]}\end{array}$ & 0 \\
\hline $\begin{array}{c}\# 75-2 \\
(300 \mu \mathrm{m} \mathrm{BCP}) \\
\end{array}$ & $\begin{array}{c}2 \\
{[99 \mathrm{MV} / \mathrm{m}(\mathrm{Nb}), 103 \mathrm{MV} / \mathrm{m}(\mathrm{Nb})]}\end{array}$ & 0 \\
\hline $\begin{array}{c}\# 72-1 \\
(250 \mu \mathrm{m} \mathrm{BCP}) \\
\end{array}$ & 0 & 1 \\
\hline $\begin{array}{c}\# 72-2 \\
(300 \mu \mathrm{m} \mathrm{BCP})\end{array}$ & 0 & 1 \\
\hline $\begin{array}{c}\# 76-1 \\
(250 \mu \mathrm{m} \mathrm{BCP})\end{array}$ & 0 & 1 \\
\hline $\begin{array}{c}\# 81-1 \\
(200 \mu \mathrm{m} \mathrm{BCP}) \\
\end{array}$ & 0 & 0 \\
\hline $\begin{array}{c}\# 83-1 \\
(200 \mu \mathrm{m} \text { BCP })\end{array}$ & 0 & 2 \\
\hline
\end{tabular}

is the lowest emitter density ever achieved on Nb samples, and it will be discussed in more detail in chapter 9. Only two categories of emitters still remain: foreign particles and emitters destroyed by vacuum arc. The previous category of emitters - features with no foreign elements detected and not particle-like-were not found on this group of samples. One could speculate that they might be the foot prints of contaminant particles that were ultrasonically rinsed off, and the superficial impurity caused the field emission but escaped the detection of EDS.

Just as before, the three foreign particles in Table 5.3 were categorized as such from their appearance and/or the fact that they were rinsed away by a second ultrasonic water rinse. These particles emit over a wide range of electric fields (from 35 to $100 \mathrm{MV} / \mathrm{m}$ ), similar to previous foreign particles. One of the emitters contains $\mathrm{Nb}, \mathrm{Fe}, \mathrm{Ti}$, Ca. Sample \#75-2 is emission free up to $99 \mathrm{MV} / \mathrm{m}$, and sample \#81-1 is emission free up to $140 \mathrm{MV} / \mathrm{m}$. SEM pictures of some of the emitters in Table 5.3 along with selected emitters listed in Table 5.2 are shown in Figure 5.6, and 5.7. 


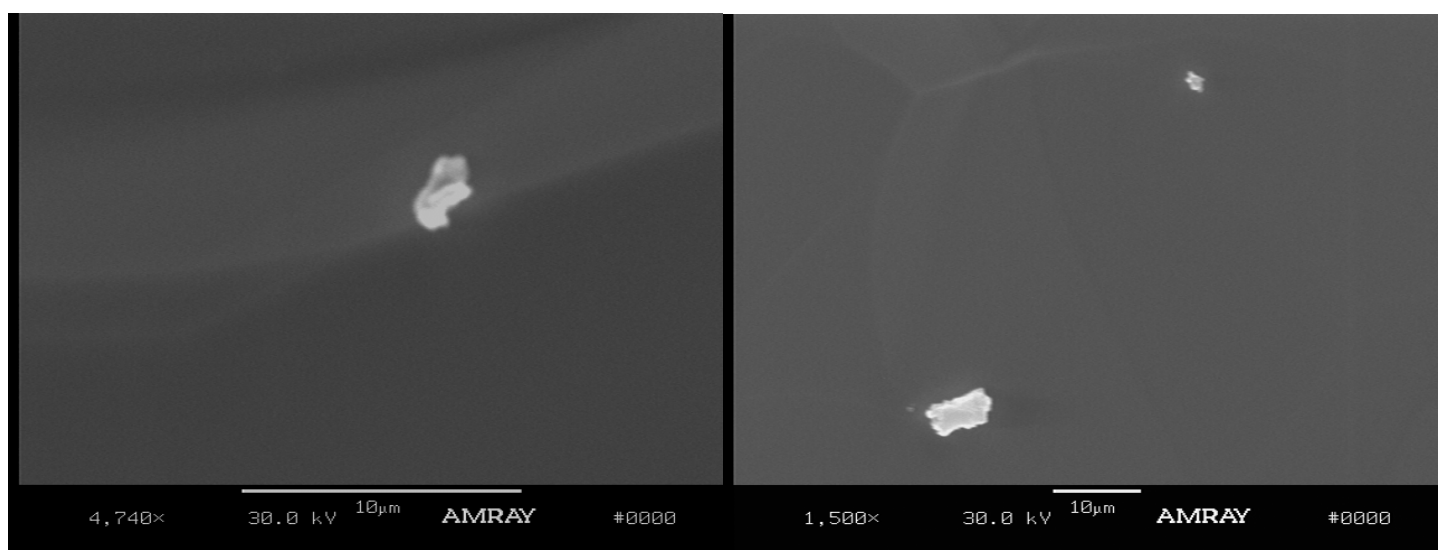

$$
\begin{array}{cc}
\# 65-2 \text { (site } 2), \mathrm{Nb}, \mathrm{Fe}, \mathrm{Cr}, & \# 63-1 \text { (site } 1), \mathrm{Nb}, \mathrm{Fe}, \mathrm{Cr}, \\
E=114 \mathrm{MV} / \mathrm{m} . & E=100 \mathrm{MV} / \mathrm{m} .
\end{array}
$$

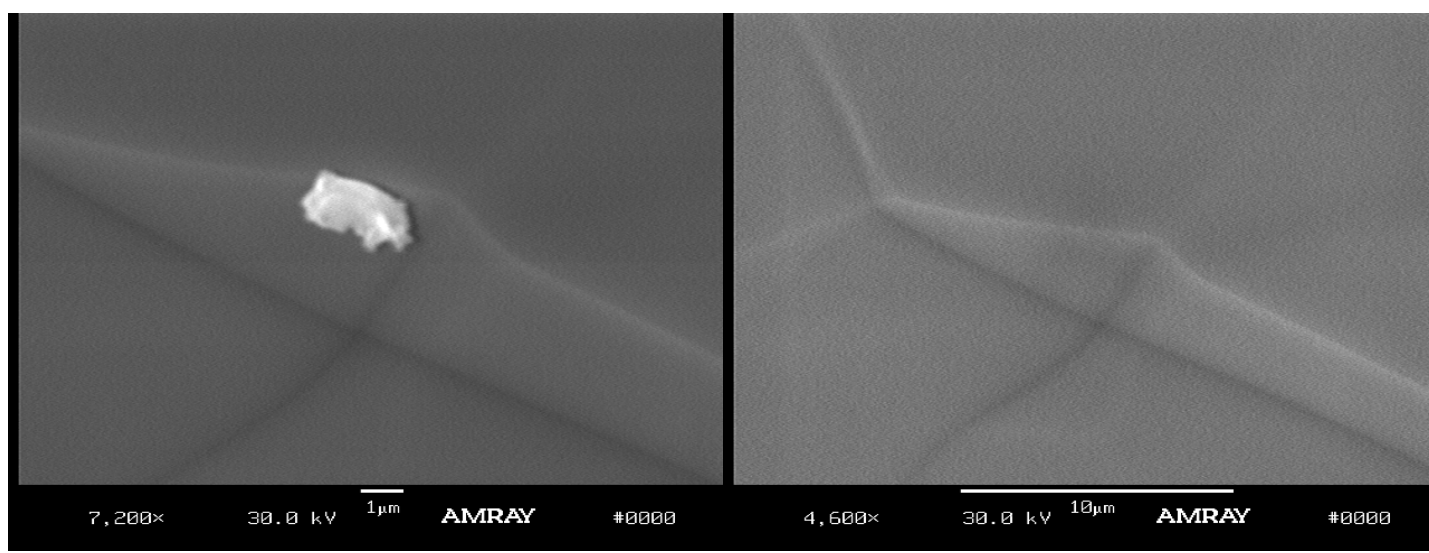
\#66-1 (site 1), Nb,
Same site as left, after an
$E=119 \mathrm{MV} / \mathrm{m}$.
ultrasonic rinse.

Figure 5.6: A selection of SEM pictures of emitters listed in Table 5.2 and 5.3. 


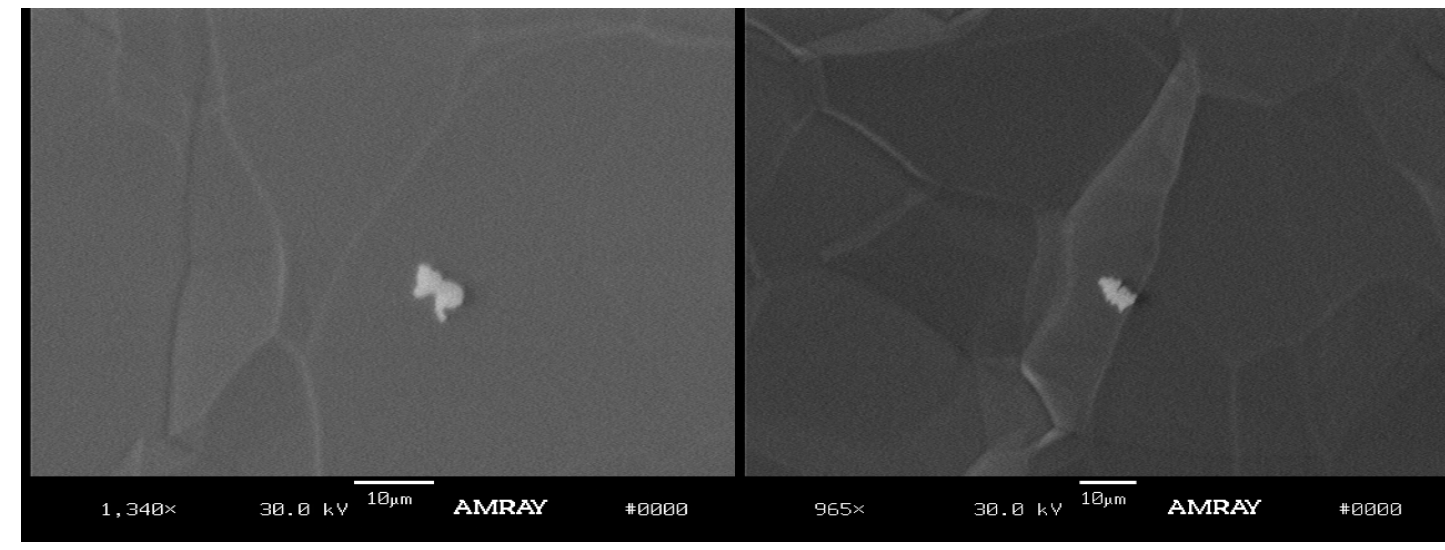

\#75-2 (site 2), Nb, $E=103 \mathrm{MV} / \mathrm{m} . \quad \quad \# 75-2$ (site 1), Nb, $E=99 \mathrm{MV} / \mathrm{m}$.

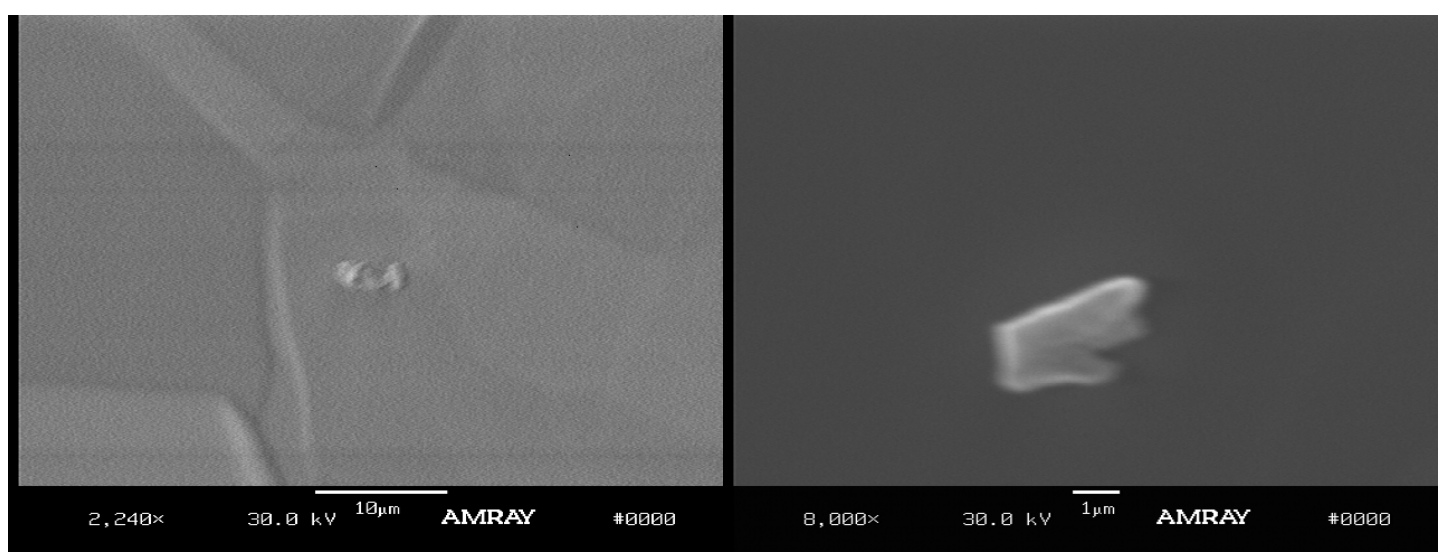

\#75-1 (site 1), Nb, Fe, Ti, Ca, $E=35 \mathrm{MV} / \mathrm{m}$.
\#63-1 (site 3), Nb, Fe, Cr,

$$
E=105 \mathrm{MV} / \mathrm{m} \text {. }
$$

Figure 5.7: A selection of SEM pictures of emitters listed in Table 5.2 and 5.3. 


\subsection{Conclusions and discussion}

Comparing Table 5.3 with Table 5.2, not only were un-destroyed emitters reduced from $14 / 6(\cong 2.33)$ per sample to $3 / 7(=0.43)$ per sample, but also the occurrence of vacuum arcs are further reduced from $14 / 6(\cong 2.33)$ to $5 / 7(\cong 0.71)$ per sample, which indicates that machining contaminant was indeed and may still be the main source of particulate emitters and vacuum arcs, hence may represent the key step in reducing field emission. Whether high pressure water rinse is able to remove the contaminant more thoroughly at the root will give an indication of the origin of the emitters (refer to chapter 7). Other possible sources for the remaining emitters are dust particles from inside the vacuum chamber being stirred up by opening or closing the gate valves, or airborne dust particles in the cleanroom falling onto sample surface during the transfer from the laminar bench to the SEM chamber. A better controlled environment, such as a class 100, or 10 cleanroom may be better suited to achieving further reduction in field emitter density. Whether the foreign particles were caused by operating the gate valve needs to be investigated in possible future experiments.

Vacuum arcs or sparks-transient vacuum breakdown within the gap-are observed with the optical microscope. The mechanisms that initiated vacuum arcs are very complex, some may evolve from an initial field emission while some may not, as described in Latham's book. ${ }^{15}$ Many theories for cathode-initiated arcs are based on the initial heating of the cathode emitter to reach thermal instability, which is unlikely for an intrinsic emitter because of the good thermal contact with the bulk material. In the mean time, the physical transfer of weakly-bound microparticles from cathode to anode or vice versa, due to pure mechanical forces from strong electric field, has been proved by experiments to initiate a breakdown. As described in the previous section, foreign particles were found at the center of some arc sites, which proved that foreign particles can start a breakdown, regardless 
of whether field emission is involved in the process. Furthermore, the fact that the average number of vacuum arcs per sample can be further reduced to $\sim 3 / 10$ of the previously value in Table 5.2 also strengthens the author's view that they are less likely caused by intrinsic emitters, which should largely remain unchanged in number, since all the samples were cut from the same $\mathrm{Nb}$ sheet.

From the results presented above, the following conclusions are drawn:

- Undestroyed emitting sites are found to be foreign microparticles. They are proved mostly likely to be the re-deposited machining contaminant originated from the sample edge.

- No evidence of intrinsic emitters is observed up to $140 \mathrm{MV} / \mathrm{m}$ from chemically etched $\mathrm{Nb}$ samples (scan area: $\sim 64 \mathrm{~cm}^{2}$ ), although more samples can be studied for better statistics.

- Lowest ever emitter density, i.e., $0.23 / \mathrm{cm}^{2}$, and at the highest field of 140 $\mathrm{MV} / \mathrm{m}$, is achieved by chemical etching and ultrasonic rinse alone without heat treatment from each of a series of tests without exception (refer to chapter 9). One sample is emission free up to $99 \mathrm{MV} / \mathrm{m}$, and one sample is emission free up to $140 \mathrm{MV} / \mathrm{m}$. Therefore, reproducible and maximal reduction in $\mathrm{FE}$ for $\mathrm{SRF}$ cavities can be presumably achieved by improving the material machining process, which is often overlooked but shown to be critical by this work, carefully controlling the chemical etching and cleaning process, and stringent particle control afterwards to prevent recontamination.

- To remove remaining emitters would require effort in: a) using more powerful cleaning techniques, e.g., high pressure water rinse, to thoroughly remove machining contaminants at the source. High pressure rinse is a flowing water system, contaminant once dislodged will flow out of the system with water, less likely to re-deposit like in an ultrasonic container (refer to chapter 7). b) investigating the possibility that airborne dust 
particles fall onto the sample surface after rinsing, namely during the transfer to vacuum chamber, and the possibility of dust being stirred up inside the chamber by operating gate valves.

One would notice that one of the experimental objectives, reproducible and minimal field emission density, is achieved without involving the other preparation or treatment methods suggested in the program (refer to chapter 4), which is unexpected. However, the above conclusions are drawn at $140 \mathrm{MV} / \mathrm{m}$ and may only be extrapolated up to a certain field, above which intrinsic emitters, i.e., bulk impurities or grain boundary, may indeed be emitters and would require a much more complex preparation sequence to remove. 


\section{Chapter 6}

\section{Enhanced field emission from electropolished niobium}

\subsection{Electropolishing}

\subsubsection{Motivation: Nb cavity performance improvement by Electropolishing over chemical polishing}

In recent years, researchers at KEK in Japan have discovered the SRF cavity performance improvement by electropolishing (EP) over BCP, i.e., higher acceleration gradient is achieved on cavities. Several other institutions have since formed a collaboration with KEK to investigate the process and have confirmed the discovery with similar results. The statistics of the achieved acceleration gradient on a number of cavities at CERN by BCP and by electropolishing are shown in Figure 6.1.

Refer to Figure 6.2 for the comparison of repeated BCP and electropolishing on the same cavity, as reported by E. Kako and colleagues from KEK in collaboration with researchers from Saclay, Cornell, and DESY. ${ }^{63}$ An EP treatment after BCP can increase the gradient considerably, and a second BCP after EP is observed to degrade the cavity performance, which proves that the difference between BCP and $\mathrm{EP}$ is not due to insufficient amount of removal, but the surface condition itself. Finally a certain amount of EP removal can fully recover the cavity performance. In 


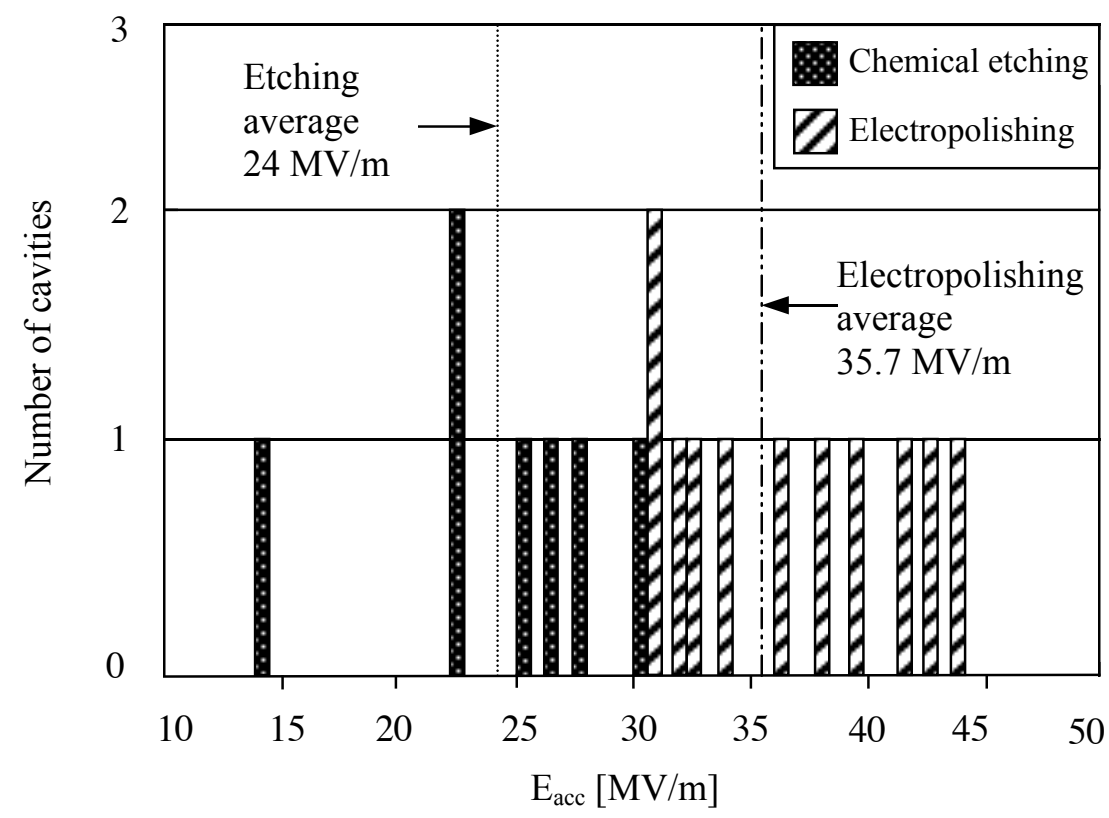

Figure 6.1: The statistics of achieved acceleration gradient on a number of $\mathrm{Nb}$ cavities by chemical etching and by electropolishing at CERN. ${ }^{64}$

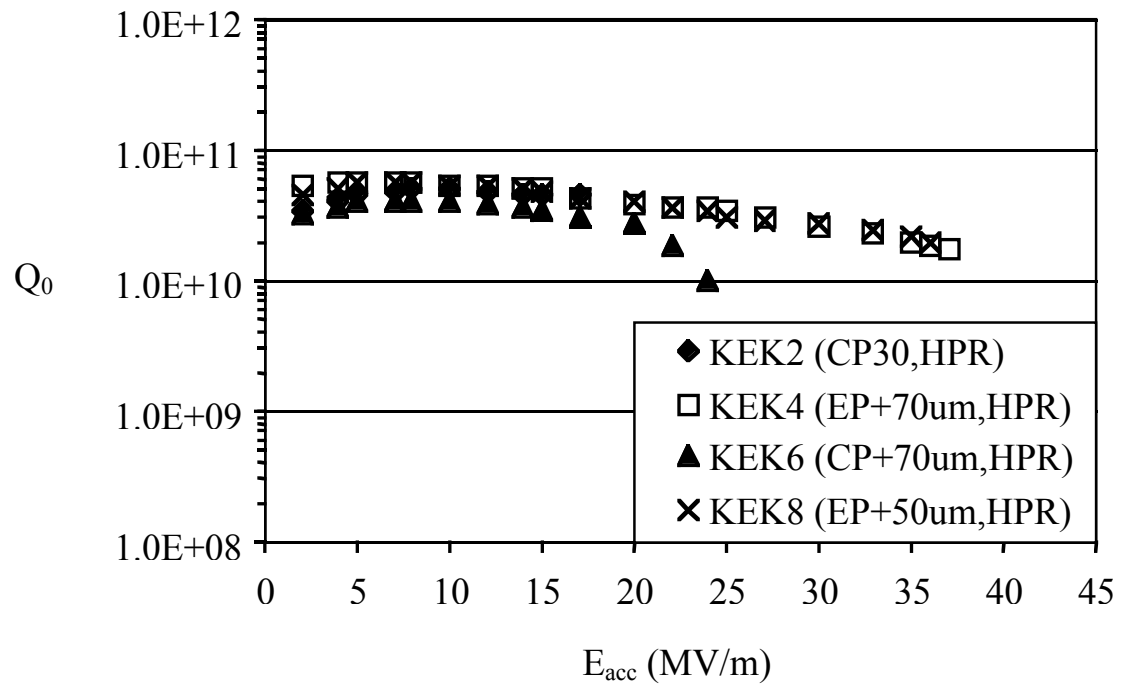

Figure 6.2: The comparison of BCP and EP on the same cavity. ${ }^{63}$ 
both BCP and EP tests, field emission is sometimes observed, especially in multicell cavities, and eventually limit the gradient. Chronologically in parallel with the work on $\mathrm{BCP}$ samples, we studied the field emission performance of electropolished $\mathrm{Nb}$ samples, aiming to investigate whether there is a fundamental difference between the two processes and whether it is due to the different chemistry in removing contaminants or the surface smoothness that makes cleaning/rinsing more effective.

\subsubsection{Electropolishing mechanism}

Electropolishing is also called electrolytic polishing. The specimen to be polished (anode) and a cathode made of a suitable material are immersed in an electrolyte and connected to a battery or power supply. Although the polishing mechanism is not yet completely understood, it is believed that a viscous liquid layer immediately adjacent to the specimen surface is critical in the "smoothing" effect of electropolishing. ${ }^{65}$ The viscous layer, known as polishing film, is a saturated solution of the reaction products between specimen and electrolyte. The polishing film has a greater electrical resistance than the remainder of the electrolyte. As shown in Figure 6.3, at protrusion $\mathrm{A}$ on the specimen surface, the resistance between $\mathrm{A}$ and the top of the polishing film $\mathrm{B}$, is lower than the resistance between valley $\mathrm{C}$ and the top of the polishing film $\mathrm{D}$ because the film is thinner at A. Therefore the current density is higher at $\mathrm{A}$, causing the specimen to dissolve faster there than at $\mathrm{C}$, producing a smooth surface. This "smoothing" mechanism is able to remove irregularities of a micron or larger scale. Moreover, faster diffusion of metal ions and molecules through the thinner polishing film at A may also contribute to the preferential dissolution of peaks.

The other effect of electropolishing, "brightening", is generally attributed to a very thin, partly passivating film directly on the surface of the specimen and following its contours. This thin layer suppresses direct etching by the electrolyte on the surface, 


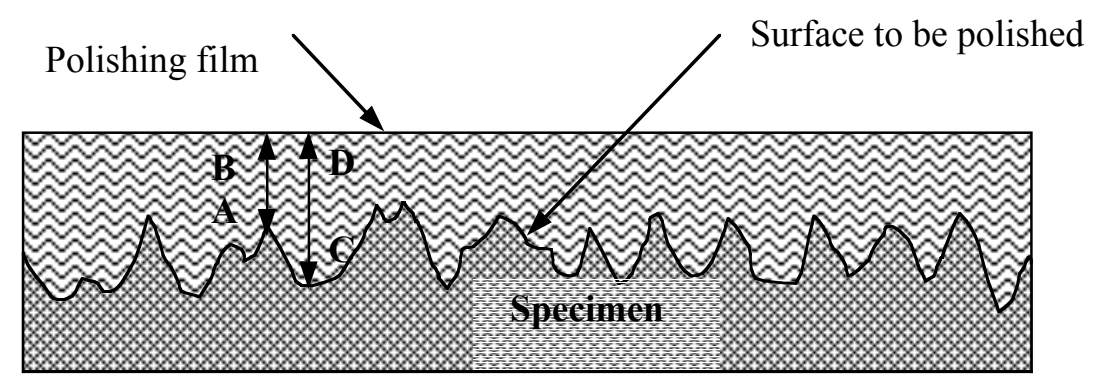

Figure 6.3: Mechanism of electropolishing. ${ }^{65}$

which preferentially occurs at grain boundaries, and enables the elimination of irregularities as small as $\sim 0.01 \mu \mathrm{m}$.

\subsubsection{Electropolishing setup and chemistry for $\mathrm{Nb}$}

The electropolishing method developed by Siemens company is often used for $\mathrm{Nb}$ cavities. ${ }^{66}$ The electrolyte formula consists of $850 \mathrm{ml}$ sulfuric acid (96\%) and $100 \mathrm{ml}$ hydrofluoric acid (40\%). The chemistry involved is: the current oxidizes the $\mathrm{Nb}$ surface; then hydrofluoric acid dissolves the oxides and forms a compound soluble in water, i.e. ${ }^{67,68}$

$$
\begin{gathered}
2 \mathrm{Nb}+5 \mathrm{SO}_{4}{ }^{-2}+5 \mathrm{H}_{2} \mathrm{O} \rightarrow \mathrm{Nb}_{2} \mathrm{O}_{5}+10 \mathrm{H}^{+}+5 \mathrm{SO}_{4}{ }^{-2}+10 \mathrm{e}^{-}, \\
\mathrm{Nb}_{2} \mathrm{O}_{5}+6 \mathrm{HF} \rightarrow \mathrm{H}_{2} \mathrm{NbOF}_{5}(\text { soluble })+\mathrm{NbO}_{2} \mathrm{~F} \bullet 0.5 \mathrm{H}_{2} \mathrm{O} \text { (not soluble) }+1.5 \mathrm{H}_{2} \mathrm{O} \\
\text { and } \mathrm{NbO}_{2} \mathrm{~F} \bullet 0.5 \mathrm{H}_{2} \mathrm{O}+4 \mathrm{HF} \rightarrow \mathrm{H}_{2} \mathrm{NbOF}_{5}(\text { soluble })+1.5 \mathrm{H}_{2} \mathrm{O}
\end{gathered}
$$

The appropriate condition is $10-15 \mathrm{~V}$ constant voltage at room temperature depending on the electrode geometry. The optimum polishing condition is characterized by a current oscillation with decreasing amplitude, which can be explained by the buildup and partial dissolution of the oxide film at the niobium surface. This oxide film overall tends to increase in thickness and results in a decrease of current. Voltage is switched off after the current oscillation has decayed, and in the following several minutes, the oxide film will be dissolved by the hydrofluoric acid in the electrolyte. Furthermore, agitation is employed for the first 
1-2 minutes to speed up the removal of reaction products accumulated near the specimen surface, and to remove gas bubbles to prevent specimen surface pitting. At the end of the cycle, voltage is switched on again and current oscillation recommences. To sum up, each polishing cycle is divided into three regimes: polishing (voltage on, stirring off); agitation (voltage off, stirring on); and resting (voltage off, stirring off). Each cycle is $\sim 5$ minutes, and the polishing rate is $\sim 2$ $\mu \mathrm{m} /$ cycle.

The set up for electropolishing $\mathrm{Nb}$ sample is schematically shown in Figure 6.4. The cathode is made of aluminum, which is inert in the electrolyte being used, and an anode fixture made of $\mathrm{Nb}$ is used to hold the $\mathrm{Nb}$ sample in place. At the aluminum cathode, $\mathrm{H}_{2}$ is formed and gassed out. One of the disadvantages of electropolishing is that it does not attack non-metallic contaminants on the specimen surface.

The resulting $\mathrm{Nb}$ surface is very smooth and shiny compared to the surface conditions obtained by BCP. A comparison of their SEM pictures is shown in Figure 6.5 .

\subsection{Field emission results from electropolished niobium}

After the tests listed in Table 5.2, sample \#65 and \#63 were BCP etched to remove another $80 \mu \mathrm{m}$, then electropolished to remove $\sim 40 \mu \mathrm{m}$. Sample \#61 of the same size was chemically etched by $\mathrm{BCP}$ to remove $330 \mu \mathrm{m}$ in total, then also electropolished to remove an additional $40 \mu \mathrm{m}$. Subsequently, the samples were ultrasonic cleaned in DI water and then briefly rinsed in methanol followed by laminar air drying in the cleanroom. The field emission scan results at $\sim 140 \mathrm{MV} / \mathrm{m}$ are shown in Table 6.1. 


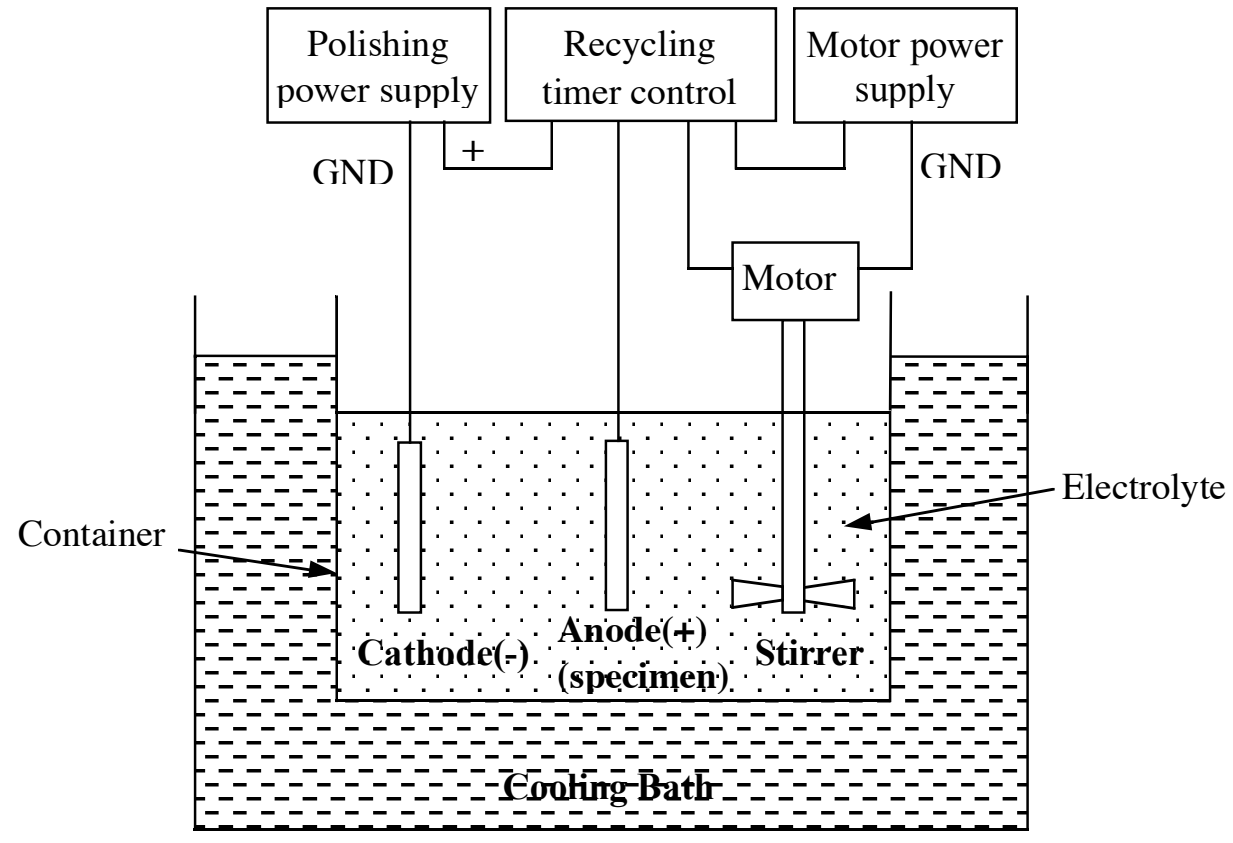

Figure 6.4: The schematic setup for electropolishing of Nb.

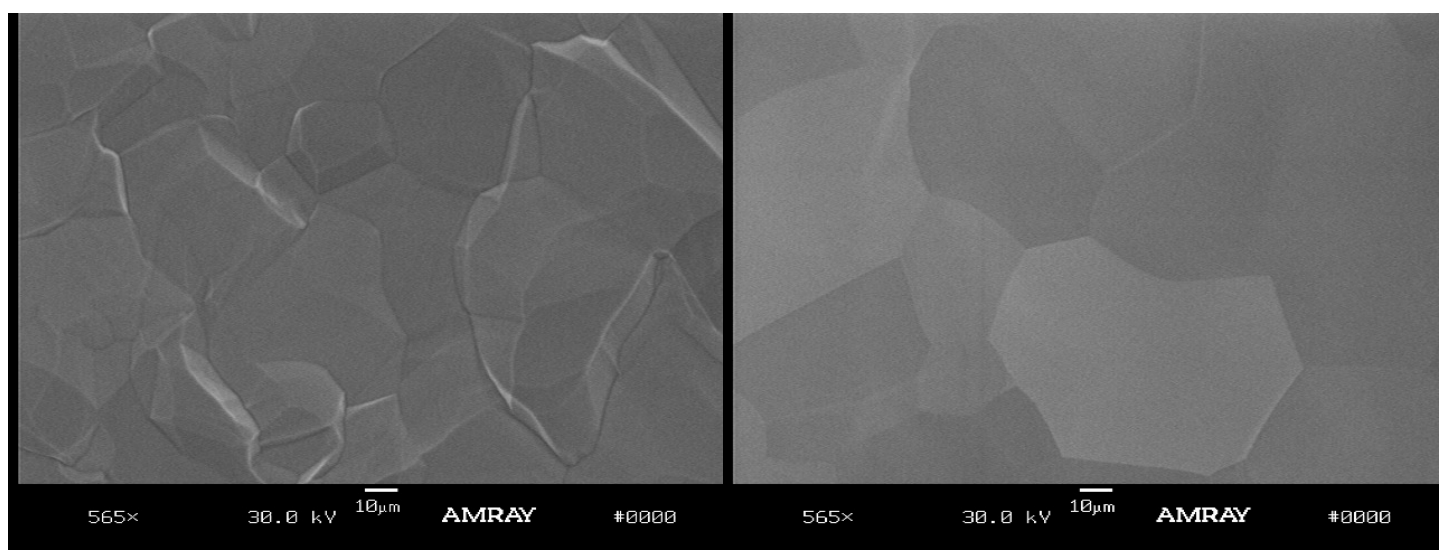

Figure 6.5: Comparison of BCP etched and electropolished Nb sample surface. 
Table 6.1: Field emission results from electropolished Nb samples (scanning field $\sim 140 \mathrm{MV} / \mathrm{m}$, scan area per sample is $25 \mathrm{~mm}$ dia.)

\begin{tabular}{ccc}
\hline & Foreign particles & $\begin{array}{c}\text { Emitters destroyed by } \\
\text { vacuum arc }\end{array}$ \\
\hline $\begin{array}{c}\text { \#65-4 }(440 \mu \mathrm{m} \text { total BCP } \\
+40 \mu \mathrm{m} \mathrm{EP})\end{array}$ & $\begin{array}{c}1 \\
\text { (105 MV } / \mathrm{m}(\mathrm{Nb}, \mathrm{Fe}, \mathrm{Cr})]\end{array}$ \\
\hline $\begin{array}{c}\text { \#61-1 }(330 \mu \mathrm{m} \text { total BCP } \\
+40 \mu \mathrm{m} \mathrm{EP})\end{array}$ & 0 & 1 \\
\hline $\begin{array}{c}\# 63-3(410 \mu \mathrm{m} \text { total BCP } \\
+40 \mu \mathrm{m} \mathrm{EP})\end{array}$ & 0 & 4 \\
\hline
\end{tabular}

The emitter categories are the same as for BCP samples: foreign particles and emitters destroyed by vacuum arc. No difference caused by chemistry is observed. The only foreign particulate emitter in these tests, which caused a vacuum arc but wasn't destroyed by it, contains $\mathrm{Nb}, \mathrm{Fe}$, and Cr. As discussed before, it could be redeposited contaminant from stainless steel machining tools. Its SEM picture is shown in Figure 6.6 (the nearby craters are caused by vacuum arc) along with a picture of an arc site. An emission free surface is achieved on \#65-4 up to 105 $\mathrm{MV} / \mathrm{m}$.

The average emitter density of $6 /\left(3 \times 4.9 \mathrm{~cm}^{2}\right)=0.4 / \mathrm{cm}^{2}$, is lower than corresponding chemically etched samples in Table 5.2 , i.e., $0.95 / \mathrm{cm}^{2}$. However, the low base number of emitters makes it difficult to statistically state that the smoother surface does make particle removal more effective. To truly compare the cleaning effectiveness, one could introduce an equal and large number of foreign particles on $\mathrm{BCP}$ and $\mathrm{EP}$ surfaces that are initially nearly particle free, then compare the particles left after cleaning. 


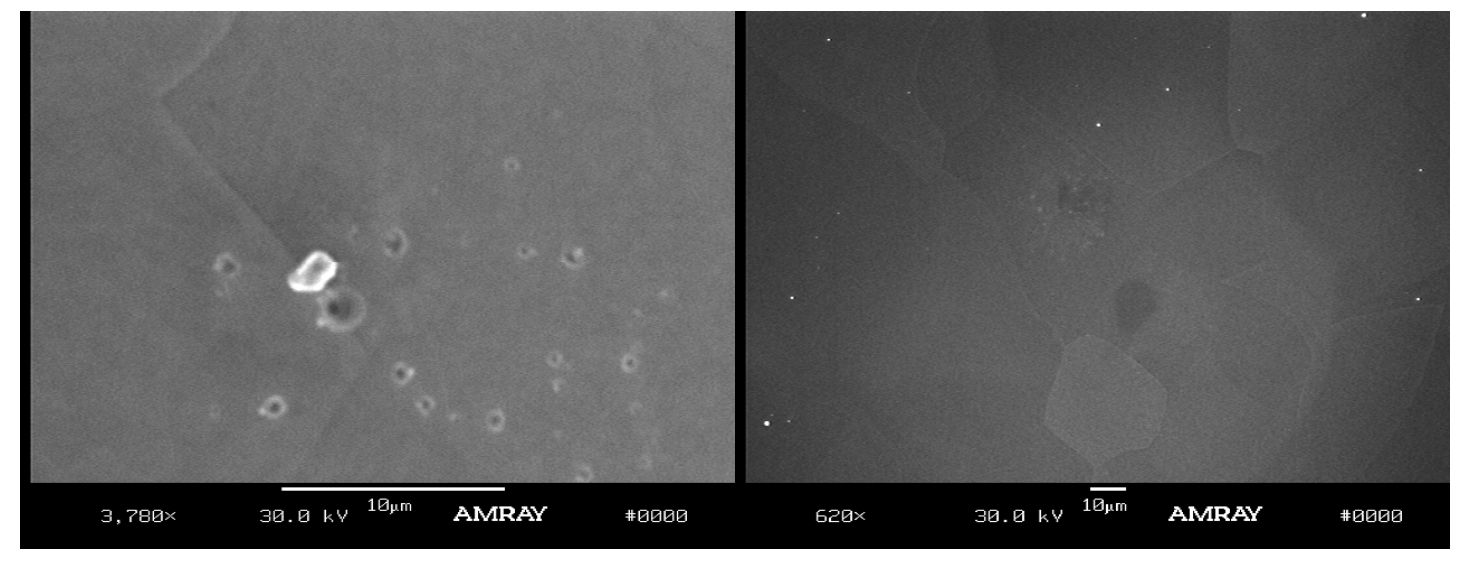

Figure 6.6: A foreign particle emitter from sample \#65-1 (left, the craters are caused by vacuum arc, the particle contains $\mathrm{Nb}, \mathrm{Fe}, \mathrm{Cr}, E=105 \mathrm{MV} / \mathrm{m}$ ) and an arc site from sample \#63-1 (right, the small particles are generated by vacuum arc) from electropolished $\mathrm{Nb}$ samples.

\subsection{Comparison between chemically etched and electropolished $\mathrm{Nb}$ samples}

From the results presented above, the following conclusions are drawn:

- No difference is observed in the categories of field emission sources from electropolished $\mathrm{Nb}$ samples up to $140 \mathrm{MV} / \mathrm{m}$ compared to BCP samples. Namely, the different chemistry didn't produce different field emission sources.

- No intrinsic emitters have been observed up to $140 \mathrm{MV} / \mathrm{m}$ from electropolished samples either (scan area: $\sim 15 \mathrm{~cm}^{2}$ ). More samples can be studied for better statistics.

- Electropolished surfaces result in $\sim 1 / 2$ of the emitter density for corresponding BCP surfaces. However, the low base number of emitters make it difficult to statistically compare the cleaning effectiveness on the two types of surfaces. 
- The only foreign particulate emitter contained $\mathrm{Nb}, \mathrm{Fe}, \mathrm{Cr}$, consistent with findings from $\mathrm{BCP}$ etched samples, which indicates that the emitters are likely the same origin-contaminant from machining tools. Other possibilities are dust from inside the vacuum chamber or airborne dust particles that fell onto sample surfaces during the transfer in the cleanroom. To achieve further reduction in $\mathrm{Nb}$ samples or cavities would require additional effort in these areas. 


\section{Chapter 7}

\section{Effect of different drying processes}

\subsection{Experimental description}

\subsubsection{Motivation and objectives of the study}

The cleaning technique, clean assembly, and drying process to avoid recontamination have all proven to be critical in achieving high gradient. So far the sample preparation process has followed the procedures most commonly used for today's cavities except for the high pressure water rinse and the drying process. Although the emitter density has consistently dropped to near zero by BCP or EP and ultrasonic cleaning only, whether or not the high pressure rinse will reduce the density even further could provide evidence on the origin of the remainder of the emitters, i.e., before or after the sample cleaning. High pressure rinse has proven to be a powerful tool in cleaning cavities. Its setup will be introduced in the next section. As for the drying processes, there are different approaches at various labs. Cornell uses warm filtered nitrogen gas to blow dry cavities. At KEK, the wet cavity is assembled and dried by applying heat while pumping out the vapor. At Jefferson Lab, cavities are rinsed with ultrapure methanol or ethanol, then let to dry in a Class 10 cleanroom. Due to the safety hazard involved in handling large volumes of methanol or ethanol, this step is now often skipped and cavities are left in the high pressure rinse cabinet or a Class 10 area in a Class 100 cleanroom to dry naturally. The duration of this drying time spans from several hours to a couple of 
days. In some multicell cavities severe EFE were encountered, and it has been speculated that this drying practice may have created new emitters because water is known to attract dust or nucleate on a dust grain, react with residual chemicals, produce acids (e.g., by dissolving carbon dioxide from the air) and cause corrosion, which all could become field emitters. The extended exposure to airborne dust adds to the risk of re-contamination. Therefore, it is important to investigate and compare the "slow drying" with the "quick drying" (with methanol rinse) and to provide guidance on this procedure. The comparative experiments integrating high pressure rinse were performed and will be described in this chapter.

\subsubsection{High pressure ultra pure water rinse}

High pressure ultra pure water rinse has become an indispensable procedure in cleaning cavities in the past several years. Its effectiveness is not limited to cleaning particles that may be difficult to remove by other means, e.g., ultrasonic, it has also proven to be powerful enough to smooth out sharp protrusions caused by scratches on surfaces as well. The setup for high pressure rinse is shown schematically in Figure 7.1. High pressure ( $\sim 80$ bar) water is sprayed through a nozzle to scan across all parts of the cavity's inner surface and dislodge and sweep away microparticles. The resistivity of the water is close to theoretically pure $(17.5 \mathrm{M} \Omega-\mathrm{cm})$. The water is filtered to eliminate the introduction of microparticles. Particles removed from the surface flow out of the system with the water, hence they are less likely to re-deposit to the surfaces.

\subsubsection{Experimental procedures}

Three $\mathrm{Nb}$ samples were $\mathrm{BCP}$ prepared, ultrasonic cleaned, methanol rinsed and laminar dried in the same way as before, and then scanned for field emitters at 140 $\mathrm{MV} / \mathrm{m}$. The field emission results are labeled as "ultrasonic rinse", as shown in

Figure 7.2. In order to better simulate the resulting cavity surface condition, each 


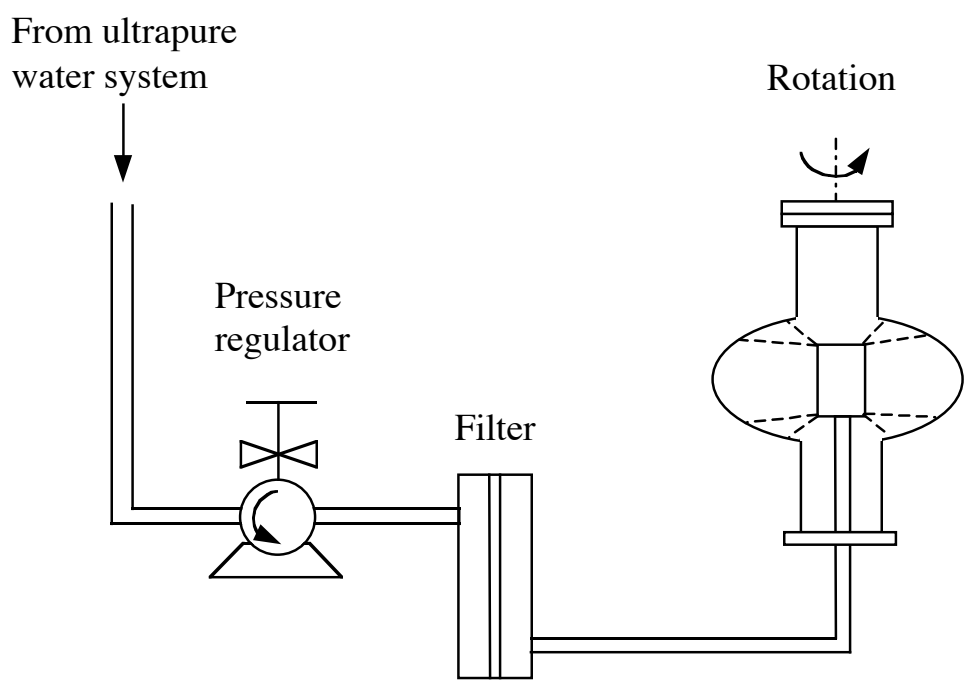

Figure 7.1: The setup for high pressure water rinse.

sample was then mounted inside of a mock cavity, which is similar in size and shape to a real single-cell cavity, for high pressure rinse. The mock cavity has a detachable side panel for mounting one sample at a time. After the high pressure rinse, the mock cavity was taken back to the R\&D cleanroom, where the SFEM is located, with both flanges covered up by clean plastic caps to prevent re-contamination during the transfer. The sample was then dissembled from the mock cavity and briefly methanol rinsed to displace water before being dried on a laminar flow bench. This second field emission scan is labeled as "quick drying" in Figure 7.2. In the final step, the same samples were high pressure rinsed again, then taken to Class 10 cleanroom, where some cavities were naturally dried, and left there with flanges open for 3-4 days. Finally, the mock cavity was transferred to the R\&D cleanroom, the sample was detached and loaded into the apparatus for field emission scan. The last scan is labeled as "slow drying" in Figure 7.2. All the handling and transferring procedures are the same for "quick drying" and "slow drying" except the drying process, therefore the difference in the field emission performance should reflect the difference caused by the drying processes. 

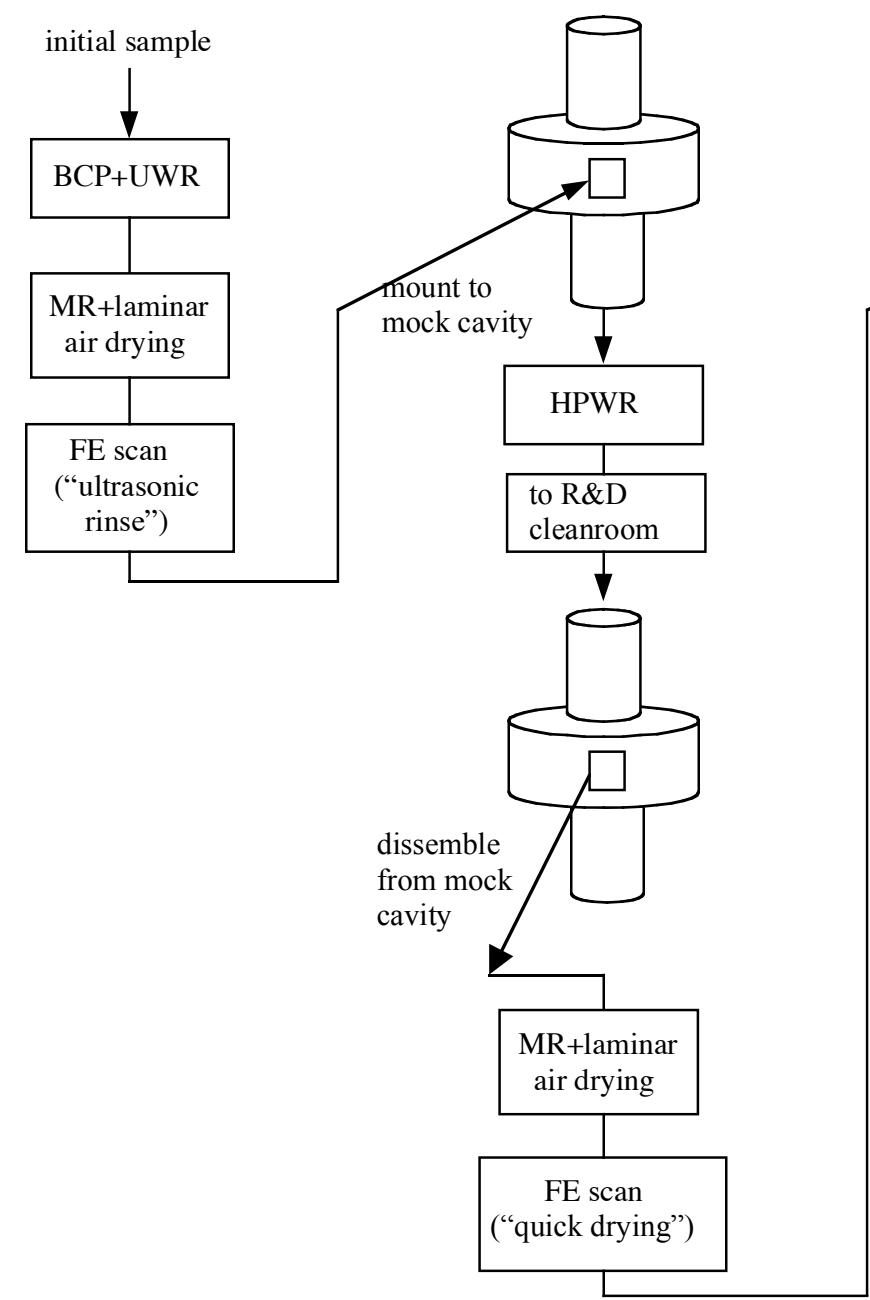

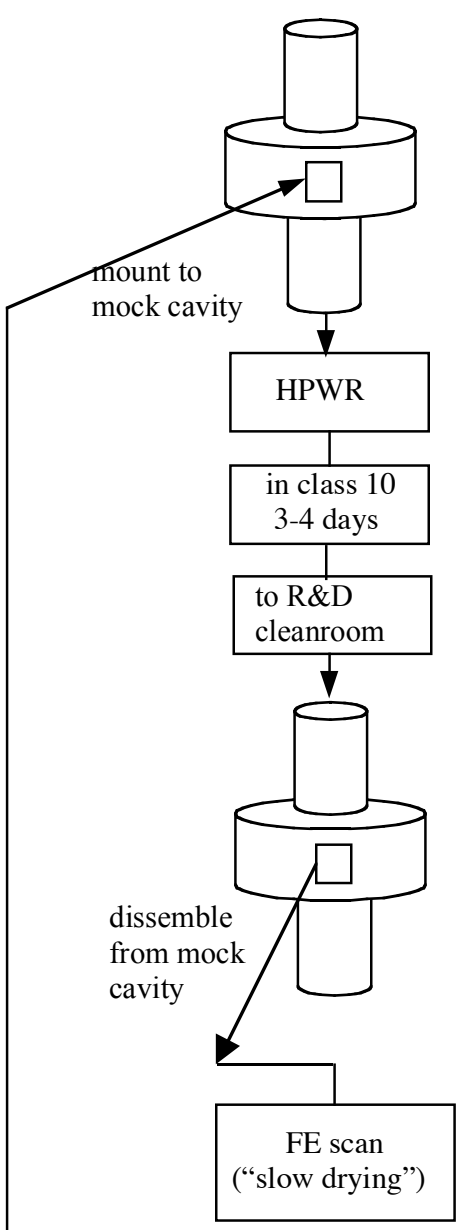

Figure 7.2: The experimental procedures for the comparison of drying processes. 


\subsection{Field emission results}

The results from each stage are shown in Figure 7.3. Sample \#71, 72 and 84 were initially BCP removed $300 \mu \mathrm{m}, 300 \mu \mathrm{m}$ and $200 \mu \mathrm{m}$ respectively before the experiment.

From tests on samples \#71 and 72, there were only arc occurrences, with their locations shown in Figure 7.3, but no undestroyed emitters were left afterwards. The group of four arcs on sample \#72 "quick drying" were caused by the arc particles generated from "ultrasonic rinse" scan (note the location of the arcs for the two scans), hence this group of arcs were not counted as new emitters. From Figure 7.3, one new emitter/arc per sample appeared after the "slow drying" in the Class 10 area for $\sim 67$ hours on sample \#71 and 72, and four new emitters/arc appeared after "slow drying" in Class 10 for 90 hours on sample \#84. Comparing "ultrasonic" and "quick drying" results, the transfer from HPWR stand to the R\&D cleanroom introduced $\leq 0-2$ emitters/sample. Therefore, no significant degradation in field emission is observed by "slow drying" in a Class 10 area, as long as the time duration is kept as short as possible. One should also note that on sample \#71, an emission free surface is achieved up to $140 \mathrm{MV} / \mathrm{m}$ with HPWR and quick drying. On sample \#84, the emitter from "ultrasonic rinse" (emitting field: $93 \mathrm{MV} / \mathrm{m}$ ) and one of the emitters from "slow drying" (emitting field: $104 \mathrm{MV} / \mathrm{m}$ ) don't have any discernible features. The other emitter from "quick drying" (119 MV/m) and one from "slow drying" (108 MV/m) were shown in Figure 7.4. EDS didn't show any foreign elements. The rest of emitting sites on \#84 all caused vacuum arc.

\subsection{Discussion}

We conclude from above experiments that a significant degradation in field emission performance should not be expected by "slow drying" in Class 10, provided the time duration can be controlled to be as short as possible. The risk for degradation is 

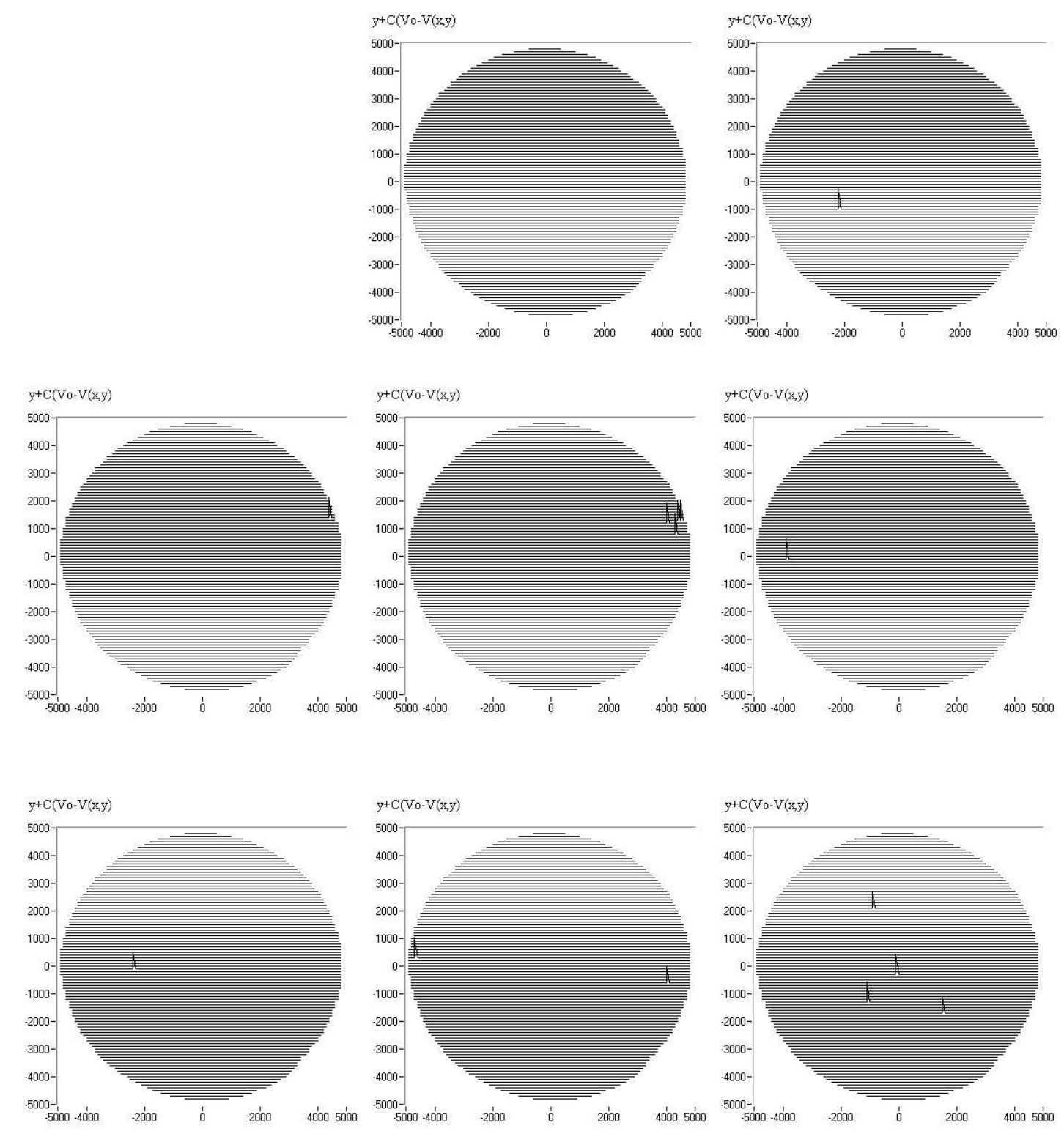

Figure 7.3: Field emission results for the comparison of drying processes. Sample \#71, \#72 and \#84 is in row 1, 2 and 3 respectively. The first column is for "ultrasonic rinse", the second is for "quick drying", and the third is for "slow drying”. 


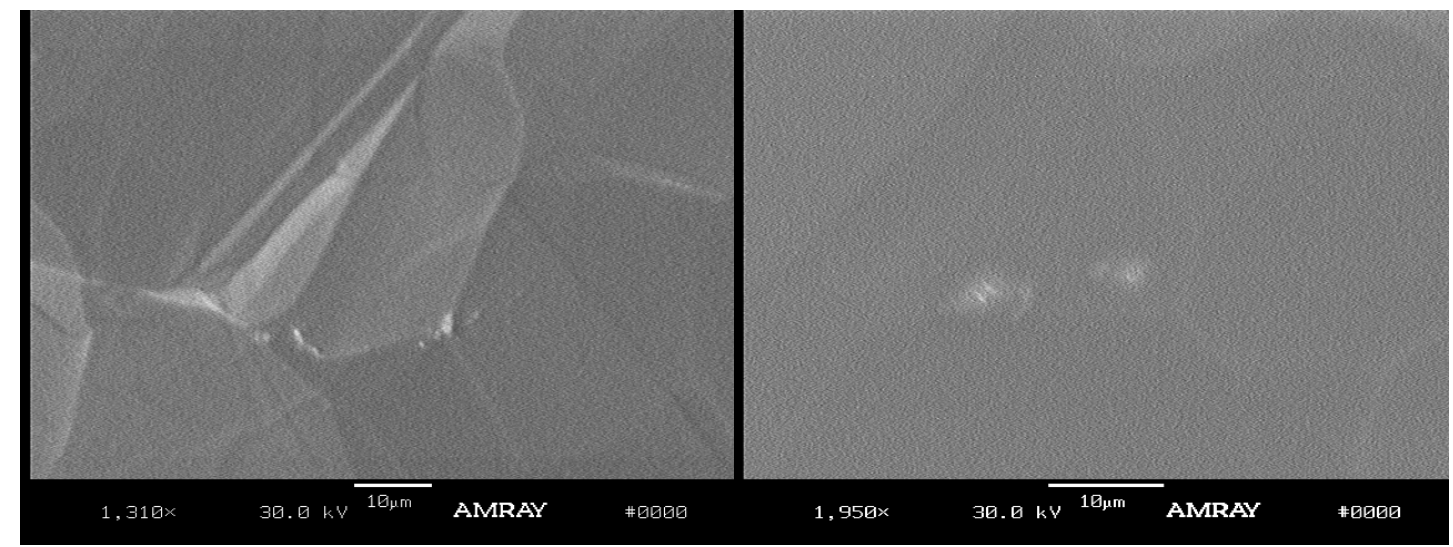

Figure 7.4: SEM pictures of emitters from sample \#84, left is after "quick drying", right is after "slow drying". EDS didn't detect any foreign elements.

mainly from extended exposure to airborne dust particles. No evidence is observed that new emitters are created by reaction of water with material or residual chemicals that emit at $\leq 140 \mathrm{MV} / \mathrm{m}$. However one can not assume that "slow drying" is inherently comparable to "quick drying". In a less-than-ideally controlled environment, for instance Class 1000 or even Class 100, the outcome could be considerably different due to airborne dust or uncontrolled atmospheric chemicals. Comparing "ultrasonic rinse" and "quick drying" from sample \#72 and \#84, the emitter density is roughly comparable and similar to previous samples in Table 5.3, i.e., the emitter density didn't drop further by HPWR. Therefore, it is likely that the remaining emitters are airborne dust falling onto the surface during transfer. Although no significant advantage of HPWR over ultrasonic has been observed on these samples, the low emitter density makes the comparison impossible or at least less-than-conclusive. A more conclusive comparison of the cleaning effectiveness of ultrasonic and HPWR, on BCP or electropolished samples, would be a test with a 
large number of artificial particles intentionally deposited on sample surfaces subject to the cleaning and examined for remaining particles. 


\section{Chapter 8}

\section{Enhanced field emission from energetically deposited niobium thin film}

\subsection{Energetically deposited niobium film}

$\mathrm{Nb}$ thin films deposited on $\mathrm{Cu}$ present a possible alternative to the customary bulk $\mathrm{Nb}$ in superconducting cavities. An energetic $\mathrm{Nb}$ deposition system has been developed by Genfa Wu at TJNAF ${ }^{69}$ The EFE performance of the Nb film itself, as well as possible surface condition requirements for the $\mathrm{Cu}$ substrate need to be investigated to evaluate the feasibility of its application to future accelerator cavities.

The basic concept of energetic deposition is that if the deposited atoms gain more mobility (energy), they will diffuse quickly on the surface and fill surface voids, hence columnar structures are less likely to form, as is shown in Figure 8.1. The energetic deposition system uses energy-controllable metal ions for the deposition, a process also called energetic condensation. The other advantages of this system include: high vacuum (less impurities), no working gas (less impurities introduced by gas, no trapping of working gas in the film, which may cause intrinsic defects, etc.). 


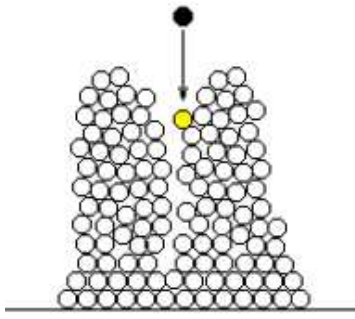

a

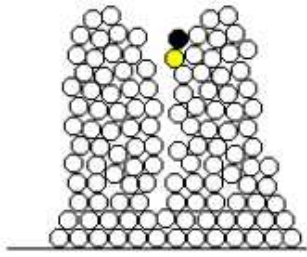

b

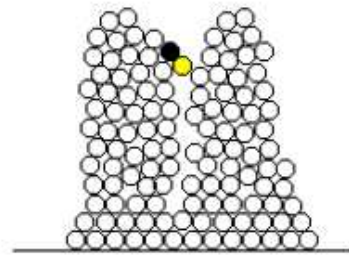

C

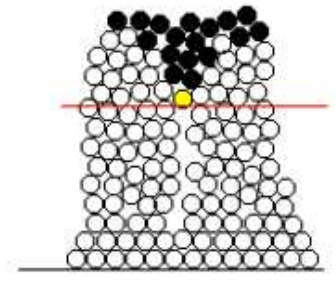

d

Figure 8.1: Illustration of surface atom nucleation: column forming in relation to atom mobility.

The system layout is illustrated in Figure 8.2. Neutral niobium flux is generated by e-beam evaporation, then is ionized by Electron-Cyclotron-Resonance (ECR) inside a waveguide resonator. Namely, the system use microwave power to create pure $\mathrm{Nb}$ plasma, from which energetic $\mathrm{Nb}$ ion flux can then be extracted for direct deposition on a $\mathrm{Cu}$ substrate.

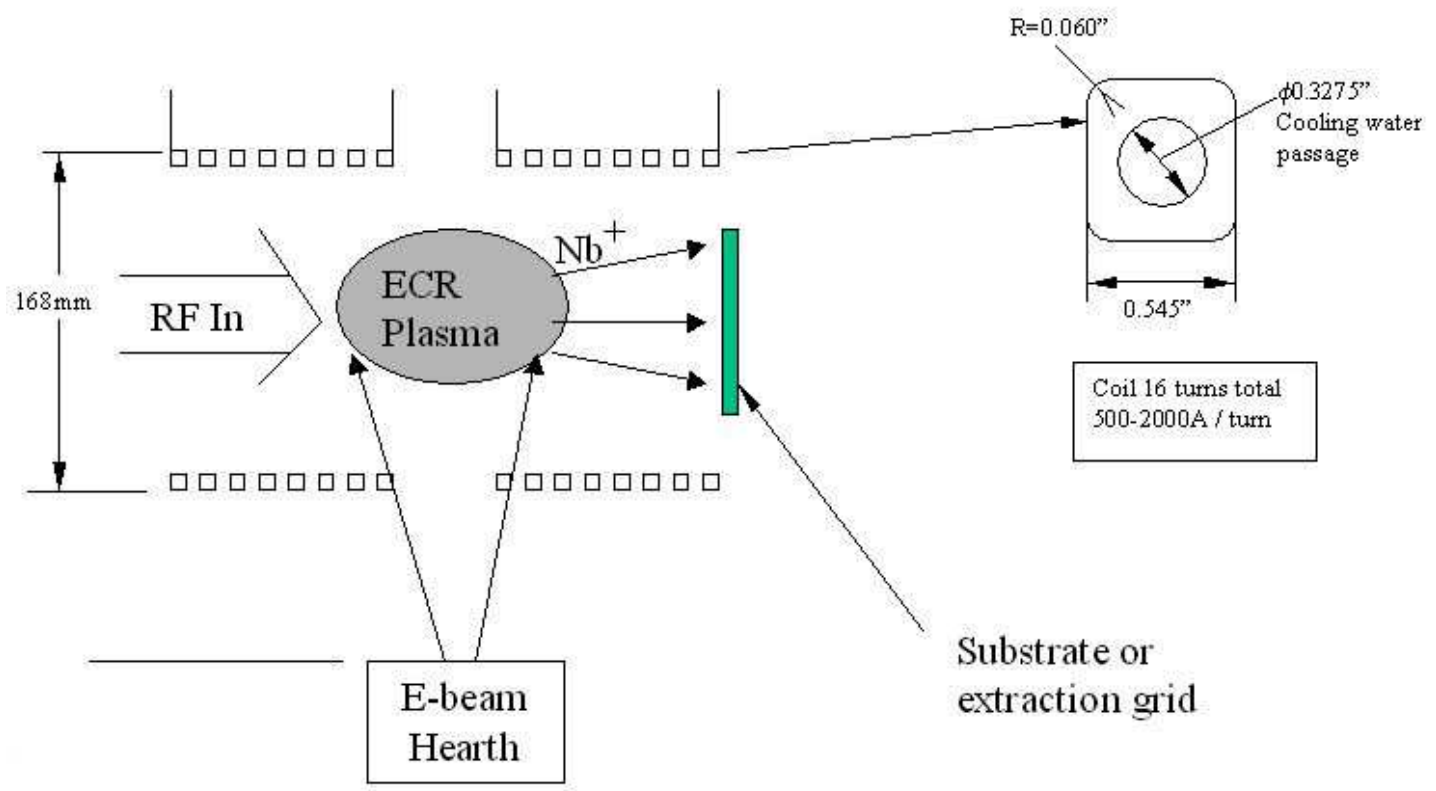

Figure 8.2: Illustration of the energetic deposition system by ECR in vacuum. 


\subsection{Enhanced field emission studies on energetically deposited $\mathrm{Nb}$ thin film}

One of the first deposited $\mathrm{Nb}$ films $(\sim 1 \mu \mathrm{m}$ thick $)$ on $\mathrm{Cu}$ substrate produced by the energetic deposition system was scanned for field emission at $140 \mathrm{MV} / \mathrm{m}$. The $\mathrm{Cu}$ substrate was electropolished to remove $200 \mu \mathrm{m}$ before the deposition. The sample was transferred to the $\mathrm{R} \& \mathrm{D}$ cleanroom in a container after deposition and pasted on top of an old pure $\mathrm{Nb}$ sample in order to seat in the three-post sample holder. Less than half of the sample surface area was scanned, showing $\sim 20$ arcs and $\sim 20$ emitters with emitting field ranging from $\sim 30$ to $140 \mathrm{MV} / \mathrm{m}$. Some emitting sites were found to contain $\mathrm{Cu}, \mathrm{Fe}, \mathrm{Ni}, \mathrm{Cr}$, such as the one shown in Figure 8.3. Many particles with various composition, for example, $\mathrm{Fe}, \mathrm{Cr}, \mathrm{Mn}, \mathrm{Si}, \mathrm{Al}, \mathrm{Mg}, \mathrm{K}$, were found on the surface, likely dust particles introduced during transfer. The sample was observed from the long distance microscope to have many round recessions, roughly 10-20 $\mu \mathrm{m}$ in diameter, and some big pits of $\sim 50 \mu \mathrm{m}$ in diameter. Considering the thickness of the film, these large features are most likely from the $\mathrm{Cu}$ substrate, which is confirmed by off-line optical microscope observations since these features are also present on the $\mathrm{Cu}$ substrate where there is no $\mathrm{Nb}$ film. In some locations, there is no emission or arcing up to $140 \mathrm{MV} / \mathrm{m}$ in a local $\mathrm{mm}^{2}$ area, i.e., no evidence of intrinsic emission was observed from the film.

Clearly, in order for the film to achieve desirable field emission performance at high field or for other aspects of film quality, the substrate polishing technique needs to be studied and modified to prevent pitting and produce a smooth and uniform surface. Ultrasonic cleaning or high pressure rinse will be needed to remove dust particles introduced from handling. Whether the film can tolerate the cleaning without degrading the film field emission quality, crystal structure, critical field, etc. can be tested in future experiments. 


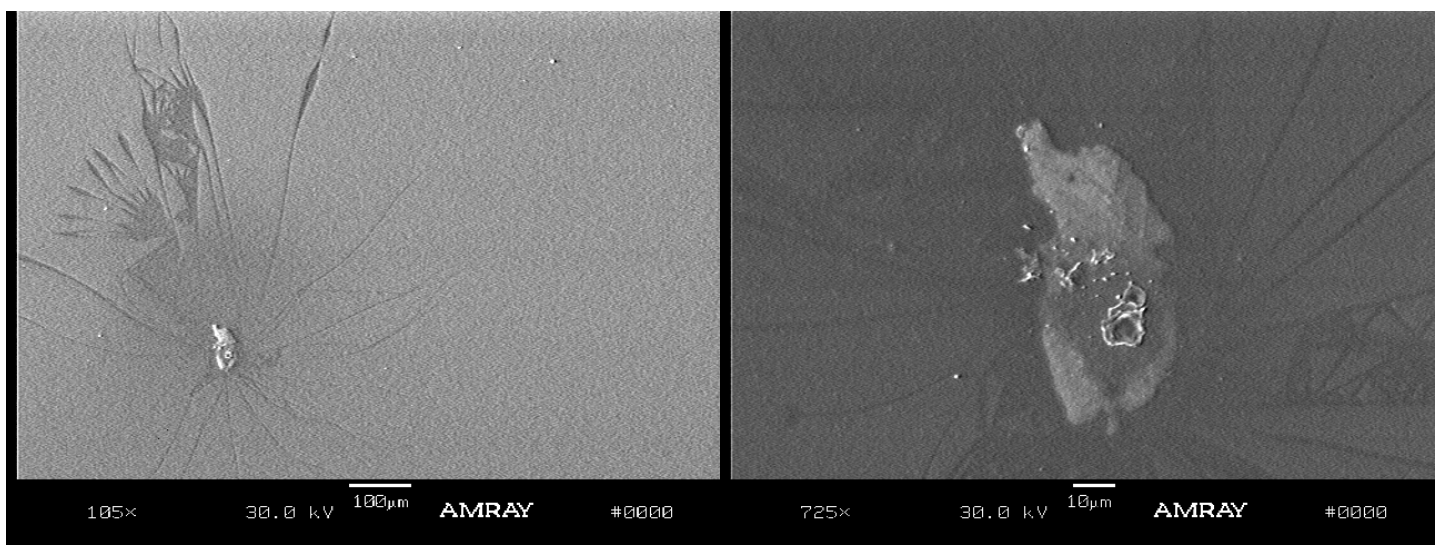

Figure 8.3: One emitter located on energetically deposited $\mathrm{Nb}$ thin film. The pictures are from the same site, but at different magnification. The crater was generated by vacuum arc. It contains $\mathrm{Cu}, \mathrm{Fe}, \mathrm{Ni}$. Cr. Note the starburst and tracking caused by the vacuum arc. 


\section{Chapter 9}

\section{Summary and discussion}

\subsection{Discussion on field emission physical mechanism}

\subsubsection{Various models-supporting and refuting evidence}

All located emitters appeared to be conducting under SEM, and the majority of them contain foreign elements. All emitters are found to be in electrical contact with the $\mathrm{Nb}$ sample (no charging in SEM), which is in favor of the geometrical enhancement model. However, many emitters lack the necessary sharp protrusion for the emission to occur. Tiny round melted particles of $\mathrm{Nb}$ and $\mathrm{W}$ (from anode) generated from vacuum arcing occasionally can become new emitters, even though often at $\sim 100 \mathrm{MV} / \mathrm{m}$ or higher, the discrepancy in geometrical factor is still not negligible, thus undermining the possibility that the geometrical enhancement model represents the whole story. Although all emitters identified in this work were found to be conducting, it could be that our sample preparation and handling environment didn't favor the presence of insulating particles. We occasionally observed non-emitting conducting particles with appearances that are not too different from the emitting ones, but since there were few particles on the surfaces to begin with, we can not comment on the percentage of the particles that did not emit.

The summary of relevant findings by several institutions are listed in Table 9.1. The results of this work present additional hints on the physical mechanism of field 
Table 9.1: Relevant findings from various institutions. (Listed under University of Aston is Latham and co-workers findings $\left.{ }^{15}\right)$. $\sqrt{ }$ : yes, $\mathrm{x}$ : no.

\begin{tabular}{|c|c|c|c|c|c|c|}
\hline $\begin{array}{l}\text { Discoveries \& } \\
\text { inferences }\end{array}$ & $\begin{array}{l}\text { U. } \\
\text { Geneva } \\
\text { (dc) }\end{array}$ & $\begin{array}{c}\text { Cornell } \\
\text { U. } \\
(\mathrm{rf})\end{array}$ & $\begin{array}{l}\text { SACLAY } \\
\& \\
\text { ORSAY } \\
\text { (dc) }\end{array}$ & $\begin{array}{c}\text { U. } \\
\text { Wupper } \\
\text {-tal } \\
\text { (dc) }\end{array}$ & $\begin{array}{l}\text { U. } \\
\text { Aston } \\
\text { (dc) }\end{array}$ & $\begin{array}{l}\text { Jlab } \\
(\mathrm{dc})\end{array}$ \\
\hline $\begin{array}{l}\text { Natural emitters are } \\
\text { generally micron or } \\
\text { submicron particles \& } \\
\text { geometrical defects. }\end{array}$ & $\sqrt{ }$ & $\sqrt{ }$ & $\sqrt{ }$ & $\sqrt{ }$ & $\sqrt{ }$ & $\sqrt{ }$ \\
\hline $\begin{array}{l}\text { Most particles on } \\
\text { surface do not emit. }\end{array}$ & $\sqrt{ }$ & & $\sqrt{ }$ & $\sqrt{ }$ & & $\begin{array}{l}\text { Some } \\
\text { do not. }\end{array}$ \\
\hline $\begin{array}{l}\text { Most natural particle } \\
\text { emitters contain } \\
\text { impurity. }\end{array}$ & $\sqrt{ }$ & $\sqrt{ }$ & $\sqrt{ }$ & $\sqrt{ }$ & $\begin{array}{c}\mathrm{x} \\
\text { (except } \\
\mathrm{H}-\mathrm{O}) \\
\end{array}$ & $\sqrt{ }$ \\
\hline $\begin{array}{l}\text { Natural emitters are } \\
\text { most likely } \\
\text { conducting. }\end{array}$ & $\sqrt{ }$ & & $\sqrt{ }$ & $\sqrt{ }$ & $\mathrm{x}$ & $\sqrt{ }$ \\
\hline $\begin{array}{l}\text { Prefer protrusion } \\
\text { model. }\end{array}$ & $\mathrm{x}$ & & $\sqrt{ }$ & $\sqrt{ }$ & $\mathrm{x}$ & \\
\hline $\begin{array}{l}\text { Artificial insulating } \\
\text { particles do not emit } \\
\text { or emit weakly, while } \\
\text { conducting ones emit } \\
\text { strongly, in DC. }\end{array}$ & $\sqrt{ }$ & & $\sqrt{ }$ & & & \\
\hline $\begin{array}{l}\text { Artificial insulating } \\
\text { particles emit } \\
\text { strongly in RF. }\end{array}$ & & & $\begin{array}{c}\sqrt{ } \\
\text { (rf test) }\end{array}$ & & & \\
\hline $\begin{array}{l}\text { Anodization has no } \\
\text { consistent effect on } \\
\text { FE. }\end{array}$ & $\sqrt{ }$ & & $\sqrt{ }$ & $\sqrt{ }$ & & \\
\hline $\begin{array}{l}\text { Switching \& } \\
\text { multiswitching } \\
\text { (inference: } \\
\text { insulating \& } \\
\text { semiconducting } \\
\text { material likely } \\
\text { present) }\end{array}$ & $\sqrt{ }$ & & $\sqrt{ }$ & & & $\sqrt{ }$ \\
\hline
\end{tabular}


emission. The unavailability of the state-of-the-art cleaning techniques and cleanroom environment may be the reason for the disagreement between the University of Aston and the rest.

Field emission current instability has been observed in this work. Although sample desorption in vacuum helped some emitters to stabilize, for others the instability remained. The electric force from strong electric field may have dislodged emitters microscopically, causing an unstable electrical contact, hence an unstable current.

Switching and sometimes hysteresis in I-V characteristics were observed on some emitters in this work, but these emitters showed no significant difference in their appearances or composition from other stable emitters.

\subsubsection{The role of surface adsorbate in field emission}

Although the effect of surface adsorbate has been mentioned briefly in the first several chapters, it is a topic that needs to be addressed in more detail in order to explain the reason behind the thermal desorption performed on our samples before each field emission experiment and the theoretical grounds for the improbability of intrinsic emitters causing vacuum arcs at nA level. There is evidence to suggest that surface adsorbate on electrodes plays a role in real-life EFE. Among them is the phenomenon of current instability commonly found in field emitters, although according to F-N theory, EFE current from an ideal emitter should be stable and temperature-independent. The current instability can be significantly improved by current conditioning, i.e., maintaining a certain current, usually in the $\mu \mathrm{A}$ range, for a few minutes or longer. Increased gas evolution $\left(\mathrm{H}_{2}, \mathrm{CO} / \mathrm{N}_{2}, \mathrm{CO}_{2}\right.$ and water $)$ is observed during the conditioning. ${ }^{70}$ Thermal desorption on cathodes by heating also helps to improve current stability. ${ }^{47}$

Measurement during pre-breakdown and breakdown shows a characteristic increase in total pressure and the desorbed gas consists predominantly of $\mathrm{CO}$ and 
$\mathrm{CO}_{2}$ for all electrode materials, ${ }^{71-75}$ which can be explained by their high solubility in most metals, and the thermal oxidation of carbon, a common residual impurity in metals.

The stable current before pre-breakdown can be caused by EFE. Mesyats and his coworkers through both theoretical and experimental work proposed a three-stage breakdown model for electron emission initiated breakdown, ${ }^{76-87}$ which includes, firstly, the heating of the cathode emitter to reach thermal instability and the onset of an explosive electron emission, and secondly, the formation and expansion across the gap of microplasma created from the vaporized cathode emitter, and thirdly, the subsequent formation of anode microplasma that crosses the gap in reverse direction and helps to strike an arc across the gap. In this work the emission current is controlled to below several $\mathrm{nA}$, which is very unlikely to cause intrinsic emitters to reach their melting point by Joule heating, because the good thermal contact prevents the intrinsic emitters from reaching temperatures much higher than that of the bulk material. Even for a loosely-attached particle of $20 \mu \mathrm{m}$ size, the minimum power needed for the particle to reach a melting point of $1800 \mathrm{~K}(\mathrm{Fe})$ or $2700 \mathrm{~K}(\mathrm{Nb})$ is on the order of $10-15 \mathrm{~mW}(\mathrm{Fe})$ and $15-20 \mathrm{~mW}(\mathrm{Nb})$, as measured by Tan at Saclay ${ }^{47}$ which can not be provided by Joule heating from FE or RF heating, or the Nottingham effect. One plausible explanation for this discrepancy is that a large burst of desorbed gas plays an important role in helping the particle to reach thermal instability.

As a result, samples to be studied need to be outgassed not only to obtain a stable current measurement but also to lower the chance of vacuum arc. We use a $300 \mathrm{~W}$ Halogen lamp placed at one focal point of an ellipsoidal reflector to focus light onto the sample surface. The lamp and reflector are placed outside a viewport on the HT chamber to heat the sample to above $200^{\circ} \mathrm{C}$ for $\sim 6$ hours before the sample is transferred to the SFEM chamber for FE study. Vacuum arc, however, still can not be completely prevented due to its complex nature. As a result, steps need to be 
taken to reduce the damage induced by a vacuum arc. In this work, we used a vacuum-compatible current limiting resistor of 100 G $\Omega$ placed inside the vacuum chamber to reduce the energy released in the event of gap breakdown.

\subsection{Possible experiments and potential application for the apparatus}

Field emission will understandably still be a major obstacle in reaching $100 \mathrm{MV} / \mathrm{m}$ peak fields in multicell cavities. Therefore, the SFEM system can be used to optimize the variables for new as well as existing surface treatment techniques, $\mathrm{BCP}$, electropolishing and other possible new techniques, and to evaluate handling procedures and storage methods before being applied to $\mathrm{Nb}$ cavities or other devices. Field emission studies on samples subject to the same deep drawing as cavities would provide guidance on the minimal surface removal needed to achieve desirable field emission performance. Other processes that are being developed and intended for SRF application or DC high voltage vacuum insulation, for instance, laser or electron beam processed material, can also be evaluated by the apparatus for their FE property. Other potential uses for the system include examination of material relevant to field emission displays.

Field emission characterization of a GaAs photocathode for use on the Jefferson Lab FEL injector was performed. Operating experience with the photocathode showed that after a certain period of operation, field emission from the GaAs wafer can limit extracting electric field for photoelectrons to $\sim 10 \mathrm{MV} / \mathrm{m}$, which in turn limits the available current. The field emission scan on a new wafer showed that its initial condition is free of emission up to $60 \mathrm{MV} / \mathrm{m}$, except for three detected emitters that are all located near the samples' edge. Two emitters contain indium,

supposedly introduced by wafer handling tweezers. The scan and SEM picture of emitters are shown in Appendix B. A future experiment with a failed photocathode will help to shed light on the cause of the failure. Adjacent stainless steel electrodes 
in the injector might also have contributed to the field emission problem, and can be examined by the apparatus as well. It has been observed that ion-implanted stainless steel exhibits reduced field emission, which can be tested by the apparatus. Similar issues exist on the electrodes for the CEBAF injector.

\subsection{Summary}

The SFEM apparatus has been used to conduct over 50 FE scans (total scan area: $\sim 250 \mathrm{~cm}^{2}$ ) and performed microscopy characterizations at identified emitting sites. The system met design goals and has proven to work reliably and consistently.

Compared to other DC apparatus built elsewhere and described in this thesis, this system has advantages or improvements in:

- Low cost and operational flexibility

- Considerably larger scan area per sample, i.e., $4.9 \mathrm{~cm}^{2}$, compared to $1.44 \mathrm{~cm}^{2}$ from the University of Geneva or $\leq 1.77 \mathrm{~cm}^{2}$ from the University of Wuppertal. Larger samples are assumed to be better at representing extended surfaces such as in cavities. Larger samples also translate to a better work efficiency and a larger total scan area due to reduced work overhead, i.e., more reliable statistics with respect to emitter characteristics and emission density. Of course, it is also much more difficult to achieve emission free performance from a larger sample.

- Patent-pending sample and sample holder design enables us to experimentally unambiguously distinguish external particles from intrinsic impurities. Microscopic appearance in SEM alone can be misleading due to the large depth of field.

- Higher resolution in situ SEM than other DC devices.

The system also has disadvantages:

- Accuracy in re-locating emitters is $\pm 100-200 \mu \mathrm{m}$, not as good as those combining SEM with FE scanning in the same chamber. 
- EDS is not as surface sensitive as Auger Electron Spectrometry.

Field emission sites on a number of $\mathrm{Nb}$ samples were located and characterized, after being prepared by BCP chemical etching or electropolishing, rinsed with high pressure ultra pure water or in ultrasonic rinse.

The findings and contribution of this thesis work are:

- The first, to our knowledge, comparative field emission studies on BCP chemical etched and electropolished $\mathrm{Nb}$ samples at high fields. No inherent difference in emitter nature, or significant difference in emission density is observed up to 140 $\mathrm{MV} / \mathrm{m}$, which refutes the frequently quoted arguments that smoother surface is preferred for suppressing FE due to the reduced geometrical enhancement factor. In light of this finding, mechanical polishing is deemed unnecessary, or even harmful because of the added risk of deep contamination.

- No evidence of material bulk impurity as emitters is observed up to $140 \mathrm{MV} / \mathrm{m}$, for $\mathrm{BCP}$ or electropolished $\mathrm{Nb}$, which is also somewhat in disagreement with Wuppertal's findings shown in Figure 3.3. However, all of their localized emitters are particles containing $\mathrm{Nb}$ and $\mathrm{Fe}$, consistent with the majority of the emitters from our samples, which were later removed by improving the machining process and avoiding scanning the contaminated edge area, thus their identified emitters may not be bulk impurities. Nonetheless, the trend of emitter density increasing with material impurity grade may still be true, only that at RRR 300, the density of impurity conglomerate is low enough to approach $0 /\left(18 \times 4.9 \mathrm{~cm}^{2}\right) \approx 0 /\left(88 \mathrm{~cm}^{2}\right)(18$ samples from Table 5.2, 5.3, 6.1 and Figure 7.3), or the impurity will only become emitters at higher fields.

- All identified emitters are foreign microparticles. There are arcs during field emission scan, which are likely caused by loosely-attached particles as well. The emitter plus vacuum arc density is the lowest ever achieved on $\mathrm{Nb}$ samples and

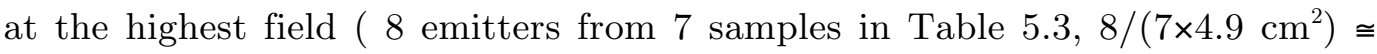
$\left.0.23 / \mathrm{cm}^{2}\right)$. And this is achieved by BCP and ultrasonic rinse only and without 
heat treatment or high pressure rinse. The statistical comparison with other institutions is shown in Figure 9.1. Further reduction would require a cleaner environment, e.g., Class 100 or 10 cleanroom for particle control. Emission free samples up to $100 \mathrm{MV} / \mathrm{m}$ were achieved by BCP (\#75-2) and EP (\#65-4) with ultrasonic rinse. Emission free up to $140 \mathrm{MV} / \mathrm{m}$ was achieved by $\mathrm{BCP}$ and ultrasonic rinse (sample \#81-1) and by BCP and HPWR (sample \#71-1).

In the Cornell rf studies, some emitters that appeared at lower field may get processed away as the field is raised, while others remained as emitters with the current increasing exponentially with field. In addition more new emitters appear and the emitter density also roughly increases exponentially with field (refer to Figure 9.1). DC studies by Geneva showed similar trends. Therefore not only does

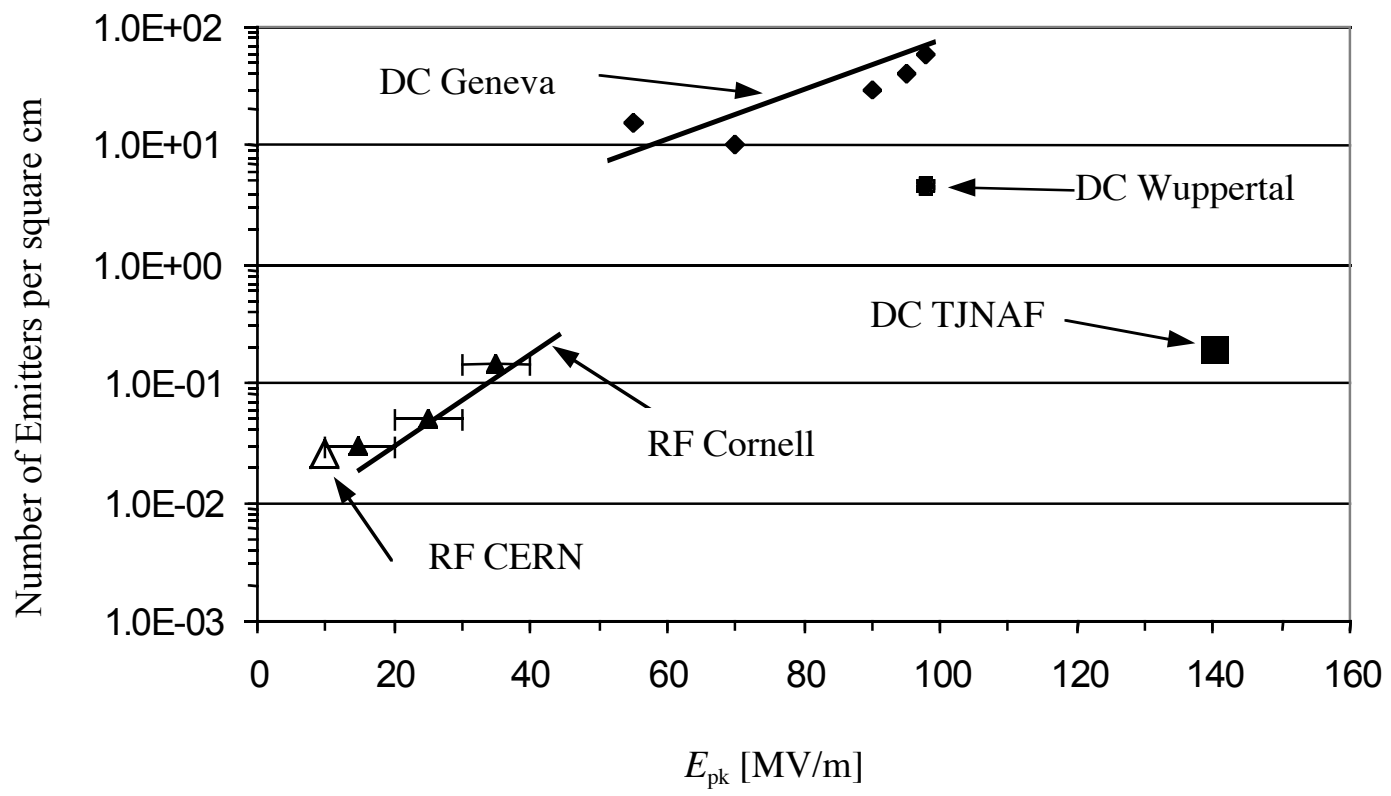

Figure 9.1: Density of field emission sites identified in DC (Geneva and Wuppertal) and RF studies (CERN and Cornell) ${ }^{1}$ (i.e., density of emitters with $E_{\text {onset }} \leq E_{\mathrm{pk}}$ identified in a DC scan or an RF operation at $E_{\mathrm{pk}}$ surface field versus $E_{\mathrm{pk}}$ ), with the addition of this work (TJNAF). 
the field emission current increase exponentially with field, but also the emitter density. We can infer that FE will still be a major obstacle for reaching $100 \mathrm{MV} / \mathrm{m}$, especially in multicell cavities because of the enormous surface area of $\sim \mathrm{m}^{2}$. The Wuppertal samples showed a reduced density compared to Geneva samples because their samples were prepared in a Class 100 cleanroom while Geneva samples were exposed to ordinary room air. Nevertheless Wuppertal samples still showed a significant emitter density of $\sim 8$ emitters $/ \mathrm{cm}^{2}$. This thesis work has achieved a new low in emitter density, which suggests future directions or emphasis for reducing FE in cavities.

- Stringent control of the machining process to minimize the chances of embedding contaminant in the surface has proven to be critical in achieving the low emitter density in this work. This process has not been given due attention and study in the past. With inconsistent machining, a set amount of $\mathrm{BCP}$ removal will not produce consistent FE performance, and high pressure water rinse or ultrasonic rinse can only bring a certain level of improvement.

- The above conclusions should be applicable to other high purity materials used in high voltage vacuum gap or other high field devices in general.

- Extended natural drying process in a class 10 cleanroom was compared to methanol rinse followed by laminar drying on $\mathrm{Nb}$ samples. No significant difference was observed in emitter density up to $140 \mathrm{MV} / \mathrm{m}$, hence there is no evidence of new emitters created by water reaction.

- Field emission was tested on a preliminary $\mathrm{Nb}$ thin film sample energetically deposited on $\mathrm{Cu}$ substrate. No evidence of intrinsic emitters was found on the film; however, the $\mathrm{Cu}$ substrate finish needs to be improved to obtain a more definitive conclusion. 


\section{Appendix A}

\section{Illustration of LabView control panel and block diagram for field emission scan}
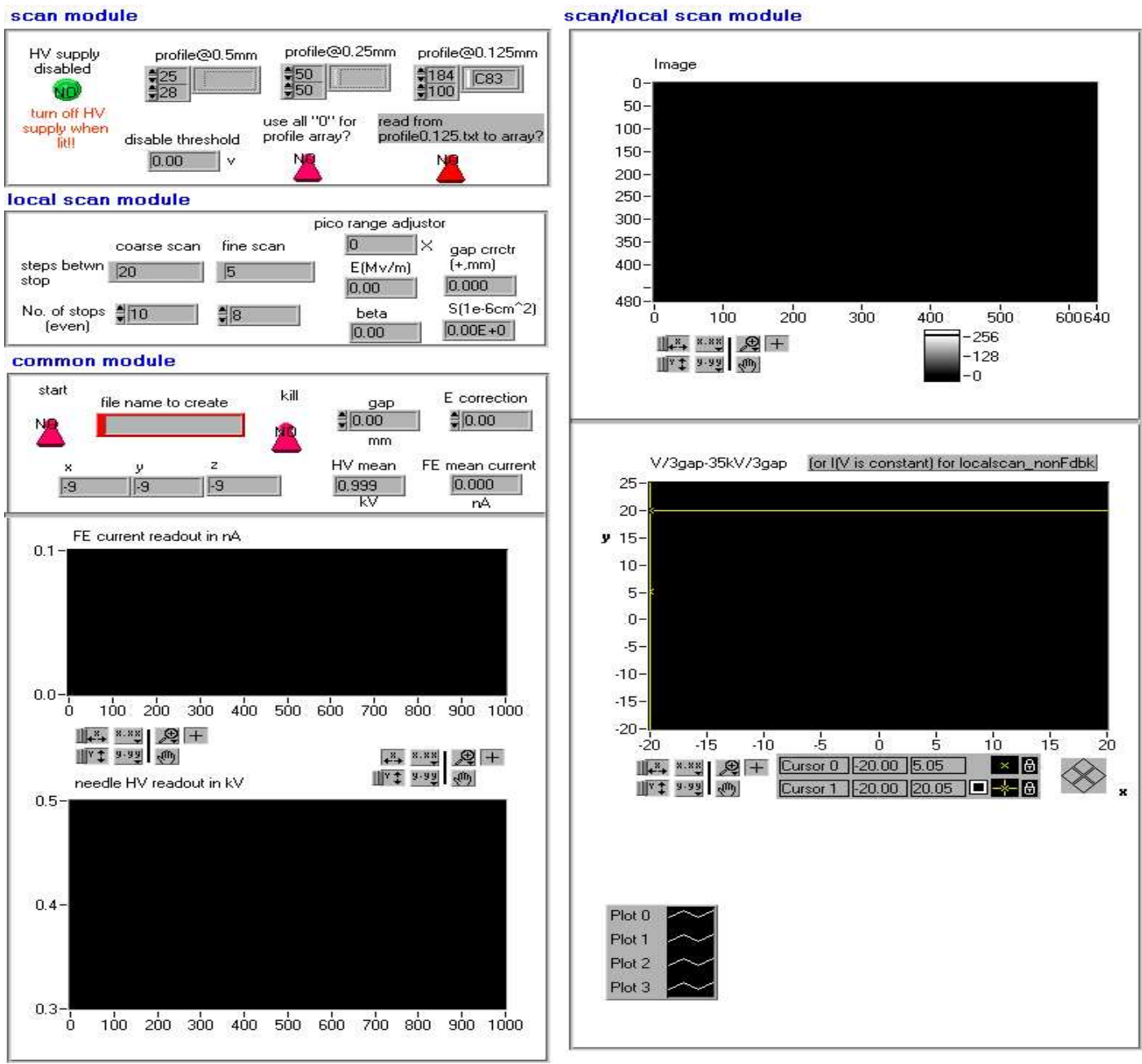


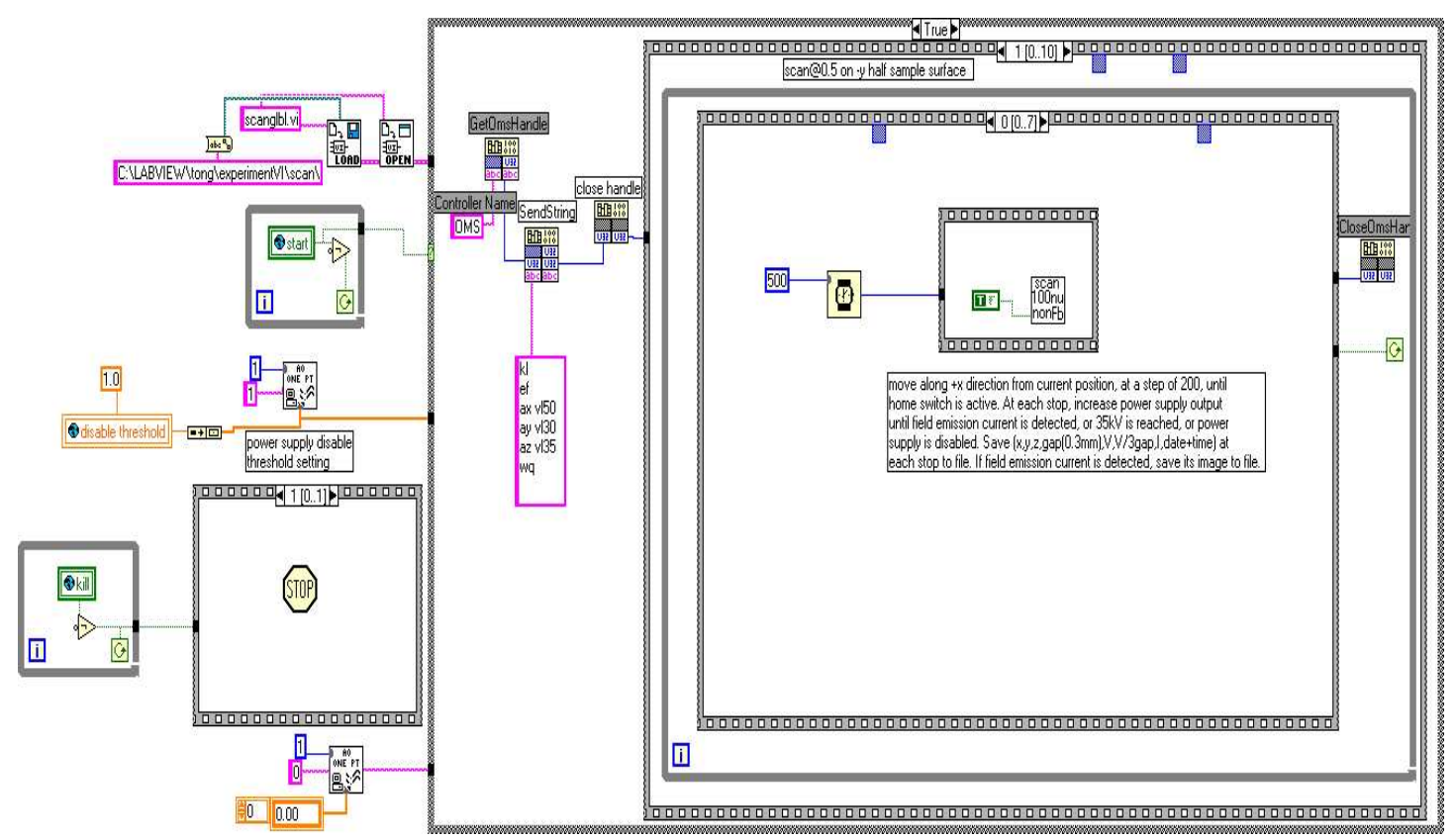




\section{Appendix B}

\section{Illustration of GaAs photocathode field emission scan and identified emitters}

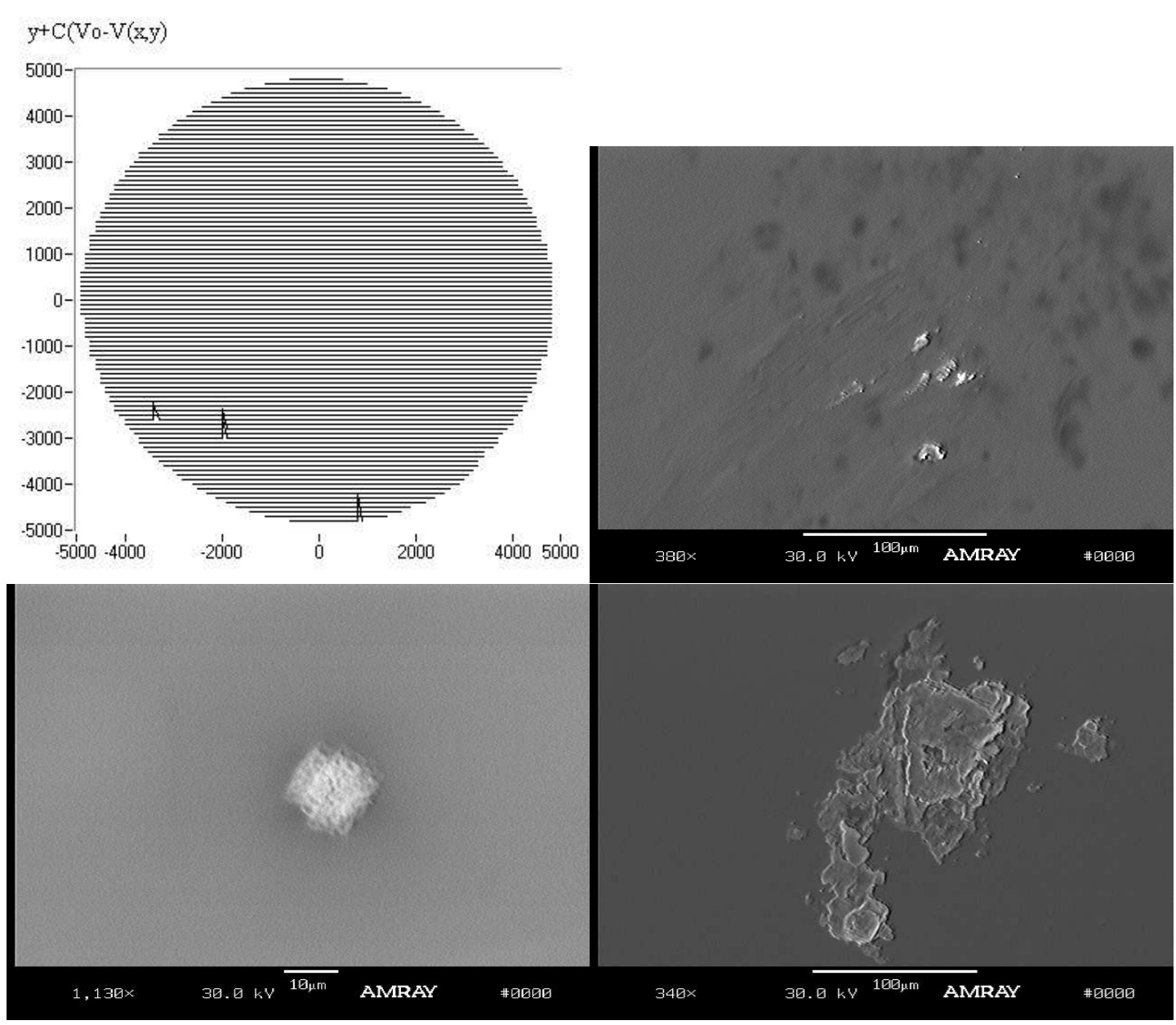

Scanning field $=60 \mathrm{MV} / \mathrm{m}$. Two emitters contain indium (contaminant from wafer handling tweezers), the other one has Ga and As only (picture at lower left). 


\section{Bibliography}

${ }^{1}$ H. Padamsee, J. Knobloch, and T. Hays, RF Superconductivity for Accelerators (Wiley and Sons, New York, 1998).

2 J. Matricon and D. Saint-James, Superheating fields in superconductors, Physics Letters 24 A, 241-242 (1967).

${ }^{3}$ H. Piel, Superconducting cavities, CERN Accelerator School: Superconducting in Particle Accelerators, Hamburg, Germany, 149-196 (1988). CERN publication 8904.

${ }^{4}$ Proc. of the 7th Workshop on RF Superconductivity, CE Saclay, Gif sur Yvette, France, (1995), edited by B. Bonin.

${ }^{5}$ Proc. of the 8th Workshop on RF Superconductivity, Padova, Italy, (1997).

${ }^{6}$ R. H. Fowler and L. Nordheim, Proc. Roy. Soc. London A 119, 173 (1928).

7 L. Nordheim, Proc. Roy. Soc. London A 121, 626 (1928).

8 W. Schottky, Z. Physik 14, 63 (1923).

${ }^{9}$ C. B. Duke, Tunneling in Solids (Academic Press, New York, 1969).

${ }^{10}$ R. H. Good and E. W. Müller, Handbuch der Physik, (Springer-Verlag, Berlin, 1956).

${ }^{11}$ C. A. Spindt, I. Brodie, L. Humphrey, and E. R. Westerberg, J. Appl. Phys. 47, $5248(1976)$.

${ }^{12} \mathrm{Ph}$. Niedermann, Ph. D thesis, University of Geneva, 1986.

${ }^{13}$ N. Pupeter, A. Göhl, T. Habermann, A. Kirschner, E. Mahner, G. Müller, and H. Piel, Influence of surface roughness and preparation, bulk purity and heat 
treatment on electron field emission from $\mathrm{Nb}$ and $\mathrm{Nb}_{3} \mathrm{Sn}$, Proc. of the $7^{\text {th }}$ Workshop on RF Superconductivity, Gif-sur-Yvette, France, 67-77, 1995.

${ }^{14}$ R. J. Noer, Appl. Phys. A 28, 1 (1982).

${ }^{15}$ R. V. Latham, High Voltage Vacuum Insulation (Academic Press, London, 1981).

${ }^{16}$ P. A. Chatterton, Proc. II-DEIV, 195-206 (1966).

${ }^{17}$ H. P. S. Powell and P. A. Chatterton, Vacuum, 20, 419-29 (1971).

${ }^{18}$ R. Hackam, Proc. IEE, 119, 377-84 (1972).

${ }^{19}$ R. E. Hurley, J. Phys. D: Appl. Phys. 12, 2229-45 (1979).

${ }^{20}$ R. E. Hurley and P. J. Dooley, J. Phys. D: Appl. Phys. 10, L195-201 (1977).

${ }^{21}$ N. K. Allen and R. V. Latham, Proc. VIII-DEIV, c6 1-9 (1978).

${ }^{22}$ F. Rohrbach, CERN Report, 71-5/TC-L (1971).

${ }^{23}$ G. E. Vibrans, Tech. Report 353, Lincoln Lab., MIT (NTIS ESDTDR 64-327) (1964).

${ }^{24}$ R. P. Little, and S. T. Smith, J. Appl. Phys. 36, 1502 (1965).

${ }^{25}$ M. Jimenez, R. J. Noer, G. Jouve, J. Jodet, and B. Bonin, J. Phys. D: Appl. Phys. 27, 1038-1045 (1994).

${ }^{26}$ M. Jimenez, R. J. Noer, G. Jouve, C. Antoine, J. Jodet, and B. Bonin, J. Phys. D: Appl. Phys. 26, 1503-1509 (1993).

${ }^{27}$ T. Habermann, A. Göhl, G. Müller, N. Pupeter, H. Piel, Proc. of the 5th Eur. Particle Acc. Conf., Barcelona, Spain, 2082-2084 (1996).

${ }^{28}$ C. S. Athwal, and R. V. Latham, Physica 104C, 46-49 (1981).

${ }^{29}$ C. S. Athwal, K. H. Bayliss, et al. IEEE Trans. Plasma Science, Vol. PS-13, No.5, 226-229 (1985).

${ }^{30}$ N. K. Allen and R. V. Latham, Proc. VIII-DEIV, B3, 1-13 (1978).

${ }^{31}$ N. K. Allen, B. M. Cox and R. V. Latham, J. Phys. D: Appl. Phys. 12, 969-78 (1979).

${ }^{32}$ C. S. Athwal, R. V. Latham, J. Phys. D: Appl. Phys. 17, 1029 (1984).

${ }^{33}$ R. V. Latham, Vacuum 32, 137, (1982). 
${ }^{34}$ C. S. Athwal and R. V. Latham, Physica C 104, 189, (1981).

${ }^{35}$ N. S. Xu. High voltage vacuum insulation, edited by R. V. Latham (Academic Press, London, 1995).

${ }^{36}$ R. E. Hurley, J. Phys. D: Appl. Phys. 12, 2247-52 (1979).

${ }^{37}$ G. Dearnaley, A. M. Stoneham and D. V. Morgan, Rep. Prog. Phys. 33, 1129-91 (1970).

${ }^{38}$ K. W. Boer, and S. R. Ovshinsky, J. Appl. Phys. 41, 2675-81 (1970).

${ }^{39}$ C. B. Duke and M. E. Alferieff, J. Chem. Phys. 46, 923-37 (1967).

${ }^{40}$ J. W. Gadzuk, Phys. Rev. 81, 2110-29 (1970).

${ }^{41}$ R. V. Latham, IEEE Trans. Electrical Insul. EI-18, 194 (1983).

${ }^{42}$ P. H. Cutler, J. He, et al. J. Vac. Sci. Technol. B11(2), 387-391 (1993).

${ }^{43} \mathrm{~W}$. Weingarten, Proc. of the $2^{\text {nd }}$ workshop on RF superconductivity, CERN, Geneva, 551, 1984, edited by H. Lengeler.

${ }^{44}$ H. Hübner, Optik 64, 113 (1983); H. Hübner, J. de Physique Colloque suppl. 45, C9-279 (1984).

${ }^{45} \mathrm{Ph}$. Niedermann, N. Sankarraman, O. Fisher, Proc. of the $2^{\text {nd }}$ Workshop on RF Superconductivity, 583 (1984).

${ }^{46}$ E. Mahner, G. Müller, H. Piel, and N. Pupeter, J. Vac. Sci. Technol. B 13 (2), 607 (1995).

${ }^{47}$ J. Tan, Proc. of the 7th Workshop on RF Superconductivity, CEA/Saclay, 105 (1995), edited by B. Bonin.

${ }^{48}$ D. Moffat, P. Barnes, T. Flynn, J. Graber, L. Hand, W. Hartung, T. Hayes, J. Kirchgessner, J. Knobloch, R. Noer, H. Padamsee, D. Rubin, and J. Sears, Proc. of the 5th Workshop on RF Superconductivity, DESY, 245 (1991).

${ }^{49}$ A. Curtoni Chianelli, A. Zeitoun-Fakiris, J. Jodet, I. Regardin, Proc. of the $5^{\text {th }}$ Workshop on RF Superconductivity, 700 (1991).

${ }^{50}$ B. Bonin, Proc. of the 6th workshop on RF superconductivity, 1033 (1993). 
${ }^{51}$ E. Mahner, R. Heiderhoff, N. Minatti, G. Müller, H. Piel, N. Pupeter, Proc. of the 5th workshop on RF superconductivity, 694 (1991).

${ }^{52}$ E. Mahner, N. Minatti, H. Piel and N. Pupeter, Applied Surface Science, 67, 23-28 (1993).

${ }^{53}$ E. Mahner, Proc. of the 6th workshop on RF superconductivity, 252 (1993).

${ }^{54}$ T. W. Haas, J. T. Grant, G. J. Dooley, J. Vac. Sci. Technol., 7, 43 (1969).

${ }^{55}$ L. A. Harris, J. Appl. Phys., 39, 1428 (1968).

${ }^{56}$ S. Hofmann, Vacuum, 40, 9 (1990).

${ }^{57}$ S. Hofmann, Materials Science Engineering, 42, 55 (1980).

${ }^{58}$ S. Hofmann, G. Blank, H. Schultz, Z. Metallkde, 67, 189 (1976).

${ }^{59}$ B. M. Cox, J. Phys. D: Appl. Phys. 8, 2065 (1975).

${ }^{60}$ R. Sundelin, E. von Borstel, J. Kirchgessner, D. Rice, and M. Tigner, IEEE Transactions on Nuclear Science, \#3, Vol. NS-20, 98(1973).

${ }^{61}$ W.J. McTegart, R. G. Vines, Trans. Aust. Inst. Metals, 5, 107 (1952).

${ }^{62}$ K. L. Mittal, Treatise on Clean Surface Technology, edited by K. L.Mittal (Plenum Press, New York and London, 1987).

${ }^{63}$ E. Kako, S. Noguchi, M. Ono, K. Saito, T. Shishido, H. Safa, J. Knobloch, and L. Lilje, Proc. of the $9^{\text {th }}$ Workshop on RF Superconductivity (1999).

${ }^{64}$ L. Lilje, Proc. of the $10^{\text {th }}$ workshop on RF Superconductivity, Japan, (2001), to be published.

${ }^{65}$ T.Lyman, Metals Handbook, edited by T. Lyman, Vol. 8, Metallography, structure and phase diagram, (American Society for Metals, $8^{\text {th }}$ edition, 1973).

${ }^{66}$ H. Diepers, O. Schmidt, H. Martens, F. S. Sun, Phys. Letter. 37A. 139 (1971).

${ }^{67}$ Gmelin Handbuch der Anoganischen Chemie, Band 49 NIOB .

${ }^{68}$ J. Pagetti and J. Talbot, Corrosion-Traitments-Protection-Finition 15, 171 (1967).

${ }^{69}$ G. Wu, Ph. D thesis, Virginia Polytechnic Institute and State University, 2002. 
${ }^{70}$ V. A. Nevrovski, V. I. Rakhovski, V. G. Zhurbenko, V. A. Zernaev, J. Phys. D.: Appl. Phys. 14, 215 (1981).

${ }^{71}$ J. C. L. Cornish, R. V. Latham and E. Braun, Proc. IV-DEIV, 28-32 (1970).

${ }^{72}$ B. Jüttner, Exper. Tech. Phys. 19, 45-48 (1970).

${ }^{73}$ A. Ghani, Ph. D. Thesis, University of Aston, U.K. (1971).

${ }^{74}$ J. A. Panitz, J. Appl. Phys. 44, 372-5 (1973).

${ }^{75}$ J. A. Panitz, J. Appl. Phys. 45, 1112-4 (1974).

${ }^{76}$ G. K. Kartsev, G. A. Mesyats, D. I. Proskurovsky, V. P. Rotshteyn and G. N. Fursey, Sov. Phys.-Dokl., 15, 475-7 (1970).

${ }^{77}$ G. A. Mesyats and D. I. Proskurovsky, Sov. Phys. J., 11, 49-51 (1968).

${ }^{78}$ S. P. Bugaev, G. A. Mesyats and D. I. Proskurovsky, Sov. Phys. -Dokl, 14, 605-6 (1969).

${ }^{79}$ S. P. Bugaev, V. I. Koshelev and M. N. Timoleev, Sov. Phys. J., 17, 193-196 (1974).

${ }^{80}$ R. B. Baksht, V. A. Kokshenov and V. I. Manylov, Sov. Phys: Tech. Phys., 20, 1069-71 (1975).

${ }^{81}$ S. P. Bugaev, E. A. Litvinov, V. V. Lopatin and G. A. Mesyats, Nanosecond High Power Electron Pulses, Nauka, Novosibirsk, 52 (1974).

${ }^{82}$ G. A. Mesyats, Zhurn, Tekhn. Fiz., 44, 1521-4 (1974).

${ }^{83}$ D. I. Proskurovsky and Ya. Ya. Urike, Proc. IV-DEIV, 78-81 (1970).

${ }^{84}$ E. A. Litvinov, G. A. Mesyats, A. F. Shubin, L. M. Buskin and G. N. Fursey, Proc. VI-DEIV, 107-111 (1974).

${ }^{85}$ E. A. Litvinov, G. A. Mesyats, D. I. Proskurovsky and E. G. Yankelevitch, Proc. VII-DEIV, 55-67 (1976).

${ }^{86}$ E. A. Litvinov, G. A. Mesyats and A. F. Shubin, Sov. Phys. J., 13, 537-40 (1970).

${ }^{87}$ S. P. Bugaev, E. A. Litvinov, G. A. Mesyats and D. I. Proskurovsky, Sov. Phys. Usp., 18, 51-61 (1975). 


\section{VITA}

Tong Wang was born in September 1970 in Heilongjiang Province, China. She earned her Bachelor of Science and Master of Science in Physics from Beijing University in July 1992 and 1995 respectively. In Septermber 2002, she received her Ph.D. in Physics from Virginia Polytechnic Institute and State University in Blacksburg, Virginia.

Tong married her husband Xiaodong (Sheldon) in May 1995. 